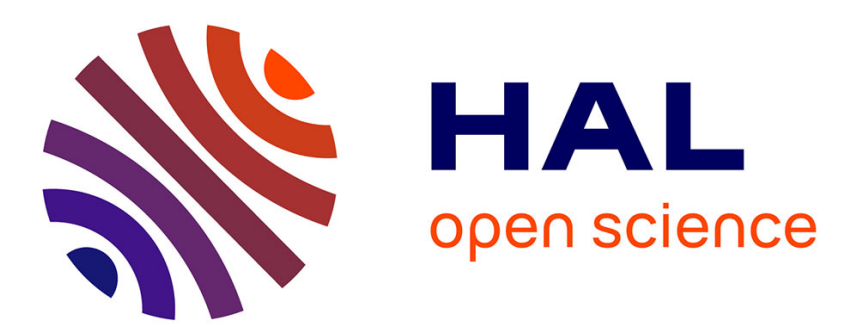

\title{
Analysis of an observer strategy for initial state reconstruction of wave-like systems in unbounded domains
}

\author{
Sébastien Imperiale, Philippe Moireau, A Tonnoir
}

\section{To cite this version:}

Sébastien Imperiale, Philippe Moireau, A Tonnoir. Analysis of an observer strategy for initial state reconstruction of wave-like systems in unbounded domains. ESAIM: Control, Optimisation and Calculus of Variations, 2019, 26 (45), 10.1051/cocv/2019026 . hal-01898440v3

HAL Id: hal-01898440

https://hal.inria.fr/hal-01898440v3

Submitted on 9 Jul 2019

HAL is a multi-disciplinary open access archive for the deposit and dissemination of scientific research documents, whether they are published or not. The documents may come from teaching and research institutions in France or abroad, or from public or private research centers.
L'archive ouverte pluridisciplinaire HAL, est destinée au dépôt et à la diffusion de documents scientifiques de niveau recherche, publiés ou non, émanant des établissements d'enseignement et de recherche français ou étrangers, des laboratoires publics ou privés. 


\title{
ANALYSIS OF AN OBSERVER STRATEGY FOR INITIAL STATE RECONSTRUCTION OF WAVE-LIKE SYSTEMS IN UNBOUNDED DOMAINS
}

\author{
S. Imperiale ${ }^{1}$, P. MoIreau ${ }^{1}$ And A. TONNOIR ${ }^{1,2}$
}

\begin{abstract}
We are interested in reconstructing the initial condition of a wave equation in an unbounded domain configuration from measurements available in time on a subdomain. To solve this problem, we adopt an iterative strategy of reconstruction based on observers and time reversal adjoint formulations. We prove the convergence of our reconstruction algorithm with perfect measurements and its robustness to noise. Moreover, we develop a complete strategy to practically solve this problem on a bounded domain using artificial transparent boundary conditions to account for the exterior domain. Our work then demonstrates that the consistency error introduced by the use of approximate transparent boundary conditions is compensated by the stabilization properties obtained from the use of the available measurements, hence allowing to still be able to reconstruct the unknown initial condition.
\end{abstract}

Résumé. Nous nous intéressons au problème de recontruction de la donnée initiale pour une équation d'ondes posée en domaine non borné à l'aide de mesures en temps sur un sous domaine. Pour résoudre ce problème, nous utilisons une approche itérative de reconstruction basée sur une formulation type observateurs et retournement temporel. On prouve la convergence de l'algorithme de reconstruction dans le cas de mesures non bruitées et sa stabilité par rapport à des mesures bruitées. De plus, nous développons une méthodologie complète pour résoudre numériquement le problème en domaine borné en utilisant des conditions transparentes (absorbantes) sur les frontières artificielles bornant le domaine. Nous montrons en particulier que l'erreur de consistance introduite par les conditions absorbantes (non exactes) est compensée par les propriétés de stabilisation obtenues grâce aux mesures, ce qui permet d'être toujours en mesure de reconstituer la donnée initiale.

1991 Mathematics Subject Classification. 65M32, 93B07, 93D20, 35L05.

The dates will be set by the publisher.

\section{CONTENTS}

1. Introduction

2. Problem formulation and main results

2.1. Problem settings

2.2. An iterative reconstruction strategy

3. Convergence analysis

3.1. The reconstruction-error operator

3.2. Noisy data analysis

3.3. The observability inequality

Keywords and phrases: wave equation in unbounded domain, back and forth observer, state estimation, data assimilation ${ }^{1}$ Inria - LMS, Ecole Polytechnique, CNRS, Université Paris-Saclay.

Inria Saclay-Ile-de-France, 1 rue Honoré d'Estienne d'Orves, 91120 Palaiseau France, e-mail: philippe.moireau@inria.fr

${ }^{2}$ Normandie Université, INSA de Rouen Normandie, LMI (EA 3226 - FR CNRS 3335), 76000 Rouen, France, 685 Avenue de l'Université, 76801 St Etienne du Rouvray, Cedex France. 
4. Reformulation in bounded domain

4.1. Example of a bounded domain reformulation in 1D 16

4.2. An abstract framework $\quad 17$

4.3. The abstract back-and-forth algorithm 18

4.4. Stability analysis 22

5. Application to the wave equation with approximate transparent boundary conditions 23

5.1. Specific formulation in the abstract framework 24

5.2. Application of the abstract back-and-forth algorithm 26

6. Numerical investigations 30

6.1. The 1D case 30

6.2. 2D examples 36

Appendix A. An energy equirepartition result $\quad 43$

Appendix B. Proof of Lemma $5.1 \quad 44$

Appendix C. Proof of an observation inequality by multipliers 44

References $\quad 48$

\section{INTRODUCTION}

This work is motivated by the development of the transient elastography technique [8]. This technique consists in imaging the propagation (in time) of ultrasonic waves inside a biological tissue in order to deduce its physical properties, these properties being of major importance for medical applications $[17,18,48,51]$. A typical illustration of this technique is given in Figure 1 on an elastic phantom, namely in a noise limited configuration. In this case, the ultimate objective is to characterize an inclusion.

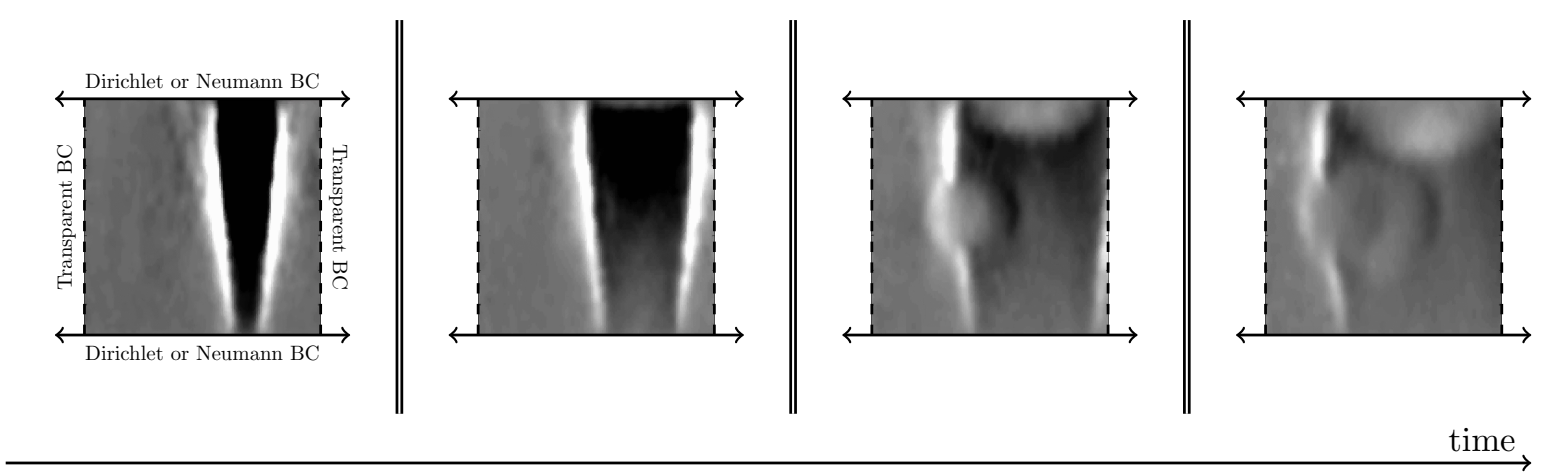

FiguRE 1. Propagation of a shear wave in an elastic phantom containing a harder inclusion.

The domain is bounded vertically and unbounded horizontally. In the spirit of [8, Figure 9]

- Courtesy M. Pernot (Institut Langevin).

Inversion of elastography measurements has been widely studied in the literature - see for instance $[1,3,40]$ among many others - with methods adapted to the context of application of the technique, namely harmonic configurations or transient configuration as we consider here. In the transient case, our motivation is to ultimately address this problem as an inverse problem of state and parameter estimation given - possibly partial - volume data in time for a time-domain wave equation. Moreover, we expect to address this question from an optimal control point of view. Therefore in this work and as a first step, we focus on some of the specific difficulties arising when formulating such an optimal control estimation problem on time evolution wave problems set on unbounded domains.

A first strategy, with an optimal control point of view [38] for estimation purposes [6], consists in minimizing a least square functional involving the discrepancy between the measurements and the data generated by a model - see for instance [6] on this principle applied to general evolution equations or [1] when considering harmonic wave formulations in the elastography context. One drawback of this approach can be the number 
of corresponding iterations as it is typically the case when using first order gradient methods for minimizing the functional.

A second strategy consists in using a sequential estimation approach as originally envisioned in optimal filtering estimation approaches [6]. A major advantage of this approach is that a complete estimation is performed in only one iteration of the direct estimator - with eventually one iteration of the adjoint estimator when also reconstructing the initial condition. The drawback yet is that this method is not practically tractable when applied to partial differential equations since it imposes to solve a Riccati operator equation for computing the observer feedback.

In the context of wave equations, however, it has been proposed to define a so-called Back-and-Forth Luenberger observer [49] as a suboptimal estimator - in the sense of relaxing the optimal least square criterion - that allows a faster reconstruction than a classical gradient based functional minimization at a reasonable computational cost of the feedback. This tractable state feedback is then compatible with an additional parameter feedback [43] when jointly estimating the state and the parameters of the model. Note incidentally that, the state estimation feedback is, in fact, a mandatory estimation component even when only focusing on parameters identification problems for wave equations. Indeed, the conservative property of the wave system tends to accumulate all the error sources during the estimation procedure [43].

Our objective in this paper is to extend such a Back-and-Forth Luenberger strategy in a context inspired from transient elastography problems, namely when the system of interest must be considered as unbounded in at least one direction. As the parameter identification procedure proposed in [43] is not impacted by the unboundedness of the underlying problem, we focus on this article on the remaining difficulty which is to adapt the algorithm proposed in [49] to unbounded domain configurations.

To this end, we propose several contributions. The first contribution is theoretical and consists in setting the necessary assumptions under which the observability condition underlying the convergence of the algorithm proposed by [49] can be extended to unbounded domain configurations. In [49], the observability condition is directly related to the Geometric Control Condition. In our case, we will need a condition ensuring that all the information contained in the initial condition is observed. Therefore, only an initial condition of support surrounded by the observation domain can be reconstructed. Here, typically, we improve a similar result obtained by [30], where the GCC is used on rays that do not meet the initial condition support. The second contribution deals with the practical use of the reconstruction algorithm for unbounded domain. Indeed, solving efficiently direct - hence inverse - problems involving unbounded domain requires to define artificial boundary conditions - ideally transparent boundary conditions. Therefore, we propose a reconstruction strategy compatible with these boundary conditions, even if they are only approximate transparent boundary conditions, as soon as they are at least absorbing boundary conditions. Moreover, we demonstrate an original benefit of observer as we prove that the use of the available measurements compensate - or at least mitigate - the consistency error introduced by the approximated boundary conditions.

The outline of this paper is as follows. In Section 2, we present the problem formulation and recall the iterative back-and-forth algorithm. We then formulate our main result about the algorithm convergence in an unbounded domain case and its robustness with respect to noisy data. Then, in Section 3, we prove this result by relying on a specific Geometrical Control Condition on the observation region. We then show in Section 4 the consequences on our result of bounding the domain (using appropriate transparent boundary condition) with a motivation of practical resolution of our estimation problem. The abstract formalism presented in Section 4 is completed in Section 5 when considering specific choices of transparent boundary conditions. To conclude, in Section 6, we illustrate our approach by numerical investigations showing the importance of a proper discretization to ensure that the convergence properties obtained at the continuous level are kept at the discrete one. 


\section{Problem formulation AND MAin RESUlts}

\subsection{Problem settings}

We consider the following inverse problem: Find the initial field $u_{0}$ and corresponding velocity $u_{1}$ of the wave equation problem:

$$
\mid \begin{array}{lrr}
\partial_{t}^{2} u-\Delta u=0 & \text { in } & {[0, T] \times \mathcal{D},} \\
u=0 & \text { on } & {[0, T] \times \partial \mathcal{D},} \\
\left(u, \partial_{t} u\right)_{t=0}=\left(u_{0}, u_{1}\right) & \text { in } & \mathcal{D},
\end{array}
$$

given the measurement of the velocity $\partial_{t} u$ over time $[0, T]$ in a subdomain $\mathcal{D}_{\text {obs }} \subset \mathcal{D}$. To allow the reconstruction of the initial condition we will show that the observation domain $\mathcal{D}_{\text {obs }}$ should "surround" the domain (this term is specified below) in which the initial data are supported. Therefore, the information contained in the initial condition has a chance to enter the measurement zone. Moreover, our problem formulation will be compatible with the existence of localized defects in the propagation domain. However, to avoid any trapped optic rays, we assume at most one rigid defect which will then be star-shaped in the sense of Definition 2.1.

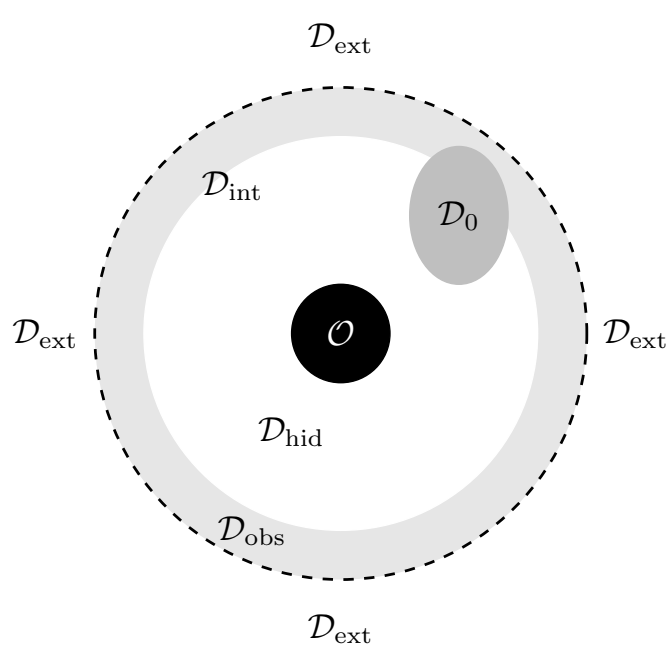

(a)

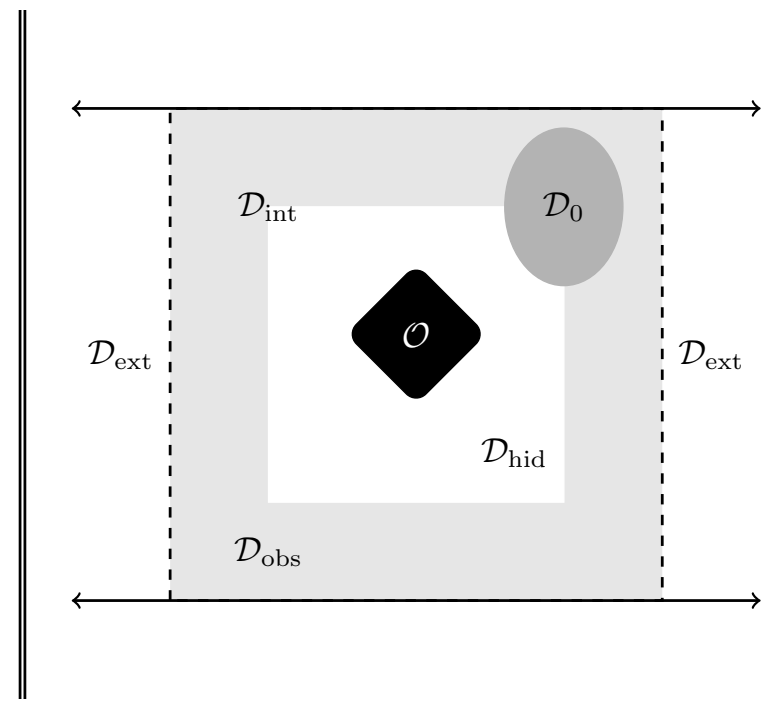

(b)

FiguRe 2. Representation of possible unbounded domains: (a) $\mathcal{D}=\mathbb{R}^{2} \backslash \mathcal{O}$ and (b) $\mathcal{D}=$ $\mathbb{R} \times[0,1] \backslash \mathcal{O}$ where $\mathcal{O}$ is a bounded obstacle represented in black. The observation region $\mathcal{D}_{\text {obs }}$ is represented in grey and the initial data has a compact support included in $\mathcal{D}_{0}$ represented in blue.

Definition 2.1. An open domain $\mathcal{O}$ is called star-shaped if there exists $\mathbf{x}_{0} \in \mathcal{O}$ such that for all $\mathbf{x} \in \mathcal{O}$ the line segment from $\mathbf{x}_{0}$ to $\mathbf{x}$ is included in $\mathcal{O}$.

Our assumption on the domain of propagation are the following:

- the domain of propagation $\mathcal{D}$ is given by $\mathcal{D}=\widetilde{\mathcal{D}} \backslash \overline{\mathcal{O}}$ where $\widetilde{\mathcal{D}}$ is an unbounded open convex subset of $\mathbb{R}^{d}$ and $\mathcal{O}$ is a star-shaped domain with analytic boundaries, bounded and compactly included (see definition below) in $\widetilde{\mathcal{D}}$;

- the initial data $\left(u_{0}, u_{1}\right)$ are compactly supported in a bounded domain $\mathcal{D}_{0} \subset \mathcal{D}$;

- the interior domain (or the domain of interest) $\mathcal{D}_{\text {int }}$ is a convex Lipschitz bounded open domain such that $\mathcal{D}_{0}$ and $\mathcal{O}$ are compactly included in $\mathcal{D}_{\text {int }}$;

- the observation domain $\mathcal{D}_{\text {obs }} \subset \mathcal{D}$ is defined as $\mathcal{D}_{\text {int }} \backslash \overline{\left(\mathcal{D}_{\text {hid }} \cup \mathcal{O}\right)}$, where $\mathcal{D}_{\text {hid }}$ is bounded and compactly included in $\mathcal{D}_{\text {int }}$ and refers to a domain where the measurements are unavailable; 
- finally, we denote by $\mathcal{D}_{\text {ext }}$ the exterior domain defined by $\mathcal{D}_{\text {ext }}=\mathcal{D} \backslash \overline{\mathcal{D}_{\text {int }}}$.

The term compactly included has to be understood in the sense given by Definition 2.2.

Definition 2.2. An open domain $\omega \subset \mathbb{R}^{d}$ is compactly included in a convex open domain $\Omega \subset \mathbb{R}^{d}$ if $\omega \subset \Omega$ and there exists $\varepsilon>0$ such that

$$
\inf _{\mathbf{x} \in \partial \Omega} \inf _{\mathbf{y} \in \partial \omega}|\mathbf{x}-\mathbf{y}|_{2} \geq \varepsilon
$$

where $|\cdot|_{2}$ is the Euclidian norm in $\mathbb{R}^{d}$.

Therefore in fine, we have

$$
\overline{\mathcal{D}}=\overline{\mathcal{D}_{\text {hid }} \cup \mathcal{D}_{\text {obs }} \cup \mathcal{D}_{\text {ext }}}, \quad \mathcal{D}_{\text {hid }} \cap \mathcal{D}_{\text {obs }}=\varnothing, \quad \mathcal{D}_{\text {obs }} \cap \mathcal{D}_{\text {ext }}=\varnothing, \quad \overline{\mathcal{D}_{\text {hid }}} \cap \overline{\mathcal{D}_{\text {ext }}}=\varnothing .
$$

To present our method, we introduce the classical state-space formulation of (1). The state-space is the Hilbert space $\mathcal{Y}=\mathrm{H}_{0}^{1}(\mathcal{D}) \times \mathrm{L}^{2}(\mathcal{D})$ equipped with the scalar product

$$
\forall y_{i}=\left(u_{i}, v_{i}\right) \in \mathcal{Y}, i=1,2, \quad\left(y_{1}, y_{2}\right) \mathcal{Y}=\left(\nabla u_{1}, \nabla u_{2}\right)_{\mathrm{L}^{2}(\mathcal{D})}+\left(v_{1}, v_{2}\right)_{\mathrm{L}^{2}(\mathcal{D})}
$$

The state of the system $y=\left(u, \partial_{t} u\right)$ is the solution to the dynamics

$$
(\mathrm{D})_{0}: \mid \begin{aligned}
& \dot{y}=A y, \quad \text { in }[0, T], \\
& y(0)=y_{0},
\end{aligned}
$$

where in (3), the operator $A$ is an unbounded skew-adjoint operator from $D(A)=D\left(A_{0}\right) \times \mathrm{H}_{0}^{1}(\mathcal{D}) \subset \mathcal{Y}$ into $\mathcal{Y}$ defined by

$$
A=\left[\begin{array}{cc}
0 & \mathbb{1} \\
A_{0} & 0
\end{array}\right],
$$

with $A_{0}: D\left(A_{0}\right) \rightarrow \mathrm{L}^{2}(\mathcal{D})$ defined by

$$
\begin{gathered}
\forall w_{1}, w_{2} \in D\left(A_{0}\right), \quad\left(A_{0} w_{1}, w_{2}\right)_{\mathrm{L}^{2}(\mathcal{D})}=-\left(\nabla w_{1}, \nabla w_{2}\right)_{\mathrm{L}^{2}(\mathcal{D})} \\
D\left(A_{0}\right)=\left\{w \in \mathrm{H}_{0}^{1}(\mathcal{D}) \mid \Delta w \in \mathrm{L}^{2}(\mathcal{D})\right\}
\end{gathered}
$$

We know that $A$ generates a strongly continuous semi-group of contraction - see for instance [7, Prop. 3.1] so that the general dynamics

$$
\text { (D) : } \mid \begin{aligned}
& \dot{y}=A y+f, \quad \text { in }[0, T], \\
& y(0)=y_{0},
\end{aligned}
$$

admits the following solutions:

- if $y_{0} \in \mathcal{Y}$ and $f \in \mathrm{L}^{p}(0, T ; \mathcal{Y})$ problem $(7)$ has a unique mild solution $y$ - in the sense of Duhamel formula - that satisfies

$$
y \in \mathrm{C}^{0}([0, T] ; \mathcal{Y})
$$

- if $y_{0} \in D(A)$ and $f \in \mathrm{W}^{1, p}(0, T ; \mathcal{Y})$ problem $(7)$ has a unique strict solution $y$ that satisfies

$$
y \in \mathrm{C}^{1}([0, T] ; \mathcal{Y}) \cap \mathrm{C}^{0}([0, T] ; D(A))
$$

In our problem, we consider initial conditions of particular structure as we assume that they have a compact support included in $\mathcal{D}_{0}$ hence they belong to a closed subspace of $\mathcal{Y}$, namely

$$
y_{0} \in \mathcal{Y}_{0}=\left\{y \in \mathrm{H}_{0}^{1}(\mathcal{D}) \times \mathrm{L}^{2}(\mathcal{D}) \text { such that }\left.y\right|_{\mathcal{D} \backslash \mathcal{D}_{0}}=0\right\}
$$


To model the available measurements, we introduce a so-called observation operator $C$ from $\mathcal{Y}$ into $\mathcal{Z}=$ $\mathrm{L}^{2}\left(\mathcal{D}_{\text {obs }}\right)$ - linear and bounded in our case - and defined by

$$
C y=\left[\begin{array}{ll}
0 & \mathbb{1}_{\mid \mathcal{D}_{\mathrm{obs}}}
\end{array}\right] y .
$$

We thus assume that there exists a solution $\check{y}$ of $(3)$ - a target trajectory - that has produced the observations, i.e. the measurements,

$$
\forall t \in[0, T], \quad z(t)=C \check{y}(t)+\eta(t),
$$

where $\eta \in \mathrm{L}^{1}(0, T ; \mathcal{Z})$ is a noise assumed to be small. In the state-space formalism, our inverse problem then reads:

Find the unknown $\check{y}_{0} \in \mathcal{Y}$, initial state with support in the given domain $\mathcal{D}_{0}$, of the known dynamics (3), using the noisy observation $z(t)$, available on the known domain $\mathcal{D}_{\text {obs }}$, during time $t \in[0, T]$.

Remark 2.3. Note that keeping in mind the objective of modeling transient elastography problems as envisioned in Figure 1, we have considered Dirichlet boundary conditions on the bounded part of the domain in order to ease the analysis but without really restricting the problem setting with respect to Neumann boundary conditions. Moreover, we could question the fact that velocity measurements are available instead of direct measurements of the field. This assumption implies that the time resolution of the data is sufficient so that we could assume enough time regularity in the data. Note that when this is not the case, [12] gives an alternative observer method in a bounded domain configuration that uses directly the field as a measurement, namely

$$
C y=\left[\mathbb{1}_{\mid \mathcal{D}_{\text {obs }}} \quad 0\right] y .
$$

The convergence is then assessed assuming some $\mathrm{H}^{1}$ space regularity that compensates for the lack of time regularity. Therefore, we could imagine to use directly the field as soon as we are able to extend, for our unbounded case, the data lifting operator introduced in [12], typically in the spirit of [30].

\subsection{An iterative reconstruction strategy}

To solve this inverse problem, we rely on a back-and-forth observer strategy $[2,49]$. Let us introduce the forward observer $\hat{y}$ which is solution to

$$
(\mathrm{F}): \mid \begin{aligned}
& \dot{\hat{y}}=A \hat{y}+\gamma C^{*}(z-C \hat{y}), \quad \text { in }[0, T], \\
& \hat{y}(0)=\hat{y}_{0},
\end{aligned}
$$

where $\gamma$ is a positive constant, $\hat{y}_{0} \in \mathcal{Y}_{0}$ is an initial condition - different from the unknown target initial condition $\check{y}_{0}-$ and $C^{*} \in \mathcal{L}(\mathcal{Z}, \mathcal{Y})$ is the adjoint of $C$ in the sense that

$$
\forall(z, y) \in \mathcal{Z} \times \mathcal{Y}, \quad\left(C^{*} z, y\right)_{\mathcal{Y}}=(z, C y)_{\mathcal{Z}} .
$$

Note that problem (9) is well posed as $A$ is skew-adjoint and $C^{*} C \in \mathcal{L}(\mathcal{Y})$ is positive hence $A_{\gamma}=A-\gamma C^{*} C$ defines a strongly continuous semi-group of contraction. Using Proposition 3.3 in the first chapter of [7] we show that, if $z \in \mathrm{L}^{1}(0, T ; \mathcal{Z})$ then there exists a unique solution $\hat{y} \in \mathrm{C}^{0}([0, T] ; \mathcal{Y})$. We also introduce the backward observer $\hat{y}_{b}$ solution to

$$
\text { (B) : } \mid \begin{aligned}
& \dot{\hat{y}}_{b}=A \hat{y}_{b}-\gamma C^{*}\left(z-C \hat{y}_{b}\right), \quad \text { in }[0, T], \\
& \hat{y}_{b}(T)=\hat{y}(T) .
\end{aligned}
$$

Note that this problem is initialized at the final time $T$ and is solved in reverse time (hence the minus sign multiplying $\left.C^{*} C\right)$. It is well posed since, $\left(A_{\gamma}\right)^{*}=-A-\gamma C^{*} C$ is maximal dissipative hence - by the Lumer and Phillips theorem $-\left(A_{\gamma}\right)^{*}$ is the generator of a strongly continuous semi-group of contraction [7, Chapter 1,Theorem 2.8]. Therefore, there exists a unique solution $\hat{y}_{b} \in \mathrm{C}^{0}([0, T] ; \mathcal{Y})$. Our objective is to justify that 
the observers $\hat{y}$ and $\hat{y}_{b}$ are converging - at least with perfect measurements - to the target state $\check{y}$ as time increases for $\hat{y}$ and as time decreases for $\hat{y}_{b}$ thanks to the correcting terms $\gamma C^{*}(z-C \hat{y})$ and $-\gamma C^{*}\left(z-C \hat{y}_{b}\right)$. Thus, we expect $\hat{y}_{b}(0)$ to be a better approximation of the initial state $\check{y}_{0}$ than the initial guess $\hat{y}(0)$. We can then envision to iterate this process by re-initializing the forward observer with this new initial guess leading to the following back-and-forth nudging algorithm: for $n \geq 0$

$$
(\mathrm{B} \& \mathrm{~F}): \mid \begin{array}{ll}
\dot{\hat{y}}^{(n)}=A \hat{y}^{(n)}+\gamma C^{*}\left(z-C \hat{y}^{(n)}\right), & \text { in }[0, T] \\
\hat{y}^{(n)}(0)=\hat{y}_{b}^{(n-1)}(0), & \\
\dot{\hat{y}}_{b}^{(n)}=A \hat{y}_{b}^{(n)}-\gamma C^{*}\left(z-C \hat{y}_{b}^{(n)}\right), & \text { in }[0, T] \\
\hat{y}_{b}^{(n)}(T)=\hat{y}^{(n)}(T),
\end{array}
$$

this algorithm being initialized by

$$
\hat{y}_{b}^{(-1)}(0)=\hat{y}_{0} \text {. }
$$

Yet, as we will see in Section 3, we cannot prove the convergence of the above algorithm due to the fact that the sequence of initial state $\hat{y}^{(n)}(0)$ is not compactly supported in $\mathcal{D}_{0}$. To get a convergent algorithm, we must add a projection step that ensures that the initial state $\hat{y}^{(n)}(0)$ is compactly supported in $\mathcal{D}_{0}$. Then, the algorithm becomes

$$
(\mathrm{B \& F}+\text { Proj }): \mid \begin{array}{ll}
\dot{\hat{y}}^{(n)}=A \hat{y}^{(n)}+\gamma C^{*}\left(z-C \hat{y}^{(n)}\right), \quad \text { in }[0, T], \\
\hat{y}^{(n)}(0)=\Pi \hat{y}_{b}^{(n-1)}(0), \\
\hat{\hat{y}}_{b}^{(n)}=A \hat{y}_{b}^{(n)}-\gamma C^{*}\left(z-C \hat{y}_{b}^{(n)}\right), \quad \text { in }[0, T], \\
\hat{y}_{b}^{(n)}(T)=\hat{y}^{(n)}(T),
\end{array}
$$

where $\Pi$ is an orthonormal projection operator from $\mathcal{Y}$ into $\mathcal{Y}_{0}$. An explicit formulation of the projection $\Pi$ is as follows: for all $y=(u, v)$ we set

$$
\Pi y=\left(u_{0},\left.v\right|_{\mathcal{D}_{0}}\right) \text { with } u_{0} \in \mathrm{H}_{0}^{1}\left(\mathcal{D}_{0}\right) \text { and } \Delta u_{0}=\Delta u \text { in } \mathcal{D}_{0}
$$

and where $u_{0}$ is extended by 0 outside $\mathcal{D}_{0}$. We want here to emphasize that this projection step is a mandatory step that was not required for back-and-forth observer strategies for wave equations in bounded domains [49]. Considering our algorithm (B\&F - Proj) given by (12), let us consider the following criterion

Criterion 2.4 (Observability condition). There exists $T>0$ and $\kappa>0$ such that, for all initial state $y_{0} \in \mathcal{Y}_{0}$

$$
\int_{0}^{T}\left\|C e^{A s} y_{0}\right\|_{\mathcal{Z}}^{2} \mathrm{~d} t \geq \kappa\left\|y_{0}\right\|_{\mathcal{Y}}^{2}
$$

This criterion conditions the next theorem.

Theorem 2.5. If the Observability Condition 2.4 holds then, in the algorithm (12), we have

- in the absence of noise, $\hat{y}^{(n)}(0)$ converges exponentially fast to $\check{y}_{0}$, namely there exists $\alpha<1$ such that

$$
\left\|\hat{y}^{(n)}(0)-\check{y}_{0}\right\| \mathcal{Y} \leq \alpha^{n}\left\|\check{y}_{0}-\hat{y}_{0}\right\|_{\mathcal{Y}}
$$

- if we consider noisy data $z(t)=C \check{y}(t)+\eta(t)$ where $\eta \in \mathrm{L}^{1}(0, T ; \mathcal{Z})$, then we have, for the same $\alpha<1$, the error bound

$$
\left\|\hat{y}^{(n)}(0)-\check{y}_{0}\right\| \mathcal{Y} \leq \alpha^{n}\left\|\check{y}_{0}-\hat{y}_{0}\right\| \mathcal{Y}+\frac{2 \gamma}{1-\alpha} \int_{0}^{T}\|\eta(s)\|_{\mathcal{Z}} \mathrm{d} s
$$

This theorem deserves some comments: 
- We will show that $\alpha$ is in fact the norm of the reconstruction operator. We see that the closer $\alpha$ is to 0 (at least when $\eta=0$ ), the better our estimate is. So, it seems natural to seek the best $\gamma$ parameter so that $\alpha$ is the closest to 0 .

- If the observability inequality is not satisfied, then it can be shown that $\alpha=1$ and our error estimate blows up in the presence of noise (if $\gamma \neq 0$ ). In [30], a weaker convergence for a similar problem - although in the absence of noise - has been obtained. The weaker stability is based only on the study of the injectivity of the operator

$$
\Psi_{T}:[0, T] \ni t \mapsto C e^{A t} y_{0},
$$

whereas (14) is a coercivity property for $\Psi_{T}$. Note that, here, we are able to prove the observability condition which then allows the exponential stability, and then an analysis of the noise impact. This would have been much more intricate with a weaker stability obtained by an injectivity condition only.

- In [50] and [30] strategies are presented with the main difference that data are assumed to be given only on the boundary of the domain of interest. It would correspond for instance to data being given on $\partial \mathcal{H}_{\text {obs }}$ where $\mathcal{H}_{\text {obs }}$ is the convex hull of $\mathcal{D}_{\text {obs }}$. Although it seems less restrictive that what we have presented, where data are given in all $\mathcal{D}_{\text {obs }}$, the exponential convergence of the algorithm is much more intricate or even not possible to prove. Moreover, the stability analysis of the algorithm presented in [50] suggests that the measurements $u(t)$ of the wave equation solution should belong to $\mathrm{H}^{1}\left((0, T) \times \partial \mathcal{H}_{\text {obs }}\right)$ (compared to $\mathrm{W}^{1,1}\left(0, T ; \mathrm{L}^{2}\left(\mathcal{D}_{\text {obs }}\right)\right)$ in our case).

The proof of Theorem (2.5) will be given all along Section 3. The convergence of the algorithm is studied in Section 3.1 by the introduction of a contracting operator that is applied at each iteration of the algorithm. The robustness of the algorithm with respect to noise will be given in Section 3.2. Finally, as our results rely on the observability inequality (14), in Section 3.3, it is proved to hold in the setting described Section 2.1.

\section{Convergence Analysis}

\subsection{The reconstruction-error operator}

To analyze the convergence of the algorithm (12), we first consider a noise-free configuration with $\eta \equiv 0$. Let us introduce the forward error state $\tilde{y}=\hat{y}-\check{y}$ solution to, in the absence of noise,

$$
(\text { F-err }): \mid \begin{aligned}
& \dot{\tilde{y}}^{(n)}=A \tilde{y}^{(n)}-\gamma C^{*} C \tilde{y}^{(n)}, \quad \text { in }[0, T], \\
& \tilde{y}^{(n)}(0)=\Pi \tilde{y}_{b}^{(n-1)}(0),
\end{aligned}
$$

and the backward error state $\tilde{y}_{b}=\hat{y}_{b}-\check{y}$ solution to, in the absence of noise,

$$
\text { (B-err) : } \begin{aligned}
& \dot{\tilde{y}}_{b}^{(n)}=A \tilde{y}_{b}^{(n)}+\gamma C^{*} C \tilde{y}_{b}^{(n)}, \quad \text { in }[0, T], \\
& \tilde{y}_{b}^{(n)}(T)=\tilde{y}^{(n)}(T) .
\end{aligned}
$$

Since $\gamma>0, A_{\gamma}=A-\gamma C^{*} C$ generates a dissipative semi-group of operator [7] and the solution $\tilde{y}$ of (17) is given by

$$
\tilde{y}^{(n)}(t)=e^{A_{\gamma} t} \tilde{y}^{(n)}(0) .
$$

Similarly, we get for the backward error state that $\tilde{y}_{b}$ is given by

$$
\tilde{y}_{b}^{(n)}(t)=e^{A_{\gamma}^{*}(T-t)} \tilde{y}_{b}^{(n)}(T) .
$$

Let us now define $\Lambda_{\gamma}$, the linear reconstruction-error operator, that maps the initial state error $\tilde{y}_{0}$ to $\tilde{y}_{b}(0)$. From (19) and (20), we have the explicit characterization

$$
\Lambda_{\gamma}: \mathcal{Y} \ni y \mapsto \Lambda_{\gamma} y=e^{A_{\gamma}^{*} T} e^{A_{\gamma} T} y \in \mathcal{Y} .
$$


We then have for all $n \in \mathbb{N}^{*}$ the following reconstruction formula

$$
\begin{aligned}
\tilde{y}^{(n)}(0) & =\hat{y}^{(n)}(0)-y_{0}=\Pi \hat{y}_{b}^{(n-1)}(0)-\Pi y_{0} \\
& =\Pi \tilde{y}_{b}^{(n-1)}(0)=\Pi \Lambda_{\gamma} \tilde{y}^{(n-1)}(0) .
\end{aligned}
$$

Recalling that $\tilde{y}^{(n-1)}(0) \in \mathcal{Y}_{0}$ hence that $\Pi \tilde{y}^{(n-1)}(0)=\tilde{y}^{(n-1)}(0)$, we finally obtain

$$
\forall n \geq 1, \quad \tilde{y}^{(n)}(0)=\Pi \Lambda_{\gamma} \Pi \tilde{y}^{(n-1)}(0) .
$$

Therefore, the convergence of (12), is related to a contracting property of the operator $\Pi \Lambda_{\gamma} \Pi$. Let us then better specify $\Lambda_{\gamma}$.

Proposition 3.1. The operator $\Lambda_{\gamma}$ is a bounded self-adjoint positive operator from $\mathcal{Y}$ to $\mathcal{Y}$ defined by

$$
\Lambda_{\gamma}=\mathbb{1}-2 \gamma \int_{0}^{T} e^{A_{\gamma}^{*} s} C^{*} C e^{A_{\gamma} s} \mathrm{~d} s,
$$

where $\mathbb{1}$ denotes the identity operator. Moreover, $\left\|\Lambda_{\gamma}\right\|_{\mathcal{L}(\mathcal{Y}, \mathcal{Y})} \leq 1$.

Proof. Using (21), we easily deduce that the operator $\Lambda_{\gamma}$ is symmetric and positive since, from (21), we have

$$
\left(\Lambda_{\gamma} y, y\right)_{\mathcal{Y}}=\left\|e^{A_{\gamma} T} y\right\|_{\mathcal{Y}}^{2} \geq 0
$$

To show (24), let us first assume that $\tilde{y}_{0}$ is sufficiently smooth, namely $\tilde{y}_{0} \in D(A)$, so that $\tilde{y} \in \mathrm{C}^{0}([0, T], D(A)) \cap$ $\mathrm{C}^{1}([0, T], \mathcal{Y})$. Then, using (21) and Stokes' formula we get that

$$
\begin{aligned}
\Lambda_{\gamma} \tilde{y}(0) & =\tilde{y}(0)+\int_{0}^{T} \partial_{s}\left(e^{A_{\gamma}^{*} s} e^{A_{\gamma} s}\right) \tilde{y}(0) \mathrm{d} s \\
& =\tilde{y}(0)+\int_{0}^{T} e^{A_{\gamma}^{*} s}\left(A_{\gamma}^{*}+A_{\gamma}\right) e^{A_{\gamma} s} \tilde{y}(0) \mathrm{d} s \\
& =\tilde{y}(0)-2 \gamma \int_{0}^{T} e^{A_{\gamma}^{*} s} C^{*} C e^{A_{\gamma} s} \tilde{y}(0) \mathrm{d} s,
\end{aligned}
$$

recalling that $A_{\gamma}=A-\gamma C^{*} C$. Finally, to prove that the norm of $\Lambda_{\gamma}$ is less or equal to one, we proceed in two steps. First, setting $\tilde{y}_{0}=\tilde{y}(0)$, we note that

$$
0 \leq\left(\Lambda_{\gamma} \tilde{y}_{0}, \tilde{y}_{0}\right)_{\mathcal{Y}}=\left\|\tilde{y}_{0}\right\|_{\mathcal{Y}}^{2}-2 \underbrace{2 \gamma \int_{0}^{T}\left\|C e^{A_{\gamma} s} \tilde{y}_{0}\right\|_{\mathcal{Z}}^{2} \mathrm{~d} s}_{\geq 0} \leq\left\|\tilde{y}_{0}\right\|_{\mathcal{Y}}^{2} .
$$

Second, we introduce the (self-adjoint) square root $\Lambda_{\gamma}^{\frac{1}{2}}$ of the positive self-adjoint operator $\Lambda_{\gamma}$. We get

$$
\left\|\Lambda_{\gamma} \tilde{y}_{0}\right\|_{\mathcal{Y}}^{2}=\left(\Lambda_{\gamma} \tilde{y}_{0}, \Lambda_{\gamma} \tilde{y}_{0}\right)_{\mathcal{Y}}=\left(\Lambda_{\gamma} \Lambda_{\gamma}^{\frac{1}{2}} \tilde{y}_{0}, \Lambda_{\gamma}^{\frac{1}{2}} \tilde{y}_{0}\right)_{\mathcal{Y}}
$$

Using (25), it comes

$$
\left\|\Lambda_{\gamma} \tilde{y}_{0}\right\|_{\mathcal{Y}}^{2} \leq\left\|\Lambda_{\gamma}^{\frac{1}{2}} \tilde{y}_{0}\right\|_{\mathcal{Y}}^{2}=\left(\Lambda_{\gamma} \tilde{y}_{0}, \tilde{y}_{0}\right)_{\mathcal{Y}} \leq\left\|\tilde{y}_{0}\right\|_{\mathcal{Y}}^{2}
$$

which proves the result. We extend the result for all $\tilde{y}_{0} \in \mathcal{Y}$ by density of $D(A)$ into $\mathcal{Y}$.

Since $\Pi$ is a projector from $\mathcal{Y}$ to $\mathcal{Y}_{0}$, we deduce from Proposition 3.1 and (23) that the error in (12) cannot grow as it is controlled by the initial error. In fact, we go further and prove in the next proposition that the error strictly decreases. 
Proposition 3.2. The operator $\Pi \Lambda_{\gamma} \Pi$ is bounded, self-adjoint and positive. Moreover, if the Observability Condition 2.4 of is satisfied, then $\left\|\Pi \Lambda_{\gamma} \Pi\right\|_{\mathcal{L}(\mathcal{Y}, \mathcal{Y})}<1$.

Proof. First, let us recall that $\Pi$ and $\Lambda_{\gamma}$ are self-adjoint operators, so it is clear that $\Pi \Lambda_{\gamma} \Pi$ is symmetric. Moreover, for all $y \in \mathcal{Y}$, we have

$$
\left(\Pi \Lambda_{\gamma} \Pi y, y\right)_{\mathcal{Y}}=\left(\Lambda_{\gamma} \Pi y, \Pi y\right)_{\mathcal{Y}} \geq 0 .
$$

Then, we deduce from (24) that

$$
0 \leq\left(\Lambda_{\gamma} \Pi y, \Pi y\right)_{\mathcal{Y}}=\|\Pi y\|_{\mathcal{Y}}^{2}-2 \gamma \int_{0}^{T}\left\|C e^{A_{\gamma} s} \Pi y\right\|_{\mathcal{Z}}^{2} \mathrm{~d} s .
$$

Let us now assume that there exists a constant $\tilde{\kappa}>0$ independent of $y$ such that

$$
\int_{0}^{T}\left\|C e^{A_{\gamma} s} \Pi y\right\|_{\mathcal{Z}}^{2} \mathrm{~d} s \geq \tilde{\kappa}\|\Pi y\|_{\mathcal{Y}}^{2}
$$

In this case, we have that

$$
\begin{aligned}
0 \leq\left(\Lambda_{\gamma} \Pi y, \Pi y\right)_{\mathcal{Y}} & \leq(1-2 \gamma \tilde{\kappa})\|\Pi y\|_{\mathcal{Y}}^{2} \\
& \leq \underbrace{(1-2 \gamma \tilde{\kappa})}_{<1}\|y\|_{\mathcal{Y}}^{2} .
\end{aligned}
$$

We would then conclude as in (26) by introducing the square root $\left(\Pi \Lambda_{\gamma} \Pi\right)^{\frac{1}{2}}$ and using (28) so that we have

$$
\begin{aligned}
\left\|\Pi \Lambda_{\gamma} \Pi \tilde{y}_{0}\right\|_{\mathcal{Y}}^{2} & =\left(\left(\Pi \Lambda_{\gamma} \Pi\right)\left(\Pi \Lambda_{\gamma} \Pi\right)^{\frac{1}{2}} y,\left(\Pi \Lambda_{\gamma} \Pi\right)^{\frac{1}{2}} y\right)_{\mathcal{Y}} \\
& \leq(1-2 \gamma \tilde{\kappa})\left\|\left(\Pi \Lambda_{\gamma} \Pi\right)^{\frac{1}{2}} y\right\|_{\mathcal{Y}}^{2} \\
& \leq(1-2 \gamma \tilde{\kappa})\left(\left(\Pi \Lambda_{\gamma} \Pi\right) y, y\right)_{\mathcal{Y}} \\
& \leq(1-2 \gamma \tilde{\kappa})^{2}\|y\|_{\mathcal{Y}}^{2} .
\end{aligned}
$$

Therefore to conclude our proof, it only remains to prove that the observability inequality (14) implies the observability inequality (27), with $\tilde{\kappa} \leq \kappa$. This is a classical result when $C$ is a bounded operator, see for instance [53, Theorem 1.3], namely if $(A, C)$ is observable, then $\left(A_{\gamma}, C\right)$ is also observable.

Proposition 3.2 shows the convergence of the algorithm (23) and therefore proves the first part of Theorem 2.5. Indeed, by (23), we get

$$
\begin{aligned}
\left\|\tilde{y}^{(n)}(0)\right\|_{\mathcal{Y}} & =\left\|\Pi \Lambda_{\gamma} \Pi \tilde{y}^{(n-1)}(0)\right\|_{\mathcal{Y}} \\
& \leq \alpha\left\|\tilde{y}^{(n-1)}(0)\right\|_{\mathcal{Y}} \leq \alpha^{n}\left\|\tilde{y}^{(0)}(0)\right\| .
\end{aligned}
$$

where $\alpha=\left\|\Pi \Lambda_{\gamma} \Pi\right\|_{\mathcal{L}(\mathcal{Y}, \mathcal{Y})}<1$. In the absence of noise, our strategy is then converging exponentially fast.

\subsection{Noisy data analysis}

In this section, we now discuss the robustness of the algorithm (12) with respect to noisy data. We thus denote by $\eta(t) \in \mathcal{Z}$ the difference between the measurements at hand and the measurements that would have been produced by the target trajectory $\check{y}$. In other words, the data considered $z(t)$ is given by

$$
\forall t \in[0, T], \quad z(t)=C \check{y}(t)+\eta(t),
$$


and we assume that $\eta \in \mathrm{L}^{1}(0, T ; \mathcal{Z})$. We want to assess if our reconstruction algorithm (12) still converges, with a controlled error with respect to some norm on $\eta$.

Considering the observation given by (29), the error $\tilde{y}=\hat{y}-\check{y}$ now satisfies

$$
\left(\mathrm{F}_{\eta^{-}} \text {err }\right): \mid \begin{aligned}
& \dot{\tilde{y}}=A \tilde{y}-\gamma C^{*} C \tilde{y}+\gamma C^{*} \eta, \quad \text { in } \quad[0, T], \\
& \tilde{y}(0)=\tilde{y}_{0} .
\end{aligned}
$$

The term $\gamma C^{*} \eta$ acts as a source term, and using Duhamel's formula we have

$$
\tilde{y}(t)=e^{A_{\gamma} t} \tilde{y}_{0}+\gamma \int_{0}^{t} e^{A_{\gamma}(t-s)} C^{*} \eta(s) \mathrm{d} s .
$$

Similarly, the error $\tilde{y}_{b}$ satisfies

$$
\left(\mathrm{B}_{\eta^{-}} \mathrm{err}\right): \mid \begin{aligned}
& \dot{\tilde{y}}_{b}=A \tilde{y}_{b}+\gamma C^{*} C \tilde{y}_{b}-\gamma C^{*} \eta \quad \text { in } \quad[0, T], \\
& \tilde{y}_{b}(T)=\tilde{y}(T)
\end{aligned}
$$

and we get, thanks to Duhamel's formula (reverse in time):

$$
\tilde{y}_{b}(t)=e^{A_{\gamma}^{*}(T-t)} \tilde{y}_{b}(T)+\gamma \int_{t}^{T} e^{A_{\gamma}^{*}(s-t)} C^{*} \eta(s) \mathrm{d} s .
$$

Then combining (31) and (33), we get

$$
\tilde{y}_{b}(0)=\Lambda_{\gamma} \tilde{y}_{0}+\gamma e^{A_{\gamma}^{*} T} \int_{0}^{T} e^{A_{\gamma}(T-s)} C^{*} \eta(s) \mathrm{d} s+\gamma \int_{0}^{T} e^{A_{\gamma}^{*} s} C^{*} \eta(s) \mathrm{d} s .
$$

Recalling that $\Lambda_{\gamma}=e^{A_{\gamma}^{*} T} e^{A_{\gamma} T}$ (see (21)), we then deduce that the error in Algorithm (12) is given by

$$
\tilde{y}^{(n+1)}(0)=\Pi\left(\Lambda_{\gamma} \Pi \tilde{y}^{(n)}(0)+\gamma\left(\int_{0}^{T} e^{A_{\gamma}^{*} s} C^{*} \eta(s) \mathrm{d} s+e^{A_{\gamma}^{*} T} \int_{0}^{T} e^{A_{\gamma}(T-s)} C^{*} \eta(s) \mathrm{d} s\right)\right) .
$$

Then a triangular inequality gives

$$
\left\|\tilde{y}^{(n+1)}(0)\right\|_{\mathcal{Y}} \leq\left\|\Pi \Lambda_{\gamma} \Pi \tilde{y}^{(n)}(0)\right\|_{\mathcal{Y}}+\gamma\left(\int_{0}^{T}\left\|e^{A_{\gamma}^{*} s} C^{*} \eta(s)\right\|_{\mathcal{Y}} \mathrm{d} s+\left\|e^{A_{\gamma}^{*} T} \int_{0}^{T} e^{A_{\gamma}^{*}(T-s)} C^{*} \eta(s) \mathrm{d} s\right\|_{\mathcal{Y}}\right) .
$$

The operators $A_{\gamma}$ and $A_{\gamma}^{*}$ generate semi-groups of contraction, hence we have

$$
\left\|e^{A_{\gamma}^{*} s} C^{*} \eta(s)\right\|_{\mathcal{Y}} \leq\left\|C^{*} \eta(s)\right\|_{\mathcal{Y}}=\|\eta(s)\|_{\mathcal{Z}},
$$

and

$$
\left\|e^{A_{\gamma}^{*} T} \int_{0}^{T} e^{A_{\gamma}(T-s)} C^{*} \eta(s) \mathrm{d} s\right\|_{\mathcal{Y}} \leq \int_{0}^{T}\|\eta(s)\|_{\mathcal{Z}} \mathrm{d} s .
$$

We know that if the observability inequality is satisfied, Proposition 3.2 gives that $\left\|\Pi \Lambda_{\gamma} \Pi\right\|=\alpha<1$. Thus, we deduce that

$$
\left\|\tilde{y}^{(n+1)}(0)\right\|_{\mathcal{Y}} \leq \alpha\left\|\tilde{y}^{(n)}(0)\right\|_{\mathcal{Y}}+2 \gamma \int_{0}^{T}\|\eta(s)\|_{\mathcal{Z}} \mathrm{d} s,
$$

from which we get by induction

$$
\left\|\tilde{y}^{(n)}(0)\right\| \mathcal{Y} \leq \alpha^{n}\left\|\tilde{y}^{(0)}(0)\right\|_{\mathcal{Y}}+2 \gamma\left(\sum_{j=0}^{n} \alpha^{j}\right) \int_{0}^{T}\|\eta(s)\|_{\mathcal{Z}} \mathrm{d} s .
$$


Finally, noticing that

$$
\sum_{j=0}^{n} \alpha^{j}=\frac{1-\alpha^{n+1}}{1-\alpha} \leq \frac{1}{1-\alpha}
$$

we get

$$
\left\|\tilde{y}^{(n)}(0)\right\|_{\mathcal{Y}} \leq \alpha^{n}\left\|\tilde{y}^{(0)}(0)\right\|_{\mathcal{Y}}+\frac{2 \gamma}{1-\alpha} \int_{0}^{T}\|\eta(s)\|_{\mathcal{Z}} \mathrm{d} s .
$$

The above inequality proves that the algorithm (12) converges to a ball of center the target initial condition and of radius controlled by the noise norm in $\mathrm{L}^{1}(0, T ; \mathcal{Z})$. This results completes the proof of Theorem 2.5 .

Remark 3.3. Note that more general configurations can be considered with $\eta$ belonging to a less regular space. This will lead to the necessary introduction of regularization strategies on the data or on the reconstruction - as in every inverse problem [19] - to be combined with the result presented in this section. Moreover, we have limited our approach to a deterministic treatment of the noise as a first step but stochastic perturbations could also be envisioned in the observer framework [6].

Remark 3.4. We expect that Theorem 2.5, could be adapted to other types of "hyperbolic" equations - namely transport equations, Schrodinger equations, elastodynamics systems - as the proof depends essentially of the skew-adjointness of the generator $A$. However, it will remain necessary to have the Observability Condition 2.4 for each problem, a result which is always very dependent of the underlying equation. By contrast, the next section is therefore very dependent of the wave equation of interest in the present paper.

\subsection{The observability inequality}

Our objective in this section is to prove the observability inequality (14), hence to justify the condition of application of Theorem 2.5. It is worth noticing that - since the observation region $\mathcal{D}_{\text {obs }}$ is assumed to be bounded - it is clear that inequality (14) cannot be satisfied for any initial state. However here, our objective is to show that we can prove the observability condition for initial condition of support in $\mathcal{D}_{\text {hid }}$ surrounded by the observation domain.

In this respect, to prove (14) we rely on the two following geometrical consequences of the assumptions given Section 2.1:

- the domain $\mathcal{D}_{\text {gcc }}=\overline{\mathcal{D}_{\text {obs }} \cup \mathcal{D}_{\text {ext }}}$ surrounds the domain $\mathcal{D}_{\text {hid }}$ with

$$
\overline{\mathcal{D}}=\overline{\mathcal{D}_{\text {hid }}} \cup \overline{\mathcal{D}_{\text {gcc }}}, \quad \partial \mathcal{D}_{\text {hid }} \cap \partial \mathcal{D}=\partial \mathcal{O} .
$$

Moreover, the obstacle $\mathcal{O}$ is star-shaped with analytic boundaries. Thus, any ray touching $\mathcal{O}$ will escape $\mathcal{D}_{\text {hid }}$ to enter $\mathcal{D}_{\text {gcc }}$. Therefore, it is the case for any rays from $\mathcal{D}_{\text {hid }}$. Then, $\mathcal{D}_{\text {gcc }}$ satisfies the exterior geometrical control condition (GCCE) [10] in the strong sense precised by [11]. This means that, for $T$ sufficiently large, every geometric optics ray in $\mathcal{D}$ intersects the domain $\mathcal{D}_{\text {gcc }}-$ in the sense that if a ray starting at $\xi$ is parametrized by $\left\{x_{\xi}(s), s \in[0, T]\right\}$, we have

$$
\forall \xi \in \mathcal{D}, \exists s \in(0, T), \exists \delta>0, B\left(x_{\xi}(s), \delta\right) \subset \mathcal{D}_{\text {gcc }}
$$

- there exists an open subdomain $\mathcal{D}_{c} \subset \mathcal{D}_{\text {obs }}$ such that we can define positive $\mathrm{C}^{2}(\mathcal{D})$ functions $\chi_{c}$ and $\xi_{c}$ that satisfy (see Figure 3 for an illustrative example)

$$
\chi_{c}=\left\{\begin{array}{lll}
1 & \text { in } & \mathcal{D}_{\text {ext }}, \\
0 & \text { in } & \mathcal{D}_{0} \cup \partial \mathcal{O},
\end{array}, \quad \nabla \chi_{c}=0 \text { in } \mathcal{D} \backslash \mathcal{D}_{c},\right.
$$

and

$$
\xi_{c}=\left\{\begin{array}{lll}
1 & \text { in } & \mathcal{D}_{c} \\
0 & \text { in } \quad & \left(\mathcal{D} \backslash \mathcal{D}_{\text {obs }}\right) \cup \mathcal{D}_{0} \cup \partial \mathcal{O} \\
& & 12
\end{array}\right.
$$


Moreover in the following, we will consider a norm of the solutions defined in some sub-domains only. Therefore, we introduce the notation: for any $y=(u, v) \in \mathcal{Y}$,

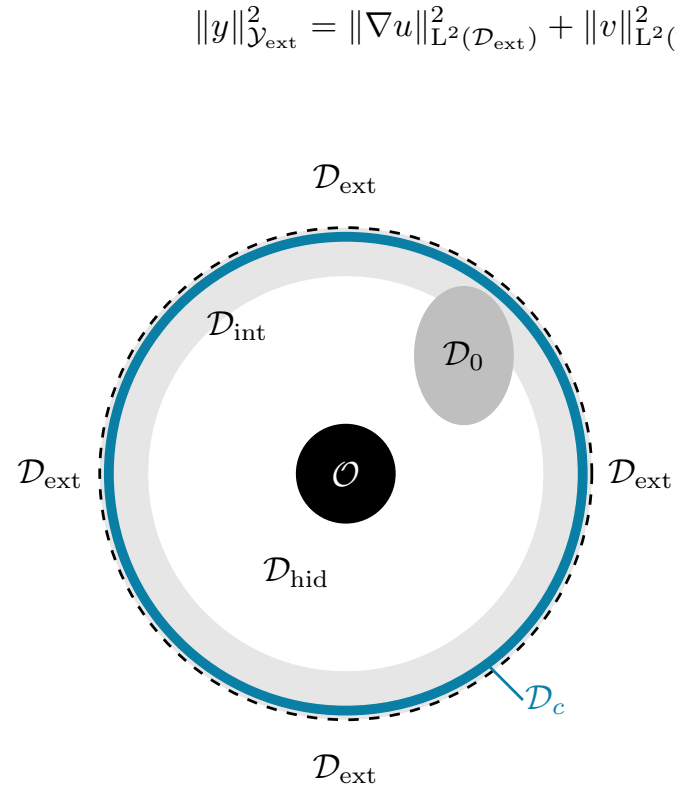

(a)

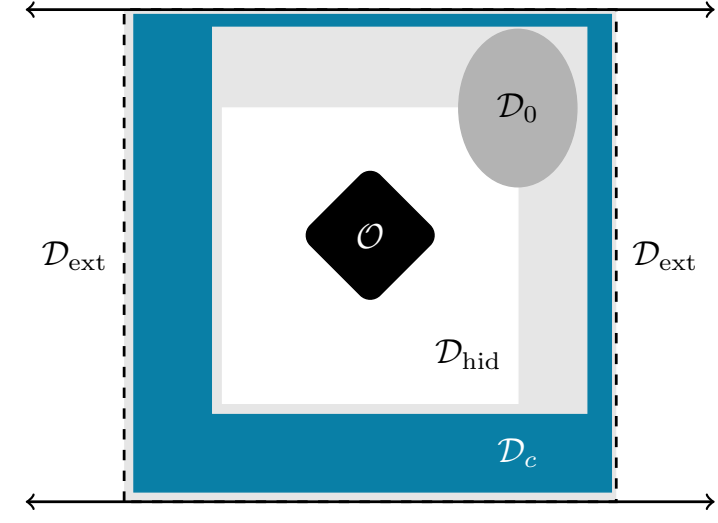

(b)

FIgURE 3. Representation of the domain $\mathcal{D}_{c}$ for the geometry presented in Figure 2

We will then prove the following proposition.

Proposition 3.5. There exists $T>0$ and $\kappa>0$ such that for any initial state $y_{0} \in \mathcal{Y}_{0}$ we have

$$
\int_{0}^{T}\left\|C e^{A s} y_{0}\right\|_{\mathcal{Z}}^{2} \mathrm{~d} s \geq \kappa \int_{0}^{T}\left\|e^{A s} y_{0}\right\|_{\mathcal{Y}_{\text {ext }}}^{2} \mathrm{~d} s
$$

This proposition shows that the norm of the solution in the exterior domain $\mathcal{D}_{\text {ext }}$ is controlled by the measurement in $\mathcal{D}_{\text {obs }}$ if the initial state is supported in $\mathcal{D}_{0}$. Before proving this result, let us explain how

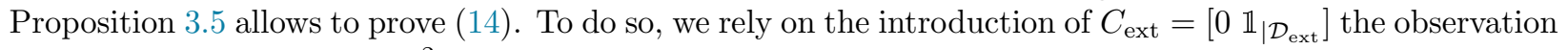
operator from $\mathcal{Y} \rightarrow \mathcal{Z}_{\text {ext }}=\mathrm{L}^{2}\left(\mathcal{D}_{\text {ext }}\right)$ associated with the exterior domain $\mathcal{D}_{\text {ext }}$. With the second geometrical assumption, we get the following proposition.

Proposition 3.6. Under the Strong Geometrical Control Condition (37) for the domain $\mathcal{D}_{\mathrm{gcc}}$, the following observability inequality holds: There exists $T>0$ and $\tilde{\kappa}>0$ sufficiently large such that, for any initial state $y_{0} \in \mathcal{Y}$, we have

$$
\int_{0}^{T}\left\|C_{\mathrm{ext}} e^{A s} y_{0}\right\|_{\mathrm{L}^{2}\left(\mathcal{D}_{\text {ext }}\right)}^{2}+\left\|C e^{A s} y_{0}\right\|_{\mathcal{Z}}^{2} \mathrm{~d} s \geq \tilde{\kappa}\left\|y_{0}\right\|_{\mathcal{Y}}^{2} .
$$

The proof of this result can be found for instance in [10] with the complement on the strong GCC in [11]. Let us remark that it corresponds to the situation where the observation region $\mathcal{D}_{\text {obs }} \cup \mathcal{D}_{\text {ext }}$ is unbounded. Combining these last two propositions, it is easy to deduce that the observability inequality (14) is satisfied. Indeed, on the one hand, we get by Proposition 3.5

$$
\begin{aligned}
\int_{0}^{T}\left\|C e^{A s} y_{0}\right\|_{\mathcal{Z}}^{2} \mathrm{~d} s & \geq \kappa \int_{0}^{T}\left\|e^{A s} y_{0}\right\|_{\mathcal{Y}_{\text {ext }}}^{2} \mathrm{~d} s \\
& \geq \kappa \int_{0}^{T}\left\|C_{\text {ext }} e^{A s} y_{0}\right\|_{\mathrm{L}^{2}\left(\mathcal{D}_{\text {ext }}\right)}^{2} \mathrm{~d} s
\end{aligned}
$$


since $C_{\text {ext }}$ has a norm equal to one. On the other hand, we get by Proposition 3.6

$$
\begin{aligned}
\left(\frac{1}{\kappa}+1\right) \int_{0}^{T}\left\|C e^{A s} y_{0}\right\|_{\mathcal{Z}}^{2} \mathrm{~d} s & \geq \int_{0}^{T}\left\|C_{\text {ext }} e^{A s} y_{0}\right\|_{L^{2}\left(\mathcal{D}_{\text {ext }}\right)}^{2}+\left\|C e^{A s} y_{0}\right\|_{\mathcal{Z}}^{2} \mathrm{~d} s \\
& \geq \tilde{\kappa}\left\|y_{0}\right\|_{\mathcal{Y}}^{2} .
\end{aligned}
$$

Coming back to the proof of Proposition 3.5, we need two preliminary lemmas. The first one is related to the propagation of the energy to the exterior domain $\mathcal{D}_{\text {ext }}$ through the layer $\mathcal{D}_{c}$, whereas the second one is related to an energy equidistribution property in the observation domain during time. Then finally, Proposition 3.5 will be a direct consequence of equations (41) and (46) given in the two aforementioned lemmas.

Lemma 3.7. There exists $T>0$ and $\kappa_{a}>0$ such that for any initial state $y_{0} \in \mathcal{Y}_{0}$

$$
\int_{0}^{T}\left\|e^{A s} y_{0}\right\|_{\mathcal{Y}_{c}}^{2} \mathrm{~d} s \geq \kappa_{a} \int_{0}^{T}\left\|e^{A s} y_{0}\right\|_{\mathcal{Y}_{\text {ext }}}^{2} \mathrm{~d} s
$$

Proof. Recalling that $y(s)=e^{A s} y_{0}$, is solution to $\dot{y}=A y$ we get by using $y_{c}=\left(\chi_{c} u, \chi_{c} v\right) \in \mathcal{Y}$ as a multiplier,

$$
\left(\dot{y}, y_{c}\right)_{\mathcal{Y}}=\left(A y, y_{c}\right)_{\mathcal{Y}}
$$

On the one hand, by definition of $A$, since $y=(u, v)$ and $v=\partial_{t} u$, we have

$$
\begin{aligned}
\left(A y, y_{c}\right)_{\mathcal{Y}} & =\left(\nabla \partial_{t} u, \nabla\left(\chi_{c} u\right)\right)_{\mathrm{L}^{2}(\mathcal{D})}-\left(\nabla u, \nabla\left(\chi_{c} \partial_{t} u\right)\right)_{\mathrm{L}^{2}(\mathcal{D})} \\
& \left.=\left(\nabla \partial_{t} u, \nabla\left(\chi_{c} u\right)\right)_{\mathrm{L}^{2}(\mathcal{D})}-\left(\nabla u,\left(\nabla \chi_{c}\right) \partial_{t} u\right)\right)_{\mathrm{L}^{2}(\mathcal{D})}-\left(\nabla u, \chi_{c} \nabla \partial_{t} u\right)_{\mathrm{L}^{2}(\mathcal{D})} \\
& \left.=\left(\nabla \partial_{t} u, \nabla\left(\chi_{c} u\right)\right)_{\mathrm{L}^{2}(\mathcal{D})}-\left(\nabla u,\left(\nabla \chi_{c}\right) \partial_{t} u\right)\right)_{\mathrm{L}^{2}(\mathcal{D})}-\frac{1}{2} \frac{\mathrm{d}}{\mathrm{d} t}\left(\nabla u, \chi_{c} \nabla u\right)_{\mathrm{L}^{2}(\mathcal{D})}
\end{aligned}
$$

On the other hand, we have

$$
\begin{aligned}
\left(\dot{y}, \chi_{c} y\right)_{\mathcal{Y}} & =\left(\nabla \partial_{t} u, \nabla\left(\chi_{c} u\right)\right)_{\mathrm{L}^{2}(\mathcal{D})}+\left(\partial_{t}^{2} u, \chi_{c} \partial_{t} u\right)_{\mathrm{L}^{2}(\mathcal{D})} \\
& =\left(\nabla \partial_{t} u, \nabla\left(\chi_{c} u\right)\right)_{\mathrm{L}^{2}(\mathcal{D})}+\frac{1}{2} \frac{\mathrm{d}}{\mathrm{d} t}\left(\partial_{t} u, \chi_{c} \partial_{t} u\right)_{\mathrm{L}^{2}(\mathcal{D})}
\end{aligned}
$$

We then combine these two results and integrate (42) on $[0, s]$. Benefiting from the fact that $y_{c}(0)=0$ as $y(0)=y_{0}$ is compactly supported in $\mathcal{D}_{0}$, it comes that

$$
\left(\partial_{t} u, \chi_{c} \partial_{t} u\right)_{\mathrm{L}^{2}(\mathcal{D})}(s)+\left(\nabla u, \chi_{c} \nabla u\right)_{\mathrm{L}^{2}(\mathcal{D})}(s)=-2 \int_{0}^{s}\left(\nabla u,\left(\nabla \chi_{c}\right) \partial_{t} u\right)_{\mathrm{L}^{2}(\mathcal{D})}\left(s^{\prime}\right) \mathrm{d} s^{\prime}
$$

Combining a Cauchy-Schwarz inequality and Young's inequality $\left(2|a b| \leq a^{2}+b^{2}\right)$, and using that $\nabla \chi_{c}=0$ in $\mathcal{D} \backslash \mathcal{D}_{c}$, we get

$$
\begin{aligned}
2\left(\nabla u,\left(\nabla \chi_{c}\right) \partial_{t} u\right)_{\mathrm{L}^{2}(\mathcal{D})} & =2\left(\nabla u,\left(\nabla \chi_{c}\right) \partial_{t} u\right)_{\mathrm{L}^{2}\left(\mathcal{D}_{c}\right)} \\
& \leq\|\nabla u\|_{\mathrm{L}^{2}\left(\mathcal{D}_{c}\right)}^{2}+\left\|\left(\nabla \chi_{c}\right) \partial_{t} u\right\|_{\mathrm{L}^{2}\left(\mathcal{D}_{c}\right)}^{2}
\end{aligned}
$$


Since $\chi_{c}$ is smooth, we can bound $\left\|\nabla \chi_{c}\right\|$ by a constant $\kappa>0$, and, by (43) and (44), we get

$$
\begin{aligned}
\left(\partial_{t} u, \chi_{c} \partial_{t} u\right)_{\mathrm{L}^{2}(\mathcal{D})}(s)+\left(\nabla u, \chi_{c} \nabla u\right)_{\mathrm{L}^{2}(\mathcal{D})}(s) & \leq \int_{0}^{s}\|\nabla u\|_{\mathrm{L}^{2}\left(\mathcal{D}_{c}\right)}^{2}\left(s^{\prime}\right)+\kappa\left\|\partial_{t} u\right\|_{\mathrm{L}^{2}\left(\mathcal{D}_{c}\right)}^{2}\left(s^{\prime}\right) \mathrm{d} s^{\prime} \\
& \leq \tilde{\kappa} \int_{0}^{s}\|\nabla u\|_{\mathrm{L}^{2}\left(\mathcal{D}_{c}\right)}^{2}\left(s^{\prime}\right)+\left\|\partial_{t} u\right\|_{\mathrm{L}^{2}\left(\mathcal{D}_{c}\right)}^{2}\left(s^{\prime}\right) \mathrm{d} s^{\prime} \\
& \leq \tilde{\kappa} \int_{0}^{s}\left\|\left.y\left(s^{\prime}\right)\right|_{\mathcal{D}_{c}}\right\|_{\mathcal{Y}}^{2} \mathrm{~d} s^{\prime},
\end{aligned}
$$

where $\tilde{\kappa}=\max (1, \kappa)$. To conclude the proof, we simply need to remark that

$$
\left(\partial_{t} u, \chi_{c} \partial_{t} u\right)_{\mathrm{L}^{2}(\mathcal{D})}(s)+\left(\nabla u, \chi_{c} \nabla u\right)_{\mathrm{L}^{2}(\mathcal{D})}(s) \geq\|y(s)\|_{\mathcal{Y}_{\mathrm{ext}}}^{2},
$$

and to integrate $(45)$ on $[0, T]$.

Lemma 3.8. There exists $T>0$ and $\kappa_{2}>0$ such that for any initial state $y_{0} \in \mathcal{Y}_{0}$

$$
\int_{0}^{T}\left\|C e^{A s} y_{0}\right\|_{\mathcal{Z}}^{2} \mathrm{~d} s \geq \kappa_{2} \int_{0}^{T}\left\|e^{A s} y_{0}\right\|_{\mathcal{Y}_{c}}^{2} \mathrm{~d} s
$$

Proof. We apply the result of equirepartition proved in Appendix A (the proof relies on the use of specific multipliers in (42) to obtain (46), namely $y_{c}=\left(0, \xi_{c} u\right)$, then $\left.y_{c}=\left(\xi_{c}^{2} u, \xi_{c}^{2} v\right)\right)$. We obtain that, for every $T>0$ there exists $\kappa$ such that

$$
\int_{0}^{T}\|\nabla u(t)\|_{\mathrm{L}^{2}\left(\mathcal{D}_{c}\right)}^{2} \mathrm{~d} t \leq \kappa \int_{0}^{T}\left\|\partial_{t} u(t)\right\|_{\mathrm{L}^{2}\left(\mathcal{D}_{\text {obs }}\right)}^{2} \mathrm{~d} t .
$$

Finally, equation (46) is obtained by observing

$$
\left\|e^{A s} y_{0}\right\|_{\mathcal{Y}_{c}}^{2}=\|\nabla u(t)\|_{\mathrm{L}^{2}\left(\mathcal{D}_{c}\right)}^{2}+\left\|\partial_{t} u(t)\right\|_{\mathrm{L}^{2}\left(\mathcal{D}_{c}\right)}^{2} \text { and }\left\|C e^{A s} y_{0}\right\|_{\mathcal{Z}}^{2}=\left\|\partial_{t} u(t)\right\|_{\mathrm{L}^{2}\left(\mathcal{D}_{\mathrm{obs}}\right)}^{2} .
$$

To conclude this section, we thus have proved that the back-and-forth algorithm introduced by [49] can be extended to unbounded domain configurations and restricted to a subclass of initial condition whose support allows the information to propagates into the observation domain. This last restriction reformulates the condition observability result obtained in [31]. In other words, we have shown that in our case the only geometric control condition of interest should be of the form

$$
\forall \xi \in \overline{\mathcal{D}_{0}}, \exists s \in(0, T), \exists \delta>0, B\left(x_{\xi}(s), \delta\right) \subset \mathcal{D}_{\text {obs }},
$$

with $s \mapsto x_{\xi}(s)$ parametrizing the optic rays. The rest of the article will now be devoted to showing how this result can be exploited for practical state reconstructions.

\section{Reformulation in BOUnded DOMAin}

The presented reconstruction method needs now to be discretized to offer a practical algorithm of reconstruction. This step presents, however, numerous challenges and theoretical difficulties to maintain the convergence properties of the implemented algorithm. The first difficulty is to bound the computation domain, typically with transparent boundary conditions or approximated boundary conditions. We thus need to adapt our back-and-forth observer to such bounded formulations and ensure that the boundary conditions do not alter the convergence. This study of the "commutativity" of the transparent boundary conditions treatment and the feedback control definition is rather original, hence we devote a detailed treatment to it. It will have to be complemented by a numerical discretization step which is also challenging (See Section 6). 


\subsection{Example of a bounded domain reformulation in $1 \mathrm{D}$}

In order to present in an illustrative manner the difficulties related to the introduction of adequate artificial boundary conditions and their impact on the back-and-forth observer formulation, let us formally set up the problem in 1D.

We assume that the target function is solution of a problem set in the unbounded domain $\mathbb{R}^{+}$and reads

$$
\mid \begin{array}{lr}
\partial_{t}^{2} u(x, t)-\partial_{x}^{2} u(x, t)=0 & (x, t) \in(0,+\infty) \times[0, T], \\
u(0, t)=0 & t \in[0, T], \\
\left(u, \partial_{t} u\right)(x, 0)=\left(u_{0}(x), v_{0}(x)\right) & x \in(0,1) .
\end{array}
$$

It is well known that this model can be reformulated in the bounded domain $(0,1)$ using a transparent boundary condition. Therefore, solving $(47)$ in the subdomain $(0,1)$ is equivalent to solving

$$
\begin{array}{|lr}
\partial_{t}^{2} u(x, t)-\partial_{x}^{2} u(x, t)=0 & (x, t) \in(0,1) \times[0, T] \\
u(0, t)=0 & t \in[0, T] \\
\partial_{x} u(1, t)+\partial_{t} u(1, t)=0 & t \in[0, T] \\
\left(u, \partial_{t} u\right)(x, 0)=\left(u_{0}(x), v_{0}(x)\right) & x \in(0,1) .
\end{array}
$$

Our objective is now to define boundary conditions for the back-and-forth observers. First, let us remark that our assumption on the observation domain allows in $1 \mathrm{D}$ the choice $\mathcal{D}_{\mathrm{obs}}=(a, 1)$. Being given a target solution $\check{u}$ of (47), we define the forward observer $\hat{u}$ as the solution of

$$
\mid \begin{array}{lr}
\partial_{t}^{2} \hat{u}(x, t)-\partial_{x}^{2} \hat{u}(x, t)+\gamma \mathbb{1}_{(a, 1)}(x) \partial_{t}(\hat{u}(x, t)-\check{u}(x, t))=0 & (x, t) \in(0,+\infty) \times[0, T], \\
\hat{u}(0, t)=0 & t \in[0, T], \\
\left(\hat{u}, \partial_{t} \hat{u}\right)(x, 0)=\left(u_{0}(x), v_{0}(x)\right)+\left(\tilde{u}_{0}(x), \tilde{v}_{0}(x)\right) & x \in(0,1) .
\end{array}
$$

It also satisfies the bounded domain formulation

$$
\begin{array}{|lr}
\partial_{t}^{2} \hat{u}(x, t)-\partial_{x}^{2} \hat{u}(x, t)+\gamma \mathbb{1}_{(a, 1)}(x) \partial_{t}(\hat{u}(x, t)-\check{u}(x, t))=0 & (x, t) \in(0,1) \times[0, T], \\
\hat{u}(0, t)=0 & t \in[0, T], \\
\partial_{x} \hat{u}(1, t)+\partial_{t} \hat{u}(1, t)=0 & t \in[0, T], \\
\left(\hat{u}, \partial_{t} \hat{u}\right)(x, 0)=\left(u_{0}(x), v_{0}(x)\right)+\left(\tilde{u}_{0}(x), \tilde{v}_{0}(x)\right) & x \in(0,1) .
\end{array}
$$

Defining the backward observer in bounded domain is not as obvious. Indeed, the definition of a transparent boundary condition is usually done with an interior initial condition which is not the case of our backward observer. To find the adequate boundary condition, we first deduce from (10) the equations satisfied by the backward observer $\hat{u}_{b}$ in unbounded domain

$$
\mid \begin{array}{lr}
\partial_{t}^{2} \hat{u}_{b}(x, t)-\partial_{x}^{2} \hat{u}_{b}(x, t)-\gamma \mathbb{1}_{(a, 1)}(x) \partial_{t}\left(\hat{u}_{b}(x, t)-\check{u}(x, t)\right)=0 & (x, t) \in(0,+\infty) \times[0, T], \\
\hat{u}_{b}(0, t)=0 & t \in[0, T], \\
\left(\hat{u}_{b}, \partial_{t} \hat{u}_{b}\right)(x, T)=\left(\hat{u}, \partial_{t} \hat{u}\right)(x, T) & x \in(0,1) .
\end{array}
$$

We now introduce the quantity

$$
q(t)=\frac{1}{2}\left(\hat{u}_{b}(t)-\hat{u}(t)\right)
$$

that we will call the adjoint variable for reasons explained in the next section. Subtracting (49) from (51) and dividing by 2 , we get for all $(x, t) \in(0,+\infty) \times[0, T]$

$$
\partial_{t}^{2} q(x, t)-\partial_{x}^{2} q(x, t)-\gamma \mathbb{1}_{(a, 1)}(x) \partial_{t} q(x, t)=-\gamma \mathbb{1}_{(a, 1)}(x)\left(\partial_{t} \check{u}(x, t)-\partial_{t} \hat{u}(x, t)\right) .
$$


Moreover $\left(q, \partial_{t} q\right)(T, x)=(0,0)$ and therefore, $q(t)$ is solution of a wave equation (reversed in time) with localized damping and source term in $(a, 1)$. As a consequence, an exact transparent boundary condition for this problem is given by

$$
\partial_{x} q(1, t)-\partial_{t} q(1, t)=0, \quad t \in[0, T] .
$$

Coming back to the definition of $\hat{u}_{b}$ from $\hat{u}$ and $q$ given by (52), we get the backward observer dynamics rewritten in bounded domain, namely

$$
\mid \begin{array}{lr}
\partial_{t}^{2} \hat{u}_{b}(t, x)-\partial_{x}^{2} \hat{u}_{b}(t, x)+\gamma \mathbb{1}_{(a, 1)}(x)\left(z(t, x)-\partial_{t} \hat{u}_{b}(t, x)\right)=0 & (x, t) \in[0, T] \times(0,1), \\
\partial_{x} \hat{u}_{b}(1, t)-\partial_{t} \hat{u}_{b}(1, t)=-2 \partial_{t} \hat{u}(1, t) & t \in[0, T], \\
\left(\hat{u}_{b}, \partial_{t} \hat{u}_{b}\right)(T, x)=\left(\hat{u}, \partial_{t} \hat{u}\right)(T, x) & x \in(0,1) .
\end{array}
$$

Note that (54) should be understood as a time-reversal formulation, hence the change of sign before all "dissipative" terms. Then the function $\left(\hat{u}_{b}(0, x), \partial_{t} \hat{u}_{b}(0, x)\right)$ is expected to be some approximation of the initial data $\left(u_{0}(x), v_{0}(x)\right)$. We use the projection defined equation (13) to obtain an approximation of $\left(u_{0}(x), v_{0}(x)\right)$ that is compactly supported (here it is required that the support of the inital data $\mathcal{D}_{0}$ satisfies $\mathcal{D}_{0} \subset(0, b)$ with $\left.b<1\right)$ after what the construction of the forward and backward obervers can be re-iterated.

This 1D example shows that the quantity allowing to define unambiguously a backward dynamics compatible with the transparent boundary conditions is not $\hat{u}_{b}$ but $q$. Then eventually, $\hat{u}_{b}$ can be recovered as a byproduct of $\hat{u}$ and $q$. This understanding will be generalized to very general configurations in the next section.

\subsection{An abstract framework}

\subsubsection{The forward dynamics}

The use of transparent boundary conditions requires the introduction of an operator that acts on the boundary of the domain (at least for local-in-space transparent boundary condition such as in the 1D setting). Such operators should add dissipation to the dynamics hence, the direct dynamics is no longer generated by a skew-adjoint operator and we need to modify the abstract setting introduced in Section 2.1. We now introduce the state-space $\mathcal{Y}^{b}$, a Hilbert space associated with function defined on the bounded interior domain $\mathcal{D}_{\text {int }}$ only. On $\mathcal{Y}^{b}$, we consider a maximal dissipative operator - but not necessarily a skew adjoint operator $-A^{b}: D\left(A^{b}\right) \rightarrow \mathcal{Y}^{b}$ with domain $D\left(A^{b}\right)$ dense in $\mathcal{Y}^{b}$ and we consider solutions of the homogeneous system

$$
\left(\mathrm{D}^{b}\right): \mid \begin{aligned}
& \dot{y}=A^{b} y, \quad \text { in } \quad[0, T] \\
& y(0)=y_{0}
\end{aligned}
$$

where $y_{0}$ is an initial data. By the Hille-Yosida theorem, $A^{b}$ generates a strongly continuous semi-group of contraction and the dynamics (55) admits one and only one solution for $y_{0} \in \mathcal{Y}$. Indeed, when considering the more general inhomogeneous case

$$
\mid \begin{aligned}
& \dot{y}=A^{b} y+f, \quad \text { in } \quad[0, T] \\
& y(0)=y_{0}
\end{aligned}
$$

we recall the following standard existence/uniqueness/regularity results for the dynamics (56) directly taken from [7, Chap. 1, Prop. 3.1, 3.3 and Remark 3.5]:

- If $y_{0} \in \mathcal{Y}^{b}$ and $f \in \mathrm{L}^{p}\left(0, T ; \mathcal{Y}^{b}\right)$, the problem (56) has a unique mild - namely in the sense of Duhamel formula - solution $y$ that satisfies

$$
y \in \mathrm{C}^{0}\left([0, T] ; \mathcal{Y}^{b}\right)
$$

- If $y_{0} \in D\left(A^{b}\right)$ and $f \in \mathrm{W}^{1, p}\left(0, T ; \mathcal{Y}^{b}\right)$, the problem (56) has a unique strict solution $y$ that satisfies

$$
y \in \mathrm{C}^{1}\left([0, T] ; \mathcal{Y}^{b}\right) \cap \mathrm{C}^{0}\left([0, T] ; D\left(A^{b}\right)\right) .
$$




\subsubsection{The adjoint dynamics}

We denote $A^{\text {b* }}$ the adjoint of $A^{b}$, which by definition satisfies

$$
\forall(y, q) \in D\left(A^{b}\right) \times D\left(A^{b *}\right), \quad\left(A^{b} y, q\right)_{\mathcal{Y}}^{b}=\left(y, A^{b *} q\right)_{\mathcal{Y}}^{b} .
$$

By the Lumer and Phillips theorem, $A^{\text {b* }}$ is also maximal dissipative (see for instance [7, Chap. 1, Theorem $2.8]$ ) and generates a strongly continuous semi-group of contraction. Therefore, we have $A^{b *}: D\left(A^{b *}\right) \rightarrow \mathcal{Y}^{b}$ with domain $D\left(A^{b *}\right)$ dense in $\mathcal{Y}^{b}$. Hence, we can define solution of adjoint dynamics

$$
\mid \begin{aligned}
& \dot{q}+A^{b *} q=f, \quad \text { in } \quad[0, T] \\
& q(T)=q_{T}
\end{aligned}
$$

where $q_{T}$ is a given final state and $f$ a source term whose regularity will be specified in the next paragraph. Note that the problem is solved backward in time hence the "change of sign" in the use of $A^{\text {b* }}$. More precisely, we have for (57), again from [7, Chap. 1, Prop. 3.1, 3.3 and Remark 3.5]:

- If $q_{T} \in \mathcal{Y}^{b}$ and $f \in \mathrm{L}^{p}\left(0, T ; \mathcal{Y}^{b}\right)$, the problem (57) has a unique mild solution $q$ that satisfies

$$
q \in \mathrm{C}^{0}\left([0, T] ; \mathcal{Y}^{b}\right)
$$

- If $q_{T} \in D\left(A^{b *}\right)$ and $f \in \mathrm{W}^{1, p}\left(0, T ; \mathcal{Y}^{b}\right)$, the problem (57) has a unique strict solution $q$ that satisfies

$$
q \in \mathrm{C}^{1}\left([0, T] ; \mathcal{Y}^{b}\right) \cap \mathrm{C}^{0}\left([0, T] ; D\left(A^{b *}\right)\right) .
$$

\subsection{The abstract back-and-forth algorithm}

\subsubsection{The forward observer}

As in Section 2.2, we define the forward observer from a guess on the initial data as the solution of

$$
\left(\mathrm{F}^{b}\right): \mid \begin{aligned}
& \dot{\hat{y}}(t)=A^{\mathrm{b}} \hat{y}+\gamma C^{b *}\left(z-C^{b} \hat{y}\right)=A_{\gamma}^{b} \hat{y}+\gamma C^{b *} z, \quad \text { in } \quad[0, T], \\
& \hat{y}(0)=\hat{y}_{0},
\end{aligned}
$$

with $z(t)$ some (possibly noisy) observation of the target dynamic $\check{y}(t)$ that belongs to the same Hilbert space $\mathcal{Z}$ as introduced in Section 2.1 and where

$$
C^{b} \in \mathcal{L}\left(\mathcal{Y}^{b}, \mathcal{Z}\right), \quad A_{\gamma}^{b}=A^{b}-\gamma C^{b *} C^{b} .
$$

As done in Section 2.2, the operator $C^{\text {b* }}$ is defined to belong to $\mathcal{L}\left(\mathcal{Z}, \mathcal{Y}^{b}\right)$, and without loss of generality we assume that

$$
\left\|C^{b}\right\|_{\mathcal{L}\left(\mathcal{Y}^{b}, \mathcal{Z}\right)} \leq 1
$$

Following [35, Remark 2.2], it can be easily shown that the operator $A_{\gamma}^{b}$ with $D\left(A_{\gamma}^{b}\right) \equiv D\left(A^{b}\right)$ is the generator of a strongly continuous semi-group of contraction using the same ideas exposed at the beginning of Section 4.2 or by perturbation techniques (see [46, Chapter 3]). Note that the sign of the perturbation does matter here, since it qualifies a dissipative perturbation. Therefore, if $\hat{y}_{0}$ belong to $\mathcal{Y}^{b}$ and $z$ belongs to $\mathrm{L}^{1}(0, T ; \mathcal{Z})$, then there exists a unique mild solution of $(58)$.

\subsubsection{The adjoint state}

Following the construction of Section 4.1, we now define the adjoint dynamics. By inspection of (53a) we set

$$
\left(\operatorname{Ad}^{b}\right): \mid \begin{aligned}
& \dot{q}+A_{\gamma}^{b *} q=-\gamma C^{b *}\left(z-C^{b} \hat{y}\right), \quad \text { in }[0, T] \\
& q(T)=0
\end{aligned}
$$


with, since $C^{b *} C^{b}$ is a bounded linear operator of $\mathcal{Y}^{b}$ into $\mathcal{Y}^{b}$,

$$
A_{\gamma}^{b *}=A^{b *}-\gamma C^{b *} C^{b}
$$

Since $\hat{u} \in \mathrm{C}^{0}\left([0, T] ; \mathcal{Y}^{b}\right), z \in \mathrm{L}^{1}\left(0, T ; \mathrm{L}^{2}(\mathcal{Z})\right)$ and because of the definition of $C^{b}$ the term $\gamma C^{b *}\left(z-C^{b} \hat{y}\right)$ can be seen as a source term in $\mathrm{L}^{1}\left(0, T ; \mathcal{Y}^{b}\right)$, hence, the adjoint dynamics (59) is always well defined backward in time as soon as the direct problem is well defined forward in time. This is the case here as $A_{\gamma}^{b *}$ generates a strongly continuous semi-group of contraction. Therefore, there exists a unique mild solution of (59).

Remark 4.1. The dynamics (59) can be interpreted as the Lagrange multiplier associated with the minimization of the functional

$$
\mathcal{J}(\hat{y}(0))=\frac{1}{2} \int_{0}^{T}\left\|z-C^{b} \hat{y}\right\|_{\mathcal{Z}}^{2} \mathrm{~d} s
$$

under the constraint that $\hat{y}$ is solution of the forward observer with initial data $\hat{y}(0)$. This remark justifies that we call $q$ the adjoint state.

\subsubsection{The backward observer}

Our objective is to give an abstract formulation of the backward observer in bounded domain. By definition of the adjoint observer in Section 4.1, we define

$$
\forall t \in[0, T], \quad \hat{y}_{b}(t)=\hat{y}(t)+2 q(t) .
$$

As a difference of mild solutions, $\hat{y}_{b}$ is well defined as a function in $\mathrm{C}^{0}\left([0, T] ; \mathcal{Y}^{b}\right)$. However, if we want to further characterize the dynamics of $\hat{y}_{b}$, we need to assume that $D\left(A^{b *}\right) \equiv D\left(A^{b}\right)$. Therefore assuming that $\hat{y}(t)$ and $q(t)$ are strict solutions we can compute

$$
\begin{aligned}
\dot{\hat{y}}_{b}=\dot{\hat{y}}+2 \dot{q} & =A_{\gamma}^{b} \hat{y}+\gamma C^{b *} z-2 A_{\gamma}^{b *} q-2 \gamma C^{b *}\left(z-C^{b} \hat{y}\right) \\
& =A^{b} \hat{y}-2 A^{b *} q-\gamma C^{b *}\left(z-C^{b} \hat{y}_{b}\right) .
\end{aligned}
$$

Then, as a consequence of $\hat{y}_{b} \in \mathrm{C}^{1}\left([0, T] ; \mathcal{Y}^{b}\right) \cap \mathrm{C}^{0}\left([0, T] ; D\left(A^{b}\right)\right)$, we see that

$$
A^{b} \hat{y}-2 A^{b *} q=-A^{b *} \hat{y}_{b}+\left(A^{b}+A^{b *}\right) \hat{y}
$$

and finally equation (61) can be understood in $\mathrm{C}^{0}\left(0, T ; \mathcal{Y}^{b}\right)$ and reads

$$
\dot{\hat{y}}_{b}=-A^{b *} \hat{y}_{b}-\gamma C^{b *}\left(z-C^{b} \hat{y}_{b}\right)+\left(A^{b}+A^{b *}\right) \hat{y}
$$

giving finally, when $D\left(A^{\text {b* }}\right) \equiv D\left(A^{b}\right)$,

$$
\mid \begin{aligned}
& \dot{\hat{y}}_{b}=-A^{b *} \hat{y}_{b}-\gamma C^{b *}\left(z-C^{b} \hat{y}_{b}\right)+B B^{*} \hat{y} \\
& \hat{y}_{b}(T)=\hat{y}(T),
\end{aligned}
$$

where the term $\left(A^{b}+A^{b *}\right) \hat{y}=B B^{*} \hat{y}$ is a boundary source term in the backward observer, typically encountered in time reversal approaches, see for instance [16] and references therein. However, as it is not true in general that $D\left(A^{\mathrm{b} *}\right)=D\left(A^{\mathrm{b}}\right)$, we will avoid using the backward dynamics (63) and instead, we will only rely on the sufficient, and in fact more general, definition of $q(t)$.

\subsubsection{Reconstruction-error operator}

We can now deduce the expression of the operator that maps $\hat{y}(0)$ to $\hat{y}_{b}(0)=\hat{y}(0)+2 q(0)$ using the observation at hand (this operator is denoted by $\Lambda_{\gamma}^{b}$ ). To simplify the presentation, we first consider a perfect 
measurement configuration, more precisely $z(t)=C^{b} \check{y}(t)$ with $\check{y}(t)$ solution of (55), i.e. the dynamics (56) without source term. Following Proposition 3.1, we introduce

$$
\tilde{y}(t)=\hat{y}(t)-\check{y}(t) \quad \text { and } \quad \tilde{y}_{b}(t)=\tilde{y}(t)+2 q(t)\left(=\hat{y}_{b}(t)-\check{y}(t)\right),
$$

and define $\Lambda_{\gamma}^{b}$ as the reconstruction-error operator, namely $\tilde{y}_{b}(0)=\Lambda_{\gamma}^{b} \tilde{y}(0)$.

Proposition 4.2. The operator $\Lambda_{\gamma}^{b}$ is a bounded positive self-adjoint operator from $\mathcal{Y}^{b}$ to $\mathcal{Y}^{b}$ defined by

$$
\Lambda_{\gamma}^{b}=\mathbb{1}-2 \gamma \int_{0}^{T} e^{A_{\gamma}^{b *} s} C^{b *} C^{b} e^{A_{\gamma}^{b} s} \mathrm{~d} s
$$

moreover, $\left\|\Lambda_{\gamma}^{b}\right\|_{\mathcal{L}\left(\mathcal{Y}^{b}, \mathcal{Y}^{b}\right)} \leq 1$.

Proof. The error $\tilde{y}$ and the adjoint $q$ satisfy

$$
\dot{\tilde{y}}=A_{\gamma}^{b} \tilde{y}, \quad \dot{q}+A_{\gamma}^{b *} q=\gamma C^{b *} C^{b} \tilde{y}(t), \quad \text { in }[0, T] .
$$

Therefore we have $\tilde{y}(t)=e^{A_{\gamma}^{b} t} \tilde{y}_{0}$ and by definition of $\tilde{y}_{b}(t)$ and $q(t)$ we get that

$$
\dot{\tilde{y}}_{b}(t)=\tilde{y}(t)-2 \gamma \int_{t}^{T} e^{A_{\gamma}^{b *}(s-t)} C^{b *} C^{b} \tilde{y}(s) \mathrm{d} s .
$$

The characterization (64) of $\Lambda_{\gamma}^{b}$ is obtained by setting $t=0$. Therefore, $\Lambda_{\gamma}^{b}$ is clearly self-adjoint. To now prove the positiveness, we proceed by energy analysis using the maximal dissipativity property of the operators. First, let us assume that the initial data $\tilde{y}_{0}$ is sufficiently smooth so that $\tilde{y}(t)$ is a strict solution, i.e., $\tilde{y}_{0} \in D\left(A_{\gamma}^{b}\right)$. Then, by taking the scalar product of the first equation of (65) with $\tilde{y}(t)$, we have

$$
\frac{1}{2} \frac{d}{d t}\|\tilde{y}\|_{\mathcal{Y}^{b}}^{2}+\gamma\left\|C^{b} \tilde{y}\right\|_{\mathcal{Z}}^{2} \leq 0 \quad \Rightarrow \quad 2 \gamma \int_{0}^{T}\left\|C^{b} \tilde{y}(t)\right\|_{\mathcal{Z}}^{2} \mathrm{~d} t \leq\left\|\tilde{y}_{0}\right\|_{\mathcal{Y}^{b}}^{2}
$$

which leads to

$$
2 \gamma \int_{0}^{T}\left(e^{A_{\gamma}^{b *} s} C^{b *} C^{b} e^{A_{\gamma}^{b} s} \tilde{y}_{0}, \tilde{y}_{0}\right)_{\mathcal{Y}^{b}} \mathrm{~d} s \leq\left\|\tilde{y}_{0}\right\|_{\mathcal{Y}^{b}}^{2} \quad \Rightarrow \quad 0 \leq\left(\Lambda_{\gamma}^{b} \tilde{y}_{0}, \tilde{y}_{0}\right)_{\mathcal{Y}^{b}} \leq\left\|\tilde{y}_{0}\right\|_{\mathcal{Y}^{b}}^{2}
$$

Finally by density of $D\left(A_{\gamma}^{b}\right)$ in $\mathcal{Y}^{b}$, we have $\Lambda_{\gamma}^{b}$ positive and bounded in $\mathcal{L}\left(\mathcal{Y}^{b}, \mathcal{Y}^{b}\right)$ and $\left\|\Lambda_{\gamma}^{b}\right\|_{\mathcal{L}\left(\mathcal{Y}^{b}, \mathcal{Y}^{b}\right)} \leq 1$.

4.3.5. Iterative algorithm and convergence analysis

We now proceed to the convergence analysis of the noise-free case. Being given a target solution $\check{y}(t)$ of (55), our objective is to recover the initial data $\check{y}(0)=y_{0}$. To follow our unbounded approach developed in Section 2.2, we introduce a closed subspace

$$
\mathcal{Y}_{0}^{b} \subset \mathcal{Y}^{b}
$$

and the orthogonal projector $\Pi^{b}$ on this subspace. Then, the back-and-forth algorithm is a direct extension of the algorithm presented in Section 2.2, except that the adjoint dynamics is used instead of the backward observer. It reads:

Assume $\hat{y}(0)=\hat{y}_{0} \in \mathcal{Y}_{0}^{b}$ given, compute for $n \geq 0$

$$
\left(\text { Ad }^{b} \& \mathrm{~F}^{b}+\text { Proj }\right): \mid \begin{aligned}
& \dot{\hat{y}}^{(n)}=A_{0}^{b} \hat{y}^{(n)}+\gamma C^{\mathrm{b} *}\left(z-C^{\mathrm{b}} \hat{y}^{(n)}\right), \quad \text { in }[0, T], \\
& \hat{y}^{(n)}(0)=\Pi^{\mathrm{b}}\left(\hat{y}^{(n-1)}-2 q^{(n-1)}\right)(0), \\
& \dot{q}^{(n)}+A_{\gamma}^{b *} q^{(n)}=-\gamma C^{b *}\left(z-C^{b} \hat{y}^{(n)}\right), \quad \text { in }[0, T], \\
& q^{(n)}(T)=0,
\end{aligned}
$$


where $\Pi^{b}\left(\hat{y}^{(-1)}+2 q^{(-1)}\right)(0)=\hat{y}(0)=\check{y}_{0}+\tilde{y}(0) \in \mathcal{Y}_{0}^{b}$.

As for the unbounded space case, one can see that, if $z(t)=C \check{y}(t)$, then the reconstruction error $\tilde{y}^{n}(0)$ satisfies

$$
\tilde{y}^{n}(0)=\left(\Pi^{b} \Lambda_{\gamma}^{b} \Pi^{b}\right)^{n} \tilde{y}(0) .
$$

Therefore, the algorithm converges exponentially fast if the norm of $\Pi^{b} \Lambda_{\gamma}^{b} \Pi^{b}$ is strictly smaller than one. This is the object of the following condition and lemma.

Criterion 4.3 (Observability condition in bounded domain). There exists $T>0$ and $\kappa$ such that, for all $y_{0} \in \mathcal{Y}_{0}^{b}$, we have

$$
\int_{0}^{T}\left\|C^{b} e^{A^{b} t} y_{0}\right\|_{\mathcal{Z}}^{2} \mathrm{~d} t \geq \kappa\left\|y_{0}\right\|_{\mathcal{Y}^{b}}^{2} .
$$

Lemma 4.4. The operator $\Pi^{b} \Lambda_{\gamma}^{b} \Pi^{b}$ is bounded, symmetric and positive. Moreover, if the Observability Condition 4.3 is satisfied then, $\left\|\Pi^{\mathrm{b}} \Lambda_{\gamma}^{b} \Pi^{\mathrm{b}}\right\|_{\mathcal{L}\left(\mathcal{Y}^{b}, \mathcal{Y}^{b}\right)}<1$ as soon as $\gamma>0$.

Proof. We proceed as in Proposition 3.2. We only need to prove that there exists $T>0$ and $\tilde{\kappa}$ such that, for all $y_{0} \in \mathcal{Y}_{0}^{b}$

$$
\int_{0}^{T}\left\|C^{b} e^{A_{\gamma}^{b} s} y_{0}\right\|_{\mathcal{Z}}^{2} \mathrm{~d} s \geq \tilde{\kappa}\left\|y_{0}\right\|_{\mathcal{Y}^{b}}^{2}
$$

This result is a consequence of the admissibility and controllability assumptions in the theorem statement and is proven below. Assume first that $y_{0}$ belongs to $D\left(A^{b}\right) \cap \mathcal{Y}_{0}^{b}$ (we recall that $D\left(A^{b}\right)=D\left(A_{\gamma}^{b}\right)$ ). We introduce the error $\tilde{\tilde{y}}$ defined by

$$
\tilde{\tilde{y}}=\left(e^{A^{\mathrm{b}} t}-e^{A_{\gamma}^{\mathrm{b}} t}\right) y_{0} \quad \in \quad \mathrm{C}^{1}\left([0, T] ; \mathcal{Y}^{\mathrm{b}}\right) \cap \mathrm{C}^{0}\left([0, T] ; D\left(A^{\mathrm{b}}\right)\right),
$$

which satisfies

$$
\dot{\tilde{\tilde{y}}}=A^{b} \tilde{\tilde{y}}+\gamma C^{b *} C^{b} e^{A_{\gamma}^{b} t} y_{0}, \quad \tilde{\tilde{y}}(0)=0 .
$$

By standard energy analysis, since $A^{\text {b }}$ is maximal dissipative, one can show that

$$
\frac{1}{2}\|\tilde{\tilde{y}}(t)\|_{\mathcal{Y}^{b}}^{2} \leq \gamma \int_{0}^{t}\left\|C^{b} e^{A_{\gamma}^{b} s} y_{0}\right\|_{\mathcal{Z}}\left\|C^{b} \tilde{\tilde{y}}(s)\right\|_{\mathcal{Z}} \mathrm{d} s .
$$

Using Young's inequality $2 a b \leq a^{2} / \nu^{2}+b^{2} \nu^{2}$ with $0<\nu<1$, we get

$$
\|\tilde{\tilde{y}}(t)\|_{\mathcal{Y}^{b}}^{2}-\gamma \nu^{2} \int_{0}^{t}\left\|C^{b} \tilde{\tilde{y}}(s)\right\|_{\mathcal{Z}}^{2} \mathrm{~d} s \leq \frac{\gamma}{\nu^{2}} \int_{0}^{t}\left\|C^{b} e^{A_{\gamma}^{b} s} y_{0}\right\|_{\mathcal{Z}}^{2} \mathrm{~d} s .
$$

Integrating over $t \in[0, T]$ and choosing $\nu$ sufficiently small we can show that

$$
\int_{0}^{T}\|\tilde{\tilde{y}}(t)\|_{\mathcal{Y}^{b}}^{2} \mathrm{~d} t \leq \alpha \int_{0}^{T}\left\|C^{b} e^{A_{\gamma}^{b} t} y_{0}\right\|_{\mathcal{Z}}^{2} \mathrm{~d} t
$$

where $\alpha$ is some positive constant depending on $\gamma$ and $T$. Moreover, using the observability assumption (for some given $T>0$ ), then the decomposition $e^{A^{b} t} y_{0}=\tilde{\tilde{y}}+e^{A_{\gamma}^{b} t} y_{0}$ and $\|a+b\|^{2} \leq 2\|a\|^{2}+2\|b\|^{2}$ we have

$$
\left\|y_{0}\right\|_{\mathcal{Y}^{b}}^{2} \leq \kappa^{-1} \int_{0}^{T}\left\|C^{b} e^{A^{b} t} y_{0}\right\|_{\mathcal{Z}}^{2} \mathrm{~d} t \leq 2 \kappa^{-1} \int_{0}^{T}\left\|C^{b} y_{\gamma}(t)\right\|_{\mathcal{Z}}^{2} \mathrm{~d} t+2 \kappa^{-1} \int_{0}^{T}\left\|C^{b} e^{A_{\gamma}^{b} t} y_{0}\right\|_{\mathcal{Z}}^{2} \mathrm{~d} t .
$$

Finally, using (71) one can see that

$$
\left\|y_{0}\right\|_{\mathcal{Y}^{b}}^{2} \leq 2 \kappa^{-1}(1+\alpha) \int_{0}^{T}\left\|C^{b} e^{A_{\gamma}^{b} t} y_{0}\right\|_{\mathcal{Z}}^{2} \mathrm{~d} t
$$

which is exactly the observability inequality (70) that is required to finish the proof. 


\subsection{Stability analysis}

\subsubsection{Noisy data analysis}

In this section, we now assess the robustness of the bounded domain reformulation of our back-andforth Algorithm (68) with respect to noisy data. We thus denote by $\eta(t) \in \mathcal{Z}$ the difference between the measurements at hand and the measurements that would have been produced by the target trajectory $\check{y}$ solution of (55). More precisely, as in Section 3.2 we assume that

$$
\forall t \in[0, T], \quad z(t)=C^{b} \check{y}(t)+\eta(t)
$$

with again $\eta \in \mathrm{L}^{1}(0, T ; \mathcal{Z})$.

Theorem 4.5. Let assume that the Observability Condition 4.3 holds. Then, considering $\gamma>0$, $z(t)=$ $C^{b} \check{y}(t)+\eta(t)$ with $\check{y}(t)=e^{A^{b} t} \check{y}_{0}, \check{y}_{0} \in \mathcal{Y}_{0}^{b}$ and $\eta \in \mathrm{L}^{1}(0, T ; \mathcal{Z})$, the algorithm (68) converges to the initial state $\check{y}_{0}$ in the sense that there exists $0 \leq \alpha<1$, independent of $\check{y}_{0}$ such that

$$
\left\|\hat{y}^{(n)}(0)-\check{y}_{0}\right\|_{\mathcal{Y}^{\mathrm{b}}} \leq \alpha^{n}\left\|\check{y}_{0}-\hat{y}_{0}\right\|_{\mathcal{Y}^{\mathrm{b}}}+\frac{2 \gamma}{1-\alpha} \int_{0}^{T}\|\eta(s)\|_{\mathcal{Z}} \mathrm{d} s
$$

Proof. Since our algorithm is linear we only need to consider the forward problem $(58)$ with $z(t)=\eta(t)$ and zero initial condition, as well as the corresponding adjoint problem (59) with the same observation $z(t)$. Then we have

$$
\hat{y}(t)=\gamma \int_{0}^{t} e^{A_{\gamma}^{\mathrm{b}}(t-s)} C^{\mathrm{b} *} \eta(s) \mathrm{d} s, \quad q(t)=\gamma \int_{t}^{T} e^{A_{\gamma}^{\mathrm{b} *}(s-t)} C^{\mathrm{b} *}\left(\eta(s)-C^{b} \hat{y}(s)\right) \mathrm{d} s .
$$

If we denote $\hat{y}_{b}(t)=\hat{y}(t)+2 q(t)$, we need to estimate $\hat{y}_{b}(0)=2 q(0)$ since it corresponds to the error - related to noise - on the initial data reconstruction after one forth and back iteration.

$$
\hat{y}_{b}(0)=2 \gamma \int_{0}^{T} e^{A_{\gamma}^{\mathrm{b} *}} C^{\mathrm{b} *} \eta(t) \mathrm{d} t-2 \gamma^{2} \int_{0}^{T} \int_{0}^{t} e^{A_{\gamma}^{\mathrm{b} *} t} C^{b *} C^{b} e^{A_{\gamma}^{b}(t-s)} C^{\mathrm{b} *} \eta(s) \mathrm{d} s \mathrm{~d} t .
$$

Exchanging the last two integrals, we find

$$
\int_{0}^{T} \int_{0}^{t} e^{A_{\gamma}^{b *} t} C^{b *} C^{b} e^{A_{\gamma}^{b}(t-s)} C^{b *} \eta(s) \mathrm{d} s \mathrm{~d} t=\int_{0}^{T} \int_{s}^{T} e^{A_{\gamma}^{b *} t} C^{b *} C^{b} e^{A_{\gamma}^{b}(t-s)} C^{b *} \eta(s) \mathrm{d} t \mathrm{~d} s
$$

As $t-s \geq 0$ in the integrand, we can use the semi-group property to write

$$
e^{A_{\gamma}^{b *} t}=e^{A_{\gamma}^{b *} s} e^{A_{\gamma}^{b *}(t-s)}
$$

and the above quantity is equal to

$$
\int_{0}^{T} e^{A_{\gamma}^{\mathrm{b} *} s} \Upsilon(T-s) C^{b *} \eta(s) \mathrm{d} s \quad \text { with } \quad \Upsilon(T-s)=\int_{0}^{T-s} e^{A_{\gamma}^{\mathrm{b} *} t} C^{\mathrm{b} *} C^{\mathrm{b}} e^{A_{\gamma}^{\mathrm{b}} t} \mathrm{~d} t
$$

where from $(67), 2 \gamma \Upsilon(T) \in \mathcal{L}\left(\mathcal{Y}^{b}, \mathcal{Y}^{b}\right)$ is of norm smaller than 1 for all $T \geq 0$. Finally, we obtain that

$$
\hat{y}_{b}(0)=2 \gamma \int_{0}^{T} e^{A_{\gamma}^{b *} s}(I-\gamma \Upsilon(T-s)) C^{b *} \eta(s) \mathrm{d} s \quad \Rightarrow \quad\left\|\hat{y}_{b}(0)\right\|_{\mathcal{Y}^{b}} \leq 2 \gamma \int_{0}^{T}\|\eta(s)\|_{\mathcal{Z}} \mathrm{d} s .
$$

The rest of the proof is similar to the computations done in Section 3.2 and is omitted. 


\subsubsection{Combining modeling and measurement noise}

We can now consider a more practical configuration involving approximate transparent boundary conditions and noisy measurements. More precisely, we distinguish two types of errors:

- Measurement noise: in practice due to any measurement device acquisition, it here corresponds to a perturbation in the same observation space of the measurements.

- Modeling noise: The use of transparent boundary conditions when proceeding to the model discretization, can be seen as a modeling error in the definition of the underlying continuous model. An important literature exists about choosing adequate transparent boundary conditions for wave-like systems. In some cases, exact transparent boundary conditions can be defined [26, 29] leading to a formulation of our estimation problem in the unbounded domain case (Section 2). However, in numerous configurations, only approximate transparent boundary conditions are available - see for instance [27] - and we need to control their impact on our reconstruction algorithm.

Using approximate boundary conditions and noisy measurements will clearly introduce a consistency error in our reconstruction. However, as shown in the previous paragraph, we are still able to introduce a stable reconstruction-error operator and our reconstruction algorithm will still be convergent in a certain sense. Moreover, the use of the data at hand combined with the stability of the reconstruction-error operator allow to alleviate the impact of the consistency error introduced by the approximate boundary condition as discussed below. To be more accurate, we consider that the data at hand are of the form

$$
z=C^{b} e^{A^{b} t} \check{y}_{0}+\eta_{m}+\eta_{b}
$$

where $C e^{A^{b} t} \check{y}_{0}$ is the noise-free observation of the dynamics with the approximate boundary condition, $\eta_{m}$ is the noise associated with the measurements and $\eta_{b}$ corresponds to discrepancy between the exact transparent boundary condition and the approximate boundary condition. For $\check{y}_{0} \in \mathcal{Y}_{0}^{b}$, we have

$$
\eta_{b}(t)=C \check{y}(t)-C^{b} e^{A^{b} t} \check{y}_{0} \in \mathrm{L}^{1}(0, T ; \mathcal{Z})
$$

where $\check{y}(t)$ is the solution of the target solution of (3) in the unbounded domain configuration. Then, Theorem 4.5 shows how the quality of the reconstruction is deteriorated by the approximation of the transparent boundary condition, i.e. the modeling noise. Indeed, the estimate (73) indicates that:

- the observability inequality (69) is necessary to obtain the exponential convergence, i.e. $\alpha<1$, and a reasonable control of the modeling noise. It means in particular that the final time of observation $T$ should be large enough so that the observability inequality holds, however, it should not be too large since the approximation of transparent boundary condition may not catch accurately long time behaviors of the solution, and its accuracy may deteriorate rapidly over time.

- the operator $A^{b}$ should generate a semi-group which is a good approximation of the semi-group generated by $A$ (the generator of the dynamics in unbounded domain) but only in the observation region and only for initial data in $\mathcal{Y}_{0}^{b}$. This is an important remark since it is well known that standard approximations of transparent boundary conditions are not accurate for waves with grazing incidence and such types of waves may be avoided because of the support of the initial data that is reconstructed.

\section{Application to the Wave equation with approximate transparent BOUNDARY CONDITIONS}

To simplify our presentation, we limit ourselves to first-order transparent boundary conditions approximations as defined in [20], which are local in time. However, we believe that our result extends to more general approximations and could be extended for instance to operators which are non-local in time. Moreover, for non-local approximations that are based on only a finite number of auxiliary unknowns, we have still the luxury of defining a local operator on an augmented system with comparable properties of skewadjointness [45]. In fact, we will show in the present section that even for the simplest case of first order transparent boundary conditions, such augmented states have to be introduced. 


\subsection{Specific formulation in the abstract framework}

The use of transparent boundary conditions requires the introduction of an operator that acts on the domain boundary (at least for local-in-space transparent boundary condition such as in the 1D setting). We here refer to the very complete presentations in [35,52] and some results and notation given in [34] and [33]. We also rely on the definition of the domain done in Section 2.1 and set

$$
\Omega=\mathcal{D}_{\text {int }} \backslash \overline{\mathcal{O}}, \quad \Gamma=\partial \mathcal{D}_{\text {int }} \backslash \partial \mathcal{D}, \quad \Sigma=\partial \mathcal{D} \cap \overline{\mathcal{D}_{\text {int }}}
$$

where $\Gamma$ denotes the boundary on which the approximate transparent boundary condition acts whereas $\Sigma$ is the subdomain of the boundary - included in the interior domain - where the solution should vanish (this includes the obstacle boundary $\partial \mathcal{O}$ ). We introduce the Hilbert spaces $\mathcal{V}$ and $\mathcal{H}$ defined by

$$
\mathcal{V}=\left\{u \in \mathrm{H}^{1}(\Omega), u=0 \text { on } \Sigma\right\}, \quad \mathcal{H}=\mathrm{L}^{2}(\Omega) .
$$

We have the usual triple inclusion $\mathcal{V} \subset \mathcal{H} \equiv \mathcal{H}^{\prime} \subset \mathcal{V}^{\prime}$ and $\mathcal{V}$ is dense in $\mathcal{H}$. We recall the problem: for $\left(u(0), \partial_{t} u(0)\right) \in \mathcal{Y}_{0}^{b}$ given, find $u(t) \in \mathcal{V}$ such that

$$
\mid \begin{array}{lll}
\partial_{t}^{2} u-\Delta u=0 & \text { in } & {[0, T] \times \Omega} \\
\nabla u \cdot n+\delta u+\beta \partial_{t} u=0 & \text { on } & {[0, T] \times \Gamma}
\end{array}
$$

The boundary condition in (75), is dissipative as soon as $\beta>0$, hence this problem is well-posed [27]. When $\delta=0$ and $\beta=1$, the boundary condition is transparent for waves with normal incidence only, which means that in a $1 D$ setting the boundary condition is exact, otherwise $2 \delta$ should be chosen equal to the curvature of the boundary to obtain a first order approximate boundary condition (however, in what follows we assume that $\beta$ and $\gamma$ do not depend on space for the sake of simplicity). We will use $\beta \neq 1$ in Section 6 to numerically investigate the impact of the approximation quality.

\subsubsection{The operator form of the system}

We define the operator $A_{0}^{b}$ in $\mathcal{L}\left(\mathcal{V}, \mathcal{V}^{\prime}\right)$ such that

$$
\forall(u, w) \in \mathcal{V} \times \mathcal{V}, \quad\left\langle A_{0}^{b} u, w\right\rangle_{\mathcal{V}^{\prime}, \mathcal{V}}=(\nabla u, \nabla w)_{\mathrm{L}^{2}\left(\mathcal{D}_{\text {int }}\right)}, \quad D\left(A_{0}^{b}\right)=\left\{u \in \mathcal{V}, A_{0}^{b} u \in \mathcal{H}\right\}
$$

Note that $A_{0}^{b}$ is an operator set in bounded domain with Neumann boundary conditions where the transparent boundary conditions will apply. The operator $A_{0}^{b}$ is self-adjoint coercive and its inverse and fractional power can be defined by interpolation. We have

$$
\forall(u, w) \in \mathcal{V} \times \mathcal{V}, \quad\left\langle A_{0}^{b} u, w\right\rangle_{\mathcal{V}^{\prime}, \mathcal{V}}=\left\langle A_{0}^{b} w, u\right\rangle_{\mathcal{V}^{\prime}, \mathcal{V}}=\left(A_{0}^{b \frac{1}{2}} u, A_{0}^{b^{\frac{1}{2}}} w\right)_{\mathcal{H}}
$$

where $(\cdot, \cdot)_{\mathcal{H}}$ denotes the usual $\mathrm{L}^{2}$ scalar product on $\mathcal{D}_{\text {int }}$. The operator $A_{0}^{b^{\frac{1}{2}}}$ can be seen as an operator in $\mathcal{L}(\mathcal{V}, \mathcal{H})$ and can be extended to $\mathcal{L}\left(D\left(A_{0}^{b}\right), \mathcal{V}\right)$ where $D\left(A_{0}^{b}\right)$ is endowed with the graph norm of $A_{0}^{b}$. As in [7], we write

$$
A_{0}^{\frac{1}{2}} \in \mathcal{L}(\mathcal{V}, \mathcal{H}) \cap \mathcal{L}\left(D\left(A_{0}^{b}\right), \mathcal{V}\right)
$$

Moreover, we equip $\mathcal{V}$ with the scalar product

$$
(u, w)_{\mathcal{V}}=\left(A_{0}^{\mathrm{b} \frac{1}{2}} u, A_{0}^{\mathrm{b} \frac{1}{2}} w\right)_{\mathcal{H}},
$$

since $(\nabla \cdot \nabla \cdot)_{\mathrm{L}^{2}\left(\mathcal{D}_{\text {int }}\right)}$ is coercive on $\mathcal{V}$. Finally, the inverse operator $\left(A_{0}^{b}\right)^{-\frac{1}{2}}$ is well defined, belongs to $\mathcal{L}\left(\mathcal{V}^{\prime}, \mathcal{H}\right)$, and can be extended to $\mathcal{L}(\mathcal{H}, \mathcal{V})$, i.e.

$$
\left(A_{0}^{b}\right)^{-\frac{1}{2}} \in \mathcal{L}\left(\mathcal{V}^{\prime}, \mathcal{H}\right) \cap \mathcal{L}(\mathcal{H}, \mathcal{V})
$$


Now we introduce the operator $N \in \mathcal{L}\left(H^{-\frac{1}{2}}(\Gamma), \mathcal{V}\right)$ defined as

$$
N: \mid \begin{array}{rlrl}
H^{-\frac{1}{2}}(\Gamma) \rightarrow \mathcal{V} & \\
g u u \text { s.t. } & =0, & & \text { in } \mathcal{D}_{\mathrm{int}}, \\
\nabla u \cdot \mathbf{n} & =g, & & \text { on } \Gamma, \\
u & =0, & & \text { on } \partial \Sigma .
\end{array}
$$

We define the operator $B_{0}$ in $\mathcal{L}\left(H^{-\frac{1}{2}}(\Gamma), \mathcal{V}^{\prime}\right)$ as $B_{0}=A_{0}^{b} N$. Its adjoint $B_{0}^{*}=N^{*} A_{0}^{b} \in \mathcal{L}\left(\mathcal{V}, H^{\frac{1}{2}}(\Gamma)\right)$ is exactly the trace application. We denote $\mathcal{U}=\mathrm{L}^{2}(\Gamma)$ equipped with the standard $\mathrm{L}^{2}$ scalar product. For all $g \in \mathcal{U}$ and all $u \in \mathcal{V}$, we have

$$
\left\langle B_{0} g, u\right\rangle_{\mathcal{V}^{\prime}, \mathcal{V}}=\left(g, B_{0}^{*} u\right)_{\mathcal{U}}
$$

Moreover, it is clear that $B_{0}$ also belongs to $\mathcal{L}\left(\mathcal{U}, \mathcal{V}^{\prime}\right)$ and its adjoint $B_{0}^{*}$ to $\mathcal{L}(\mathcal{V}, \mathcal{U})$. As mentioned in [35], one can see that

$$
Q_{0}=\left(A_{0}^{b}\right)^{-\frac{1}{2}} B_{0} \in \mathcal{L}(\mathcal{U}, \mathcal{H}), \quad Q_{0}^{*}=B_{0}^{*}\left(A_{0}^{b}\right)^{-\frac{1}{2}} \in \mathcal{L}(\mathcal{H}, \mathcal{U}), \quad Q_{0} Q_{0}^{*} \in \mathcal{L}(\mathcal{H}, \mathcal{H}) .
$$

We now introduce the state space $\mathcal{Y}^{b}=\mathcal{V} \times \mathcal{H} \times \mathcal{U}$ as the Hilbert space in which the solution $y=(u, v, \psi)$ is sought, and define the operator $A^{\mathrm{b}}: D\left(A^{\mathrm{b}}\right) \rightarrow \mathcal{Y}^{\mathrm{b}}$ as

$$
A^{b}=A^{\natural}-\beta B B^{*}=\left(\begin{array}{ccc}
0 & \mathbb{1} & 0 \\
-A_{0}^{b} & -\beta B_{0} B_{0}^{*} & -\sqrt{\delta} B_{0} \\
0 & \sqrt{\delta} B_{0}^{*} & 0
\end{array}\right)
$$

with

$$
A^{\natural}=\left(\begin{array}{ccc}
0 & \mathbb{1} & 0 \\
-A_{0}^{b} & 0 & -\sqrt{\delta} B_{0} \\
0 & \sqrt{\delta} B_{0}^{*} & 0
\end{array}\right), \quad B=\left(\begin{array}{c}
0 \\
B_{0} \\
0
\end{array}\right),
$$

and where

$$
D\left(A^{b}\right)=\left\{(u, v, \psi) \in \mathcal{Y}^{b}, v \in \mathcal{V}, A_{0}^{b} u+\beta B_{0} B_{0}^{*} v+\sqrt{\delta} B_{0} \psi \in \mathcal{H}\right\} .
$$

Then, upon the definition of adequate initial conditions, problem (75) corresponds to the first order problem (56) where the state is $y=\left(u, \partial_{t} u,\left.\sqrt{\delta} u\right|_{\Gamma}\right)$.

\subsubsection{The adjoint operator}

It is standard to show (see for instance [35]) that $A^{b}$ and $A^{b *}$ generate strongly continuous semi-groups of contraction. We give in the next paragraph the main idea to prove this result but first recall a definition of the adjoint operator $A^{\text {b* }}$. One can show the decomposition

$$
A^{b}=P_{0} A^{\sharp} P_{0} \quad \text { with } \quad P_{0}=\left(\begin{array}{ccc}
\mathbb{1} & 0 & 0 \\
0 & A_{0}^{b \frac{1}{2}} & 0 \\
0 & 0 & \mathbb{1}
\end{array}\right) \quad \text { and } \quad A^{\sharp}=\left(\begin{array}{ccc}
0 & \left(A_{0}^{b}\right)^{-\frac{1}{2}} & 0 \\
-A_{0}^{b^{\frac{1}{2}}} & -\beta Q_{0} Q_{0}^{*} & -\sqrt{\delta} Q_{0} \\
0 & \sqrt{\delta} Q_{0}^{*} & 0
\end{array}\right) \text {, }
$$

where $P_{0}$ and $A^{\sharp}$ satisfy

$$
P_{0} \in \mathcal{L}\left(D\left(A^{b}\right), D\left(A^{b}\right)\right) \cap \mathcal{L}\left(\mathcal{V} \times \mathcal{V} \times \mathcal{U}, \mathcal{Y}^{b}\right), \quad A^{\sharp} \in \mathcal{L}\left(D\left(A^{b}\right), \mathcal{V} \times \mathcal{V} \times \mathcal{U}\right)
$$

and

$$
D\left(A^{\sharp}\right)=\left\{y=(u, v, \psi) \in \mathcal{Y}^{b}, A_{0}^{b \frac{1}{2}} u+\beta Q_{0} Q_{0}^{*} v+\sqrt{\delta} Q_{0} \psi \in \mathcal{V}\right\} .
$$


Note that $P_{0}$ inherits the self-adjointness property of $A_{0}$. More precisely, for all $y$ and $q$ in $\mathcal{V} \times \mathcal{V} \times \mathcal{U}$ we have $\left(P_{0} y, q\right)_{\mathcal{Y}^{b}}=\left(y, P_{0} q\right)_{\mathcal{Y}^{b}}$. Remark also that the $A^{\sharp}$ can be extended to an operator in $\mathcal{L}\left(\mathcal{Y}^{b}, \mathcal{Y}^{b}\right)$ and its adjoint can be easily computed. We have indeed

$$
A^{\sharp *}=\left(\begin{array}{ccc}
0 & -\left(A_{0}^{b}\right)^{-\frac{1}{2}} & 0 \\
A_{0}^{b \frac{1}{2}} & -\beta Q_{0} Q_{0}^{*} & \sqrt{\delta} Q_{0} \\
0 & -\sqrt{\delta} Q_{0}^{*} & 0
\end{array}\right),
$$

with

$$
D\left(A^{\sharp *}\right)=\left\{y=(u, v, \psi) \in \mathcal{Y}^{b}, \quad A_{0}^{b \frac{1}{2}} u-\beta Q_{0} Q_{0}^{*} v+\sqrt{\delta} Q_{0} \psi \in \mathcal{V}\right\} .
$$

Now, we define by composition $A^{\text {b* }} \in \mathcal{L}\left(D\left(A^{\mathrm{b} *}\right), \mathcal{Y}^{\mathrm{b}}\right)$, namely

$$
A^{b *}=P_{0} A^{\sharp *} P_{0},
$$

where $P_{0}$ is now also seen as a bounded linear operator from $D\left(A^{b *}\right)$ to $D\left(A^{\sharp *}\right)$ with the definition

$$
D\left(A^{b *}\right)=\left\{y=(u, v, \psi) \in \mathcal{Y}^{b}, v \in \mathcal{V}, A_{0}^{b} u-\beta B_{0} B_{0}^{*} v-\sqrt{\delta} B_{0}^{*} \psi \in \mathcal{H}\right\} .
$$

Remark that there is only a sign change between the definition of $D\left(A^{\sharp}\right)$ and $D\left(A^{\sharp *}\right)$, and in between the definition of $D\left(A^{b}\right)$ and $D\left(A^{b *}\right)$, respectively. However this is sufficient for making the domain incompatible. Finally, one can check that for all $(y, q) \in D\left(A^{b}\right) \times D\left(A^{b *}\right)$, we have

$$
\left(A^{b} y, q\right)_{\mathcal{Y}}^{b}=\left(A^{\sharp} P_{0} y, P_{0} q\right)_{\mathcal{Y}}^{b}=\left(P_{0} y, A^{\sharp *} P_{0} q\right)_{\mathcal{Y}}^{b}=\left(y, A^{b *} q\right)_{\mathcal{Y}}^{b} .
$$

\subsubsection{Maximal dissipativity}

The proof of the lemma below being rather standard it is given in Appendix B for the sake of completeness.

Lemma 5.1. The operator $A^{b}: D\left(A^{b}\right) \rightarrow \mathcal{Y}^{b}$ is maximal dissipative.

By the Lumer and Phillips theorem, $A^{\text {b* }}$ is also maximal dissipative and generates a strongly continuous semi-group of contraction (see [7, Chapter 1, Theorem 2.8]). Therefore finally, Problem (75) enters the framework defined in Section 4.2 and the iterative back-and-forth algorithm can be applied.

\subsubsection{Initial data, strict and mild solutions}

We define the space $\mathcal{Y}_{0}^{b}$ of compactly supported initial data

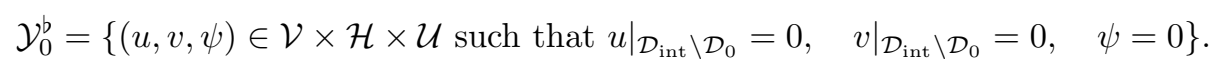

Thus, any solution $y=(u, v, \psi)$ of $(55)$ with $y_{0} \in \mathcal{Y}_{0}^{b}$ gives a solution $u$ of the wave propagation problem (75). More precisely, if $y$ is a strict solution we have

$$
u \in \mathrm{C}^{2}([0, T] ; \mathcal{H}) \cap \mathrm{C}^{1}([0, T] ; \mathcal{V}),
$$

whereas if $y$ is a mild solution then

$$
u \in \mathrm{C}^{1}([0, T] ; \mathcal{H}) \cap \mathrm{C}^{0}([0, T] ; \mathcal{V}) .
$$

\subsection{Application of the abstract back-and-forth algorithm}

\subsubsection{Admissibility}

It is useful in the next paragraph to give a meaning to the term $B^{*} y(t)$ when $y$ is a mild solution of problem (55). If $y(t)=(u(t), v(t), \psi(t))$ is a strict solution then we can consider the scalar product of $(56)$ with $y$ and get

$$
\frac{1}{2} \frac{d}{d t}\|y\|_{\mathcal{Y}^{\mathrm{b}}}=-\beta\left\|B_{0}^{*} v\right\|_{\mathcal{U}}^{2}
$$


because of the dissipative property of $A^{\text {b }}$ given by Lemma 5.1. Therefore

$$
\|y(t)\|_{\mathcal{Y}^{\mathfrak{b}}}=\left\|y_{0}\right\|_{\mathcal{Y}^{\mathrm{b}}}+2 \beta \int_{0}^{t}\left\|B_{0}^{*} v(s)\right\|_{\mathcal{U}}^{2} d s .
$$

The identity (77) shows that, for any strict solution $y(t)=e^{A^{b} t} y_{0}$ of (55) with $y_{0} \in D\left(A^{b}\right)$ we have,

$$
\forall t>0, \quad \beta \int_{0}^{t}\left\|B^{*} e^{A^{b} s} y_{0}\right\|_{\mathcal{U}}^{2} \leq \frac{1}{2}\left\|y_{0}\right\|_{\mathcal{Y}^{b}}
$$

Therefore, $B^{*}$ is admissible with respect to the semi-group $e^{A^{b} t}$ [52]. A direct consequence of the admissibility property (see [52, Proposition 4.3.2]) is that, for any given final time $T$ of observation, the application $B^{*} e^{A^{b} t} \in \mathcal{L}\left(D\left(A^{b}\right), \mathrm{L}^{2}(0, T) ; \mathcal{U}\right)$ has a unique continuous extension in $\mathcal{L}\left(\mathcal{Y}^{b}, \mathrm{~L}^{2}(0, T) ; \mathcal{U}\right)$. In other words, $(78)$ holds for $y_{0} \in \mathcal{Y}^{b}$ and corresponding $y(s)=e^{A^{b} s} y_{0}$ a mild solution of problem (55).

\subsubsection{Observation operator}

Our observations at hand correspond to the velocity $v=\partial_{t} u$ of a wave equation, set in an unbounded domain. To enter the theoretical framework presented above, we simply need to extend the definition of the observation operator $C$ to the augmented state $y=(u, v, \psi)$. Since $\psi(t)$ corresponds to $\left.\sqrt{\delta} u\right|_{\Gamma}(t)$ it is not observed, hence we define

$$
C^{b}=\left[\begin{array}{lll}
0 & \mathbb{1}_{\mid \mathcal{D}_{\text {obs }}} & 0
\end{array}\right]
$$

\subsubsection{Observability}

Our objective is now to prove the observability assumption (69) in order to justify that the reconstruction algorithm converges exponentially fast (in the absence of noise). We begin by showing that the application $B^{*} e^{A^{b} t}$ is bounded, in an adequate norm, by the measurements. More precisely, we have the following proposition.

Proposition 5.2. There exists $T>0$ and $\kappa>0$ such that, for all $y_{0} \in \mathcal{Y}_{0}^{b}$, we have

$$
\int_{0}^{T}\left\|C^{b} e^{A^{b} t} y_{0}\right\|_{\mathcal{Z}}^{2} \mathrm{~d} t \geq \kappa \int_{0}^{T}\left\|B^{*} e^{A^{b} t} y_{0}\right\|_{\mathcal{U}}^{2} \mathrm{~d} t
$$

Proof. We first consider a strict solution $y=(u, v, \psi)$ of equation (55) constructed by choosing $y_{0} \in D\left(A^{b}\right)$ (and $f=0)$. Then, by taking the scalar product of equation (55) with $y_{c}=\left(\chi_{c} u, \chi_{c} v, \psi\right)$ where $\chi_{c}$ is the smooth positive truncation function defined as in Section 3.3, it comes

$$
\begin{aligned}
\left(\dot{y}, y_{c}\right)_{\mathcal{Y}^{b}} & =\left(\nabla \dot{u}, \nabla\left(\chi_{c} u\right)\right)_{\mathrm{L}^{2}\left(\mathcal{D}_{\mathrm{int}}\right)}+\left(\dot{v}, \chi_{c} v\right)_{\mathrm{L}^{2}\left(\mathcal{D}_{\mathrm{int}}\right)}+\frac{1}{2} \frac{d}{d t}\|\psi\|_{\mathcal{U}}^{2} \\
& =\left(\nabla v, \nabla\left(\chi_{c} u\right)\right)_{\mathrm{L}^{2}\left(\mathcal{D}_{\mathrm{int}}\right)}+\left(-A_{0} u-\beta B_{0} B_{0}^{*} v-\sqrt{\delta} B_{0} \psi, \chi_{c} v\right)_{\mathcal{H}}+\sqrt{\delta}\left(B_{0}^{*} v, \psi\right)_{\mathcal{U}}
\end{aligned}
$$

Now, we observe that $v$ belongs to $\mathcal{V}$ and so does $\chi_{c} v$. Moreover, $B^{*}$ is the trace application, hence $B^{*} \chi_{c} v=B^{*} v$. Combining these observations, we have

$$
\begin{aligned}
\left(-A_{0} u-\beta B_{0} B_{0}^{*} v-\sqrt{\delta} B_{0} \psi, \chi_{c} v\right)_{\mathcal{H}} & =\left\langle-A_{0} u-\beta B_{0} B_{0}^{*} v-\sqrt{\delta} B_{0} \psi, \chi_{c} v\right\rangle_{\mathcal{V}^{\prime}, \mathcal{V}} \\
& =-\left(\nabla u, \nabla\left(\chi_{c} v\right)\right)_{\mathrm{L}^{2}\left(\mathcal{D}_{\mathrm{int}}\right)}-\beta\left\|B_{0}^{*} v\right\|_{\mathcal{U}}^{2}-\sqrt{\delta}\left(B_{0}^{*} v, \psi\right)_{\mathcal{U}} .
\end{aligned}
$$

Then, we know by the first equation of (55) that $\partial_{t} u=v$ in the domain and $\sqrt{\delta} u=\psi$ on the exterior boundary of the domain. Therefore, combining the two previous identities, we have that

$$
\frac{1}{2} \frac{\mathrm{d}}{\mathrm{d} t}\left(v, \chi_{c} v\right)_{\mathrm{L}^{2}\left(\mathcal{D}_{\text {int }}\right)}+\frac{1}{2} \frac{\mathrm{d}}{\mathrm{d} t}\left(\nabla u, \chi_{c} \nabla u\right)_{\mathrm{L}^{2}\left(\mathcal{D}_{\text {int }}\right)}+\frac{\delta}{2} \frac{\mathrm{d}}{\mathrm{d} t}\left\|\left.u\right|_{\Gamma}\right\|_{\mathcal{U}}^{2}+\beta\left\|B_{0}^{*} v\right\|_{\mathcal{U}}^{2}=-\left(\nabla u, v \nabla \chi_{c}\right)_{\mathrm{L}^{2}\left(\mathcal{D}_{\text {int }}\right)} .
$$


Integrating in time the above equation and recalling that $y(0)=y_{0}$ has its support in $\mathcal{D}_{0}$ and $\chi_{c}=0$ in $\mathcal{D}_{0}$, we obtain

$$
\begin{aligned}
\left(v(t), \chi_{c} v(t)\right)_{\mathrm{L}^{2}\left(\mathcal{D}_{\mathrm{int}}\right)}+\left(\nabla u(t), \chi_{c} \nabla u(t)\right)_{\mathrm{L}^{2}\left(\mathcal{D}_{\mathrm{int}}\right)} & +\delta\left\|\left.u(t)\right|_{\Gamma}\right\|_{\mathcal{U}}^{2} \\
& +2 \int_{0}^{t}\left\|B_{0}^{*} v(s)\right\|_{\mathcal{U}}^{2} \mathrm{~d} s=-2 \int_{0}^{t}\left(\nabla u(s), v(s) \nabla \chi_{c}\right)_{\mathrm{L}^{2}\left(\mathcal{D}_{\mathrm{int}}\right)} \mathrm{d} s .
\end{aligned}
$$

Then, using Cauchy-Schwarz and Young's inequalities, we have

$$
\left|\left(\nabla u, v \nabla \chi_{c}\right)_{\mathrm{L}^{2}\left(\mathcal{D}_{\mathrm{int}}\right)}\right| \leq \frac{\left\|\nabla \chi_{c}\right\|_{\infty}}{2}\left(\|\nabla u\|_{\mathrm{L}^{2}\left(\mathcal{D}_{c}\right)}^{2}+\|v\|_{\mathrm{L}^{2}\left(\mathcal{D}_{c}\right)}^{2}\right)
$$

Therefore, we deduce that

$$
2 \int_{0}^{t}\left\|B_{0}^{*} v(s)\right\|_{\mathcal{U}}^{2} \mathrm{~d} s \leq\left\|\nabla \chi_{c}\right\|_{\infty} \int_{0}^{t}\left(\|\nabla u(s)\|_{\mathrm{L}^{2}\left(\mathcal{D}_{c}\right)}^{2}+\|v(s)\|_{\mathrm{L}^{2}\left(\mathcal{D}_{c}\right)}^{2}\right) \mathrm{d} s .
$$

The rest of the proof relies on the energy equirepartition result of Appendix A. Finally, by a density argument we recover the announced result for all $y_{0}$ in $\mathcal{Y}_{0}^{b}$.

We can finally state the main observability result.

Proposition 5.3. There exists $T>0$ and $\tilde{\kappa}>0$ such that for all $y_{0} \in \mathcal{Y}_{0}^{b}$

$$
\int_{0}^{T}\left\|C^{b} e^{A^{\mathrm{b}} t} y_{0}\right\|_{\mathcal{Z}}^{2} \mathrm{~d} t+\int_{0}^{T}\left\|B^{*} e^{A^{\mathrm{b}} t} y_{0}\right\|_{\mathcal{U}}^{2} \mathrm{~d} t \geq \tilde{\kappa}\left\|y_{0}\right\|_{\mathcal{Y}^{\mathrm{b}}}^{2}
$$

We are going to give two different versions of the proof. A first proof takes advantage when $\delta=0$ of existing stabilization results under GCC in the case of a wave system in bounded domain with Neumann boundary conditions. The second proof in the more general case $\delta \neq 0$ directly justifies (80) using multipliers. Note that in this second case, microlocalization arguments could also be developed.

Proof. $\square$ By geometric control condition (GCC) in the case $\delta=0$. In the case $\delta=0$, we need to access a stability property for the error system

$$
\begin{array}{rr}
\partial_{t t} \tilde{u}-\Delta \tilde{u}+\mathbb{1}_{\mathcal{D}_{\mathrm{obs}}} \partial_{t} \tilde{u}=0, & \text { in }(0, T) \times \Omega \\
\tilde{u}=0, & \text { in }(0, T) \times \Sigma \\
\partial_{n} \tilde{u}=-\beta \partial_{t} \tilde{u}, & \text { in }(0, T) \times \Gamma
\end{array}
$$

We further assume that boundaries are smooth, which is typically the case in Figure 2.a. Therefore from $[5,36], \tilde{u}$ is exponentially stabilizable since the GCC is already satisfied for $\beta=0$ and $\partial_{n} u=-\beta \partial_{t} \tilde{u}$ gives additional stabilization alongs the rays [37]. In our abstract framework, this exactly means that $A_{\gamma}^{b}$ generates an exponentially stable semi-group and there exists $T>0$ and $\alpha>0$ such that for all $y_{0} \in \mathcal{Y}^{b}$,

$$
\int_{0}^{T}\left\|C^{b} e^{A_{\gamma}^{\mathrm{b}} t} y_{0}\right\|_{\mathcal{Z}}^{2} \mathrm{~d} t+\int_{0}^{T}\left\|B^{*} e^{A_{\gamma}^{\mathrm{b}} t} y_{0}\right\|_{\mathcal{U}}^{2} \mathrm{~d} t \geq \alpha\left\|y_{0}\right\|_{\mathcal{Y}^{\mathrm{b}}}^{2}
$$

The difficulty is now to obtain a similar inequality with $\gamma=0$. Following the proof of Lemma 4.4, we assume that $y_{0}$ belongs to $D\left(A^{b}\right) \cap \mathcal{Y}_{0}^{b}$ and we introduce the function $\tilde{y}$ defined by

$$
\tilde{y}=\left(e^{A^{b} t}-e^{A_{\gamma}^{b} t}\right) y_{0} \quad \in \quad \mathrm{C}^{1}\left([0, T] ; \mathcal{Y}^{b} \cap \mathrm{C}^{0}\left([0, T] ; D\left(A^{b}\right)\right),\right.
$$

then

$$
\dot{\tilde{y}}=A_{\gamma}^{b} \tilde{y}+\gamma C^{b, *} C^{b} e^{A^{b} t} y_{0} .
$$


Then using that $\tilde{y}(0)=0$ one can show, by standard energy estimates, the following inequality

$$
\|\tilde{y}(T)\|^{2}+\int_{0}^{T}\left\|B^{*} \tilde{y}(s)\right\|_{\mathcal{U}}^{2}+\left\|C^{b} \tilde{y}(s)\right\|_{\mathcal{Z}}^{2} \mathrm{~d} s \leq \int_{0}^{T}\left\|C^{b} e^{A^{b} s} y_{0}\right\|_{\mathcal{Z}}\left\|C^{b} \tilde{y}(s)\right\|_{\mathcal{Z}} \mathrm{d} s .
$$

Using Young's inequality $2 a b \leq \frac{a^{2}}{\nu^{2}}+b^{2} \nu^{2}$ ) with $0<\nu<1$, we get

$$
\|\tilde{y}(T)\|^{2}+\int_{0}^{T}\left\|B^{*} \tilde{y}(t)\right\|_{\mathcal{U}}^{2}+\left(1-\nu^{2}\right)\left\|C^{b} \tilde{y}(t)\right\|_{\mathcal{Z}}^{2} \mathrm{~d} t \leq \frac{1}{\nu^{2}} \int_{0}^{T}\left\|C^{b} e^{A^{b} t} y_{0}\right\|_{\mathcal{Z}}^{2} \mathrm{~d} t .
$$

Therefore, there exists $\tilde{\alpha}$ such that

$$
\int_{0}^{T}\left\|B^{*} \tilde{y}(t)\right\|_{\mathcal{U}}^{2}+\left\|C^{b} \tilde{y}(t)\right\|_{\mathcal{Z}}^{2} \mathrm{~d} t \leq \tilde{\alpha} \int_{0}^{T}\left\|C^{b} e^{A^{b} t} y_{0}\right\|_{\mathcal{Z}}^{2} \mathrm{~d} t .
$$

We thus deduce from (81) and $\|a+b\|^{2} \leq 2\|a\|^{2}+2\|b\|^{2}$ that

$$
\begin{aligned}
\alpha\left\|y_{0}\right\|_{\mathcal{Y}^{b}} & \leq \int_{0}^{T}\left\|C^{b} e^{A_{\gamma}^{b} t} y_{0}\right\|_{\mathcal{Z}}^{2}+\left\|B^{*} e^{A_{\gamma}^{b} t} y_{0}\right\|_{\mathcal{U}}^{2} \mathrm{~d} t \\
& \leq 2 \int_{0}^{T}\left\|C^{b} e^{A^{b} t} y_{0}\right\|_{\mathcal{Z}}^{2}+\left\|B^{*} e^{A^{b} t} y_{0}\right\|_{\mathcal{U}}^{2} \mathrm{~d} t+2 \int_{0}^{T}\left\|C^{b} \tilde{y}(t)\right\|_{\mathcal{Z}}^{2}+\left\|B^{*} \tilde{y}(t)\right\|_{\mathcal{U}}^{2} \mathrm{~d} t \\
& \leq 2 \int_{0}^{T}(1+\tilde{\alpha})\left\|C^{b} e^{A^{b} t} y_{0}\right\|_{\mathcal{Z}}^{2}+\left\|B^{*} e^{A^{b} t} y_{0}\right\|_{\mathcal{U}}^{2} \mathrm{~d} t .
\end{aligned}
$$

The proof is concluded by density of $D\left(A^{b}\right) \cap \mathcal{Y}_{0}^{b}$ into $\mathcal{Y}_{0}^{b}$.

By multipliers in the general case $\delta \neq 0$. We assume that $y_{0}$ belongs to $D\left(A^{b}\right) \cap \mathcal{Y}_{0}^{b}$ then $y=e^{A^{b} t} y_{0}$ is a strict solution of the problem $\dot{y}=A^{b} y$ and therefore $y=(u, v, \psi)$ with

$$
u \in \mathrm{C}^{2}\left([0, T] ; \mathrm{L}^{2}\left(\mathcal{D}_{\text {int }}\right)\right) \cap \mathrm{C}^{1}\left([0, T] ; \mathrm{H}^{1}\left(\mathcal{D}_{\text {int }}\right)\right), \quad v=\partial_{t} u, \quad \psi=\left.\sqrt{\delta} u\right|_{\Gamma}
$$

satisfies the homogeneous wave equation in $\mathcal{D}_{\text {int }}$. Therefore, we can apply a classical multiplier result that we recall in Appendix C Theorem C.2 for the sake of completeness: There exists $\kappa$ and $\tilde{\kappa}$ such that for all $T>0$ we have

$$
\frac{T}{2} \mathcal{E}(0)-\kappa(\mathcal{E}(T)+\mathcal{E}(0)) \leq \tilde{\kappa} \int_{\mathcal{D}_{c} \times(0, T)}\left|\partial_{t} u\right|^{2}+|\nabla u|^{2} \mathrm{~d} \mathbf{x} \mathrm{d} t
$$

with

$$
\mathcal{E}(t)=\left\|\partial_{t} u(t)\right\|_{\mathrm{L}^{2}(\Omega)}^{2}+\|\nabla u(t)\|_{\mathrm{L}^{2}(\Omega)}^{2} .
$$

Note that it is important here that $\kappa$ does not depend on $T$. Thanks to standard energy estimates of the wave equation problem we can deduce that

$$
\mathcal{E}(T) \leq \mathcal{E}(T)+\delta\left\|\left.u(T)\right|_{\Gamma}\right\|_{L^{2}(\Gamma)}^{2} \leq \mathcal{E}(0)+\delta\left\|\left.u(0)\right|_{\Gamma}\right\|_{L^{2}(\Gamma)}^{2}=\mathcal{E}(0) .
$$

Then, for $T$ sufficiently large, one can show that the left-hand side of (82) can be bounded from below, by $\hat{\kappa} \mathcal{E}(0)$ where $\hat{\kappa}$ depends on $T, \kappa$ and $\delta$. The proof is concluded by proving a result of energy equirepartition following the lines of the proof of Lemma 3.8 (again we omit this part for the sake of conciseness). Finally, the density of $D\left(A^{b}\right) \cap \mathcal{Y}_{0}^{b}$ into $\mathcal{Y}_{0}^{b}$ enables us to show that the observability inequality holds for $y_{0} \in \mathcal{Y}_{0}^{b}$.

We can now conclude this section by the expected observability result. 
Corollary 5.4. There exist $T>0$ and $\kappa$ such that for all $y_{0} \in \mathcal{Y}_{0}^{b}$ we have

$$
\int_{0}^{T}\left\|C^{b} e^{A^{b} t} y_{0}\right\|_{\mathcal{Z}}^{2} \mathrm{~d} t \geq \kappa\left\|y_{0}\right\|_{\mathcal{Y}^{b}}^{2}
$$

Proof. Here, we combine the results of Propositions 5.2 and 5.3. There exist $T>0, \alpha>0$ and $\tilde{\alpha}>0$ such that

$$
\begin{aligned}
\int_{0}^{T}\left\|C^{b} e^{A^{b} t} y_{0}\right\|_{\mathcal{Z}}^{2} \mathrm{~d} t & \geq \frac{1}{2} \int_{0}^{T}\left\|C^{b} e^{A^{b} t} y_{0}\right\|_{\mathcal{Z}}^{2} \mathrm{~d} t+\frac{1}{2 \alpha} \int_{0}^{T}\left\|B^{*} e^{A^{b} t} y_{0}\right\|_{\mathcal{U}}^{2} \mathrm{~d} t \\
& \geq \min \left(\frac{1}{2}, \frac{1}{2 \alpha}\right) \tilde{\alpha}\left\|y_{0}\right\|_{\mathcal{Y}^{b}}^{2}
\end{aligned}
$$

which gives (83).

Remark 5.5. We point out that our approach is only based on the dissipative property of the associated boundary condition, namely the fact that they are absorbing. Therefore, our result could be applied to quite general approximated transparent boundary condition. In particular in $1 \mathrm{D}$, this could be applied to Perfect Match Layer (PML) [9] conditions as we have the same type of dissipative property [24]. However, the question of the dissipative property of the PML is still open in dimension greater than one.

\section{NumericAl inVEStigations}

The time and space discretizations of feedback control formulations is a classical issue and our problem faces the same difficulties as for bounded domain formulations, with additionally the question about the discretization of transparent boundary conditions. To briefly summarize the common issues, many discretizations strategies - including the conservative ones - lead to the introduction of spurious high frequencies that are not stabilized by the feedback and pollute the reconstruction, see for instance [4,53]. Classical remedies are based on the introduction of some small numerical viscosity $[14,23]$ or by considering well-tuned spatial discretization based on non-uniform meshes [21,39], or on mixed finite elements [15,25]. In this section, we will numerically realize that the same difficulties and remedies works in our unbounded domain configuration. However, proceeding to the complete numerical analysis of our back-and-forth observer as it is the case for bounded domains in [32] will be the subject of a future work. Note that most of the proofs in bounded domain configurations are based on spectral arguments [22,42], hence justifying our effort in the previous sections to first bound the domain with artificial boundary conditions. In other words, Section 4 has paved the way for future numerical analysis of the problem.

\subsection{The $1 \mathrm{D}$ case}

In this first paragraph, we consider the half-line $\mathcal{D}=\mathbb{R}^{+}$which will enable us to illustrate and emphasize some properties of the proposed algorithm. The bounded domain is taken as the segment $(0,1)$ with a transparent boundary condition at $x=1$ and homogeneous Dirichlet condition at $x=0$. As already mentioned in Section 4.1, the transparent condition in the 1D case is well-known, namely

$$
\partial_{x} u(1, t)=-\partial_{t} u(1, t), \quad t \in(0, T),
$$

and can be easily implemented. To discretize the forward observer (50) and the corresponding adjoint dynamics (53a)-(53b), we use classical Lagrange finite elements for the space variable and a mid-point scheme for the time variable [14]. More precisely we denote by $V_{h}$ the discretized space associated with $V=\left\{u \in \mathrm{H}^{1}\right.$ s.t. $\left.u(0)=0\right\}$. The number of degrees of freedom for $V_{h}$ is $N_{h}$, hence for all $u_{h} \in V_{h}$ we associate a corresponding vector $\mathrm{u}_{h} \in \mathbb{R}^{N_{h}}$ and we denote by $r_{h}$ the Lagrange interpollant from $V$ to $V_{h}$. Then the fully discretized system reads

$$
\mid \begin{aligned}
& I_{h}\left(\frac{\mathrm{y}_{h}^{k+1}-\mathrm{y}_{h}^{k}}{\Delta t}\right)=A_{h}^{\mathrm{b}}\left(\frac{\mathrm{y}_{h}^{k+1}+\mathrm{y}_{h}^{k}}{2}\right)+B_{h} B_{h}^{\top}\left(\frac{\mathrm{y}_{h}^{k+1}+\mathrm{y}_{h}^{k}}{2}\right) \\
& \mathrm{y}_{h}^{0}=\mathrm{r}_{h}\left(y_{0}\right)
\end{aligned}
$$


where

with

$$
I_{h}=\left(\begin{array}{cc}
\mathbb{K} & 0 \\
0 & \mathbb{M}
\end{array}\right), \quad A_{h}^{b}=\left(\begin{array}{cc}
0 & \mathbb{K} \\
-\mathbb{K} & 0
\end{array}\right), \quad B_{h}=\left(\begin{array}{c}
0 \\
\delta_{N_{h}}
\end{array}\right),
$$

$$
\forall\left(v_{h}, u_{h}\right) \in V_{h}^{2}, \quad \mathbf{v}_{h}^{\top} M^{M} u_{h}=\int_{0}^{1} v_{h}(x) u_{h}(x) \mathrm{d} x, \quad \mathbf{v}_{h}^{\top} \mathbb{K} u_{h}=\int_{0}^{1} v_{h}^{\prime}(x) u_{h}^{\prime}(x) \mathrm{d} x,
$$

and $\delta_{N_{h}}=(0 \cdots 01)^{\top}$. We take as time step $\Delta t=0.005$, final time $T=1$ and as spatial step $\Delta x=0.005$. We consider two kinds of meshes: a uniform mesh, i.e. $x_{i}=i \Delta x$, and a non-uniform mesh $y_{i}=g\left(x_{i}\right)$ as proposed in [39]. In the numerical examples, we choose $g(x)$

$$
g(x)=\left\{\begin{array}{lll}
\frac{1+\sqrt{2 x-1}}{2} & \text { if } & 2 x-1 \geq 0 \\
\frac{1-\sqrt{1-2 x}}{2} & \text { if } & 2 x-1<0
\end{array}\right.
$$

leading to the mesh pictured in Figure 4.b. The observation region is given by $\mathcal{D}_{\text {obs }}=(0,0.2) \cup(0.8,1)$ and

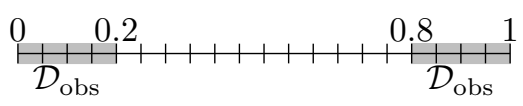

(a)

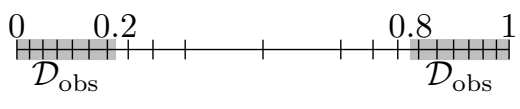

(b)

FIgURE 4. 1D mesh : (a) uniform mesh, (b) non-uniform mesh.

we assume the initial state to be in $\mathcal{D}_{0}=(0,0.95)$. Therefore, the discretized observation operator is given by

$$
C_{h}^{b}=\left(\begin{array}{ll}
0 & \delta_{\left(x_{i} \in \mathcal{D}_{\text {obs }}\right)}
\end{array}\right) .
$$

We define also the mass matrices in the observation space

$$
\forall\left(v_{h}, u_{h}\right) \in V_{h}^{2}, \quad \mathbf{v}_{h}^{\top} \mathbb{M}_{\mathrm{obs}} \mathrm{u}_{h}=\int_{(0,0.2) \cup(0.8,1)} v_{h}(x) u_{h}(x) \mathrm{d} x .
$$

Finally, we define the approximation $\Pi_{h}^{b}$ of the projection $\Pi^{b}$ operator by solving

$$
\Pi_{h}^{b}=\left(I_{h}^{0}\right)^{-1} I_{h}, \quad \text { with } I_{h}^{0}=\left(\begin{array}{cc}
\mathbb{K}_{0} & 0 \\
0 & \mathbf{M}_{0}
\end{array}\right),
$$

where $\mathbb{K}_{0}$ and $\mathbb{M}_{0}$ are the mass matrix and stiffness matrix built from shape functions with support in $\mathcal{D}_{0}$

The discretization of the reconstruction algorithm (68) then reads

$$
\begin{aligned}
& \mid I_{h}\left(\frac{\hat{\mathrm{y}}_{h}^{k+1,(n)}-\hat{\mathrm{y}}_{h}^{k,(n)}}{\Delta t}\right)=A_{h}^{b}\left(\frac{\hat{\mathrm{y}}_{h}^{k+1,(n)}+\hat{\mathrm{y}}_{h}^{k,(n)}}{2}\right)+B_{h} B_{h}^{\top}\left(\frac{\hat{\mathrm{y}}_{h}^{k+1,(n)}+\hat{\mathrm{y}}_{h}^{k,(n)}}{2}\right) \\
& +\gamma C_{h}^{\mathrm{b}} \mathrm{M}_{\mathrm{obs}}\left(\mathrm{r}_{h}\left(z\left(t_{k}+\frac{\Delta t}{2}\right)\right)-C_{h}^{\mathrm{b}}\left(\frac{\hat{\mathrm{y}}_{h}^{k+1,(n)}+\hat{\mathrm{y}}_{h}^{k,(n)}}{2}\right)\right), \\
& \hat{\mathrm{y}}^{0,(n)}=\Pi_{h}^{\mathrm{b}}\left(\hat{\mathrm{y}}^{0,(n-1)}-2 \mathrm{q}^{0,(n-1)}\right), \\
& I_{h}\left(\frac{\mathrm{q}_{h}^{k+1,(n)}-\mathrm{q}_{h}^{k,(n)}}{\Delta t}\right)+A_{h}^{\mathrm{b}}\left(\frac{\mathrm{q}_{h}^{k+1,(n)}+\mathrm{q}_{h}^{k,(n)}}{2}\right) \\
& =-\gamma C_{h}^{b} \boldsymbol{\top} M_{\mathrm{obs}}\left(\mathrm{r}_{h}\left(z\left(t_{k}+\frac{\Delta t}{2}\right)-C_{h}^{b}\left(\frac{\hat{\mathrm{y}}_{h}^{k+1,(n)}+\hat{\mathrm{y}}_{h}^{k,(n)}}{2}\right)\right),\right. \\
& \mathrm{q}^{N_{T},(n)}=0,
\end{aligned}
$$

$\left(\operatorname{Ad}_{h}^{b} \& \mathrm{~F}_{h}^{b}+\operatorname{Proj}\right)$ 
It is worth noticing that we choose to discretize the adjoint (59) instead of the backward dynamics (59). The backward observer is then defined implicitly as in (60) by

$$
\hat{\mathrm{y}}_{b, h}=\hat{\mathrm{y}}_{h}+2 \mathrm{q}_{h} .
$$

Indeed, using the adjoint instead of the backward observer presents some advantages. Firstly, the condition $q_{h}(T)=0$ is simpler than the final condition of the backward observer. Secondly, the adjoint dynamics does not require to store the discretized quantity $B_{h} B_{h}^{*} \hat{y}_{h}$, but only $r_{h}(z)-C_{h} \hat{y}_{h}$, with $r_{h}$ the discretization interpolant. This quantity has indeed the same memory storage volume as the already stored available measurements.

In order to show the discretized equivalent of the error reconstruction operator (64) (see Proposition (4.2)), we first assume that the data are perfectly produced by the discretized system (84) namely we take

$$
r_{h}\left(z\left(t_{k}+\frac{\Delta t}{2}\right)\right)=C_{h}^{b}\left(\frac{\mathrm{y}_{h}^{k+1,(n)}+\mathrm{y}_{h}^{k,(n)}}{2}\right) .
$$

Then, introducing the two matrices

$$
\Phi_{0, h}=\frac{I_{h}}{\Delta t}+\frac{A_{h}}{2}+\gamma \frac{C_{h}^{b} \mathrm{M}_{\mathrm{obs}} C_{h}^{b}}{2}+\frac{B_{h} B_{h}^{\top}}{2} \quad \text { and } \quad \Phi_{1, h}=\frac{I_{h}}{\Delta t}-\frac{A_{h}}{2}-\gamma \frac{C_{h}^{\mathrm{b} \boldsymbol{\top} \mathrm{M}_{\mathrm{obs}} C_{h}^{b}}}{2}-\frac{B_{h} B_{h}^{\top}}{2},
$$

we easily deduces after a single back-and-forth iteration that

$$
\mathrm{q}_{h}^{k,(1)}=\gamma \sum_{j=n}^{N-1}\left[\Phi_{0, h}^{-1} \Phi_{1, h}\right]^{j-n} \Phi_{0, h}^{-1} C_{h}^{b \top} \mathbb{M}_{\mathrm{obs}} C_{h}^{b}\left(\frac{\tilde{\mathrm{y}}_{h}^{j+1,(1)}+\tilde{\mathrm{y}}_{h}^{j,(1)}}{2}\right) .
$$

Recalling that we define at the discretized level the same quantity as in the continuous framework, namely

$$
\tilde{\mathrm{y}}_{h}^{k,(n)}=\hat{\mathrm{y}}_{h}^{k,(n)}-\mathrm{y}_{h}^{k,(n)} \quad \text { and } \quad \tilde{\mathrm{y}}_{b, h}^{k,(n)}=\tilde{\mathrm{y}}_{h}^{k,(n)}+2 \mathrm{q}_{h}^{k,(n)}\left(=\hat{\mathrm{y}}_{b, h}^{k,(n)}-\mathrm{y}_{h}^{k,(n)}\right),
$$

we have by simple manipulations of the discrete operator - in fact using discrete Duhamel formula,

$$
\begin{aligned}
\tilde{\mathrm{y}}_{b, h}^{0,(1)} & =\tilde{\mathrm{y}}_{h}^{0,(0)}-2 \gamma \sum_{j=0}^{N_{T}-1}\left[\Phi_{0, h}^{-1} \Phi_{1, h}\right]^{j} \Phi_{0, h}^{-1} C_{h}^{\mathrm{b}} \mathrm{M}_{\mathrm{obs}} C_{h}^{b}\left(\frac{\tilde{\mathrm{y}}_{h}^{j+1,(1)}+\tilde{\mathrm{y}}_{h}^{j,(1)}}{2}\right) \\
& =\tilde{\mathrm{y}}_{h}^{0,(0)}-2 \gamma \sum_{j=0}^{N_{T}-1}\left[\Phi_{0, h}^{-1} \Phi_{1, h}\right]^{j} \Phi_{0, h}^{-1} C_{h}^{\mathrm{\top}} \mathrm{M} \mathrm{M}_{\mathrm{obs}} C_{h}^{b}\left[\Phi_{0, h}^{-\boldsymbol{\top}} \Phi_{1, h}^{\top}\right]^{j}\left[\mathbb{1}+\Phi_{0, h}^{-\boldsymbol{\top}} \Phi_{1, h}^{\top}\right] \tilde{\mathrm{y}}_{h}^{0,(0)}
\end{aligned}
$$

which leads to the following proposition.

Proposition 6.1. The error of reconstruction is given at each time step by

$$
\tilde{\mathrm{y}}_{h}^{0,(k+1)}=\Pi_{h}^{\mathrm{b}} \Lambda_{\gamma, h, \Delta t} \Pi_{h}^{\mathrm{b}} \tilde{\mathrm{y}}_{h}^{0,(k)}
$$

where

$$
\Lambda_{\gamma, h, \Delta t}=\mathbb{1}-2 \gamma \sum_{j=0}^{N-1} \Phi_{0, h}^{-1}\left[\Phi_{1, h} \Phi_{0, h}^{-1}\right]^{j} C_{h}^{b} \boldsymbol{\top} \mathbb{M}_{o b s} C_{h}^{b}\left[\Phi_{0, h}^{-\boldsymbol{\top}} \Phi_{0, h}^{\top}\right]^{j} \Phi_{0, h}^{-\boldsymbol{\top}} I_{h},
$$

is a symmetric matrix with respect to the scalar product defined by $I_{h}$, positive and of norm smaller than one, and so is $\Pi_{h}^{b} \Lambda_{\gamma, h, \Delta t} \Pi_{h}^{b}$.

Proof. From the error (86), it comes

$$
\Lambda_{\gamma, h, \Delta t}=\mathbb{1}-2 \gamma \sum_{j=0}^{N_{T}-1}\left[\Phi_{0, h}^{-1} \Phi_{1, h}\right]^{j} \Phi_{0, h}^{-1} C_{h}^{b \boldsymbol{\top}} \mathbb{M}_{\mathrm{obs}} C_{h}^{b}\left[\Phi_{0, h}^{-\top} \Phi_{1, h}^{\top}\right]^{j}\left[\mathbb{1}+\Phi_{0, h}^{-\top} \Phi_{1, h}^{\top}\right] .
$$


We then simply need to notice that

$$
\left[\Phi_{0, h}^{-1} \Phi_{1, h}\right]^{j} \Phi_{0, h}^{-1}=\Phi_{0, h}^{-1}\left[\Phi_{1, h} \Phi_{0, h}^{-1}\right]^{j},
$$

and

$$
\left[\mathbb{1}+\Phi_{0, h}^{-\top} \Phi_{1, h}^{\top}\right]=\Phi_{0, h}^{-\boldsymbol{\top}}\left[\Phi_{0, h}^{\top}+\Phi_{1, h}^{\top}\right]=\Phi_{0, h}^{-\top} I_{h} .
$$

Therefore $I_{h} \Lambda_{\gamma, h, \Delta t}$ is symmetric positive and of norm smaller than one, and so is $\Pi_{h}^{b} \Lambda_{\gamma, h, \Delta t} \Pi_{h}^{b}$.

In the general case where $r_{h}\left(z\left(t_{n}+\frac{\Delta t}{2}\right)\right)$ comes from real measurements, we need to take into account the complete identity

$$
r_{h}\left(z\left(t_{n}+\frac{\Delta t}{2}\right)\right)=C_{h}^{b}\left(\frac{\mathrm{y}_{h}^{k+1,(n)}+\mathrm{y}_{h}^{k,(n)}}{2}\right)+\eta_{m}+\eta_{b}+\eta_{h}
$$

where $\eta_{m}$ is the observation noise, $\eta_{b}$ comes from the approximation error induced by the artificial boundary conditions, and $\eta_{h}$ from the discretization error. Therefore one can expect that (87) becomes

$$
\tilde{\mathrm{y}}_{h}^{0,(k+1)}=\Lambda_{\gamma, h, \Delta t} \tilde{\mathrm{y}}_{h}^{0,(k)}+\epsilon,
$$

where $\epsilon$ is consistency error that combines the observation noise, the approximation error induced by the artificial boundary conditions, and the discretization error. However, such a complete numerical analysis goes beyond the focus of the present article.

\subsubsection{Reconstruction performance with respect to gain variations}

First, let us illustrate some properties of $\Lambda_{\gamma}$. As we have seen, this operator has a norm smaller than one and is a contraction if the observability inequality is satisfied. As mentioned in the introduction of this section, the discretization process can destroy the contraction property even with conservative discretization choices. This is illustrated in Figure 5 where we plot the spectral radius (equivalently its norm) of the symmetric matrix corresponding to the discretized operator $\Pi_{h}^{b} \Lambda_{\gamma, h, \Delta t} \Pi_{h}^{b}$ with respect to the gain parameter $\gamma$. We note that, in the first situation with a uniform mesh, the norm of the matrix is always equal to 1 . This is due to spurious high frequency modes which are not stabilized. On the other hand, in the second case using a non-uniform mesh as proposed in [39] for 1D bounded domain, the norm is strictly smaller than one (for $\gamma>0$ ). Moreover, there is a best value of $\gamma \sim 14$ for which the spectral radius is the smallest. Then, for larger $\gamma$ the norm increases which is the well-known phenomenon of "over-damping" phenomena in stabilization [47] and its consequences for observation [14].

\subsubsection{Algorithm convergence using perfect data}

We consider the situation where we want to reconstruct a gaussian as an initial state, namely

$$
y_{0}=\left(u_{0}(x), 0\right) \quad \text { with } \quad u_{0}(x)= \begin{cases}e^{-(30(x-0.2))^{2}} & \text { if } \quad x \in \mathcal{D}_{0} \\ 0 & \text { if not }\end{cases}
$$

given the exact data $z$ (the data are generated with the same discretization parameters as the observers). We initialize the Back \& Forth Algorithm with null initial state and choose a rather optimal $\gamma=14$.

In Figure 6, we can see that the algorithm convergence is fully achieved only when taking a non-uniform mesh and applying the projection operator. This emphasizes the importance of the projection step. Moreover, we can verify that the convergence slope - i.e. 0.45 - corresponds in this case to the $(\log 10)$ of the norm of the matrix operator $\Pi_{h}^{b} \Lambda_{\gamma, h, \Delta t} \Pi_{h}^{b}$ obtained in Figure 5 for $\gamma=14$.

Once again, to illustrate the importance of the projection step and in order to have a better understanding of the convergence results, we have represented in Figure 7 the velocity obtained at the end of the first 10 iterations of the algorithm. As we can see, without projection, the error focus on the boundary $x=1$ of the domain where it cannot be damped. This illustrates the necessity to have $\mathcal{D}_{0}$ strictly included in the 


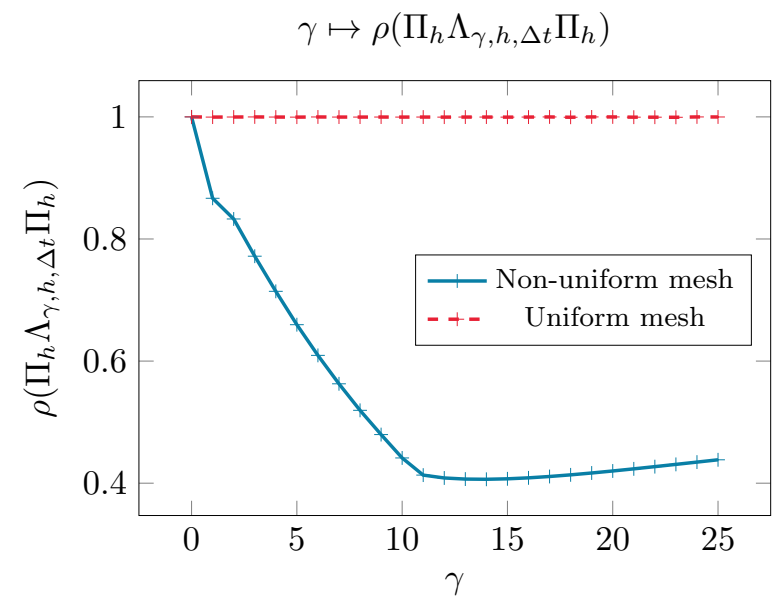

FiguRE 5. Norm of the matrix $\Pi_{h}^{b} \Lambda_{\gamma, h, \Delta t} \Pi_{h}^{b}$ versus the gain parameter $\gamma$.
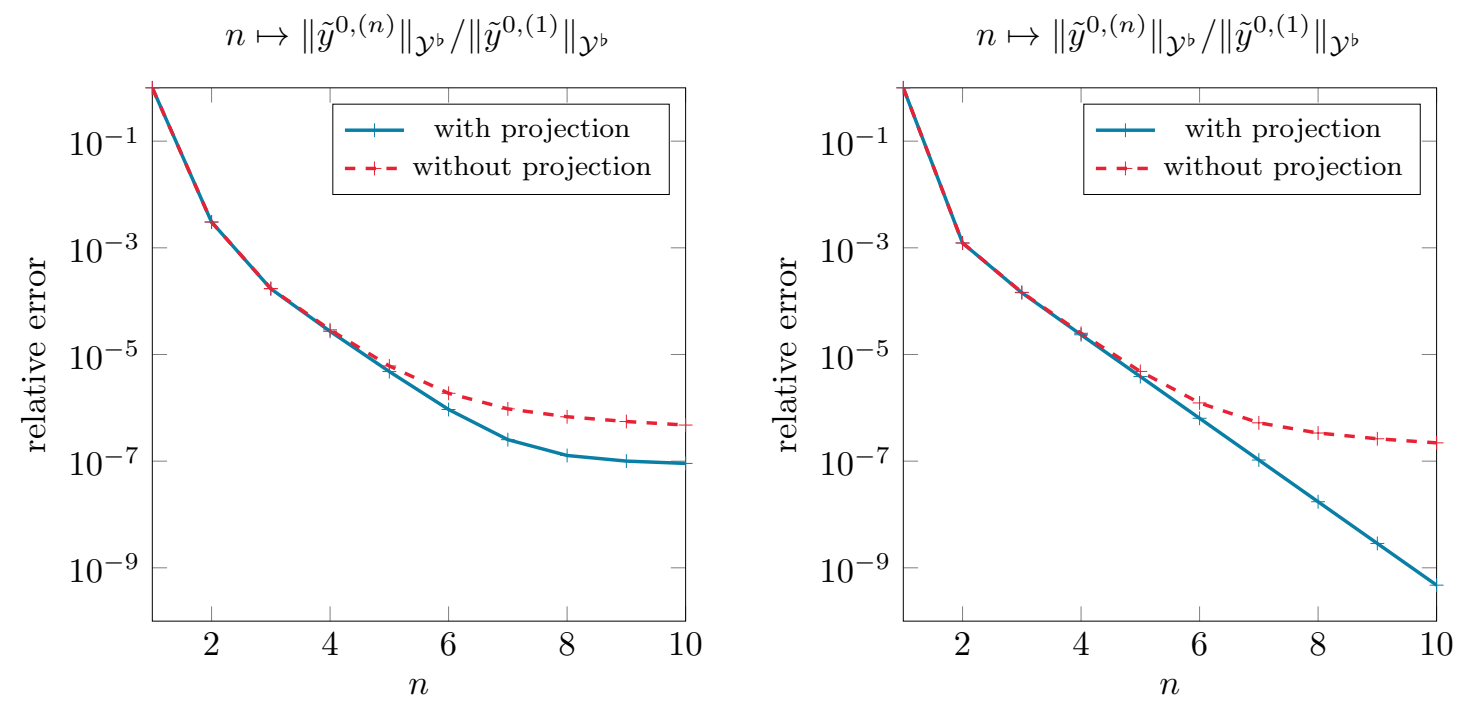

FiguRE $6 . \log _{10}$ of the error versus the number of iterations in the case of perfect observations data: On the left with the uniform mesh, On the right, with the non-uniform mesh.

domain surrounded by $\mathcal{D}_{\text {obs. }}$. We also notice the spurious highly oscillating modes in the case of uniform mesh survive to the projection step, hence concentrate in the middle of the domain.

\subsubsection{Reconstruction algorithm using noisy data}

Let us now illustrate a convergence result in the case of noisy data. The observations have been generated using $\Delta x=\Delta t=0.005$, with a non-uniform mesh using the function $g_{2}(x)=\frac{1}{2}(\sin ((2 x-1) \pi)+1)$, and have been perturbed by a high frequency perturbation of jump type on each node and amplitude $5 \%$ of the maximal amplitude of the initial condition. For the observers, we used $\Delta x=0.001$ and $\Delta t=0.005$.

As we can see in Figure 9, the algorithm converges very fast. Moreover as predicted, using a non-uniform mesh optimizes the convergence. In the case of a uniform mesh, after the first iteration, the error grows (very) slowly. 

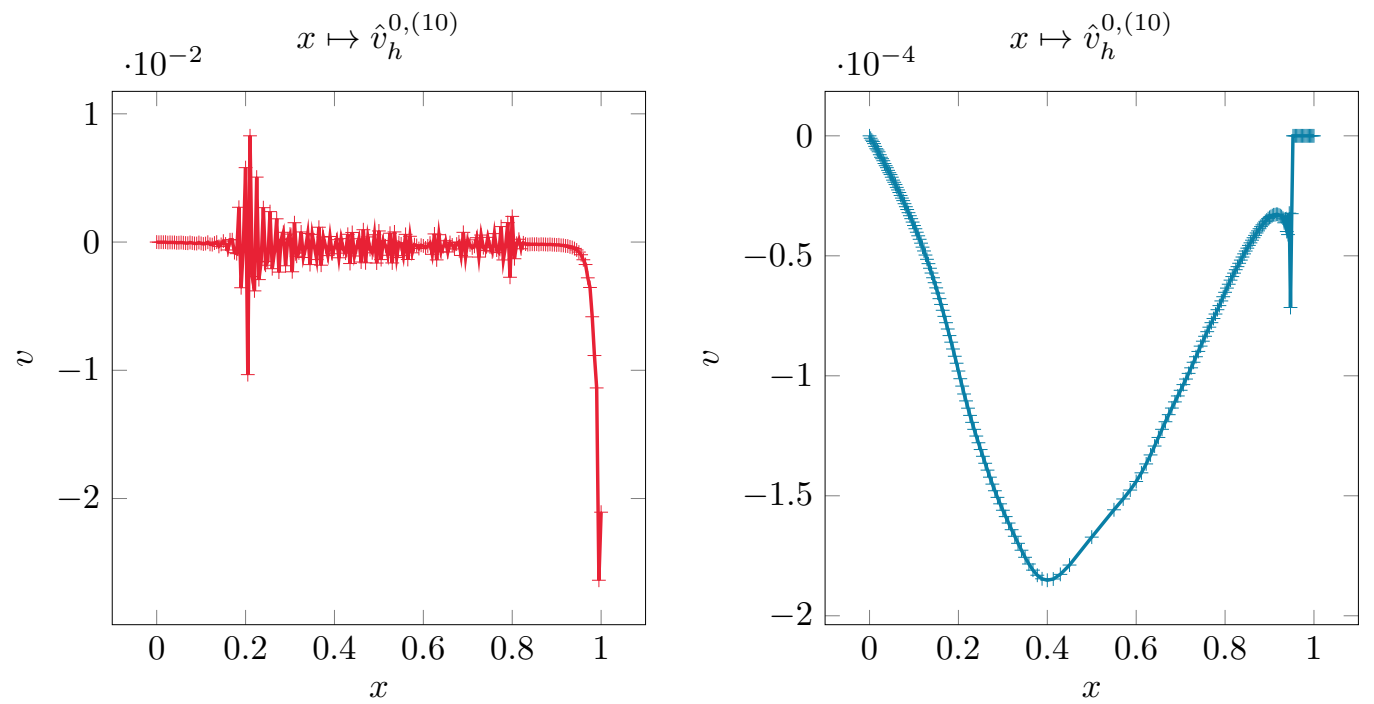

FiguRE 7. Initial velocity reconstruction after 10 iterations: (left) uniform mesh without projection; (right) non-uniform mesh with projection.
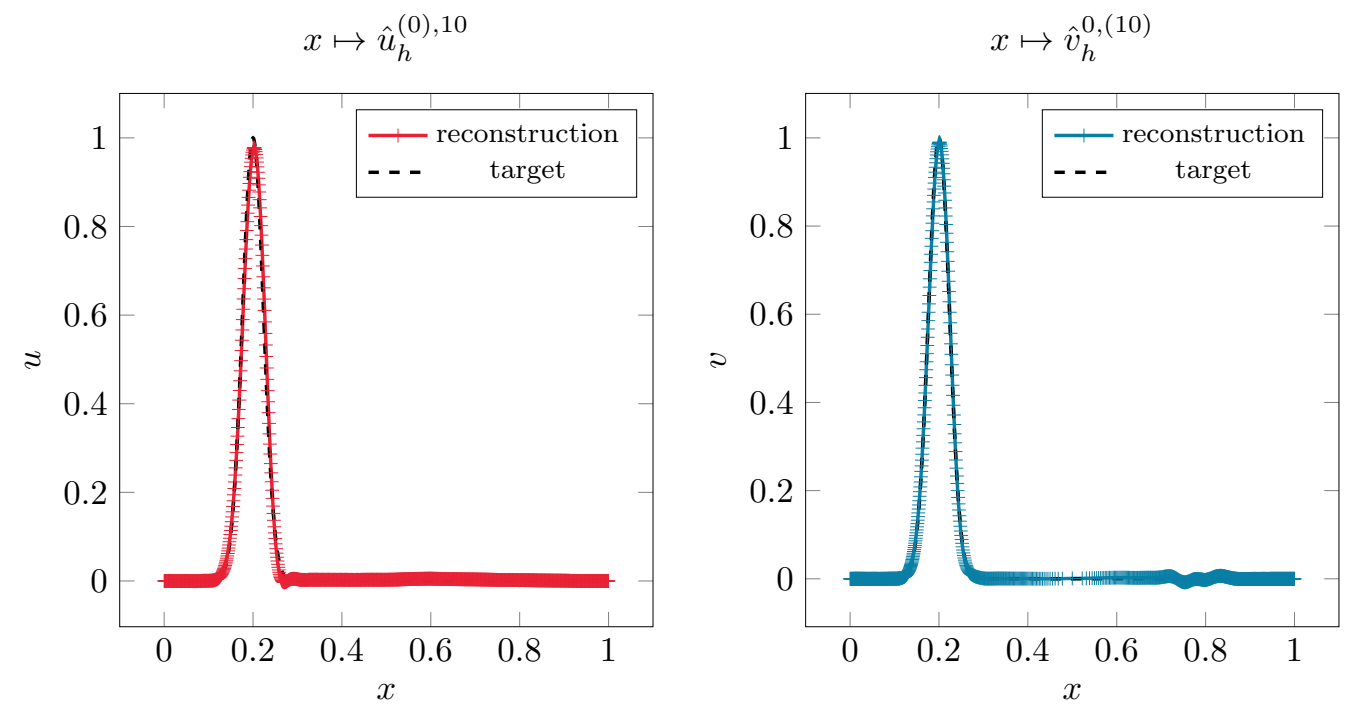

FiguRE 8. Reconstruction of the initial field after 10 iterations in the case of noisy data observations: (left) uniform mesh ; (right) non-uniform mesh

\subsubsection{Reconstruction using approximate boundary condition}

Althought in the 1D case, as we mentionned, the transparent boundary condition is known and can be implemented, let us show some results in the case of approximate boundary conditions. We consider the following boundary condition:

$$
\partial_{n} u(1, t)=-\beta \partial_{t} u(1, t), \quad t \in(0, T),
$$

where $\beta \in[0,1]$ is a parameter $(\beta=0$ corresponding to homogeneous Neumann boundary condition and $\beta=1$ to the exact transparent boundary condition). In Figure 10, we can observe that the best norm of the reconstruction operator can be achieved taking $\beta=0$ (note that all results are obtained with non-uniform 


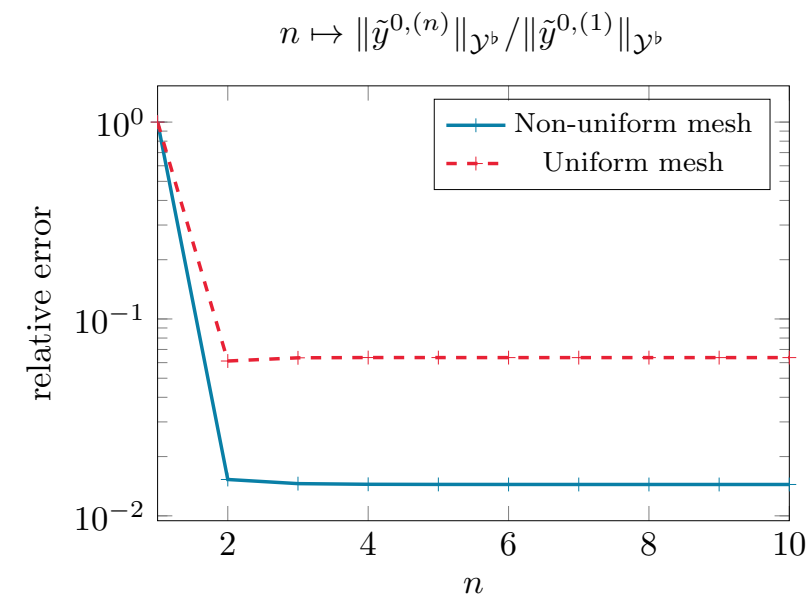

FigURE $9 . \log _{10}$ of the relative error versus the number of iterations in the case of noisy observations data.
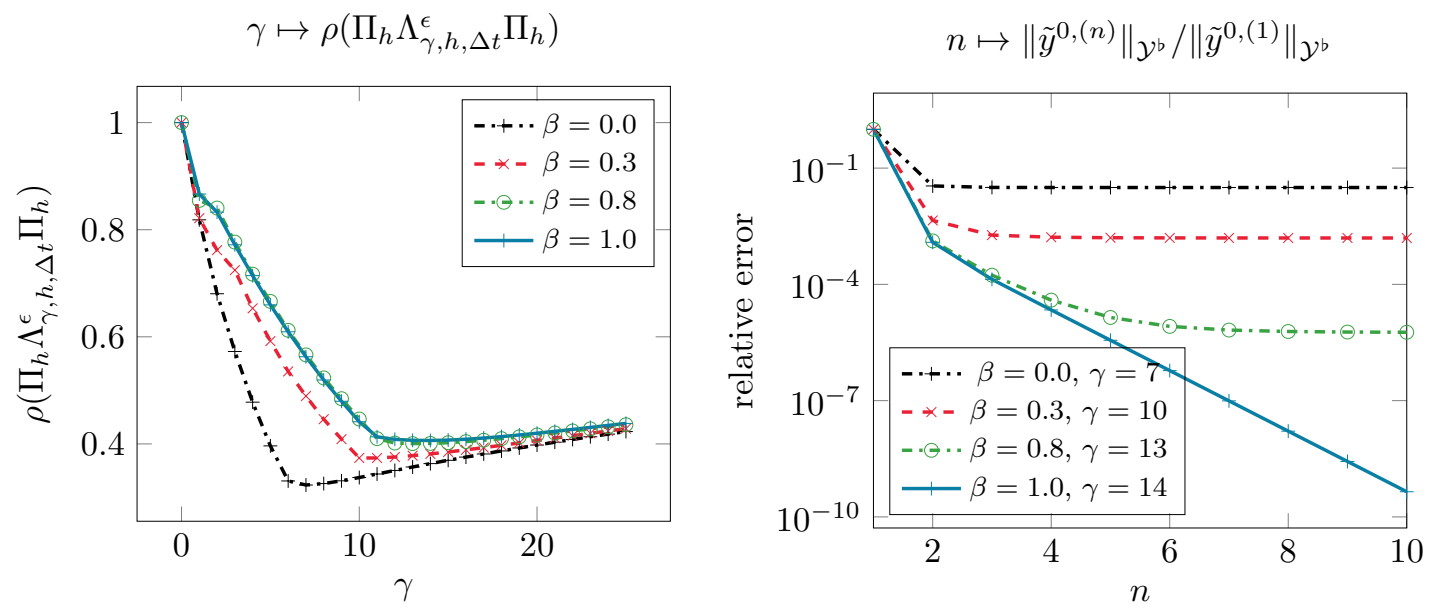

FiguRE 10. Impact of the approximate boundary condition: (left) norm of the reconstruction operator; (right) $\log _{10}$ of the relative error versus the number of iterations taking in each case the best value of parameter $\gamma$.

mesh to ensure convergence). However, we see on the convergence results that the induced consistency error is rather high. In fact, the better the approximate boundary condition is, the better the convergence of the reconstruction algorithm will be.

\subsection{D examples}

We can now show some illustrative examples in the 2D cases envisioned in Figure 2, namely the 2D unbounded case and the waveguide. We stress here that the form of the algorithm $\left(\mathrm{Ad}_{h}^{b} \& \mathrm{~F}_{h}^{b}+\operatorname{Proj}\right)$ is still valid but with $2 \mathrm{D}$ finite element operators.

\subsubsection{The $2 D$ unbounded example}

We consider a 2D case unbounded in every direction. The bounded domain is then a disc of radius 1 , discretized with triangular elements as presented in Figure 11(a). 


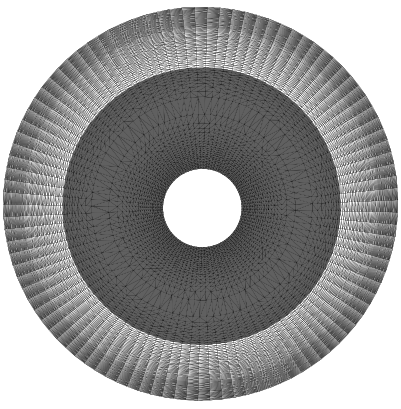

(a)

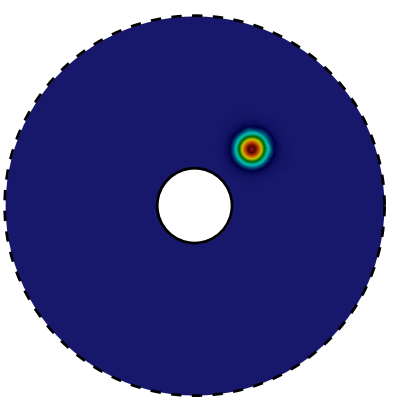

(b)

Figure 11. Circle configuration $\mathcal{D}=\mathbb{R}^{2} \backslash\left\{x^{2}+y^{2} \leq 0.2\right\}$ : (a) Mesh of the bounded domain $\mathcal{D}_{b}=\left\{x^{2}+y^{2} \leq 1\right\} \backslash\left\{x^{2}+y^{2} \leq 0.2\right\}$. (b) Target initial condition to be reconstructed.

We consider Dirichlet boundary coniditions on the boundary of the inner circle. The initial condition is represented in Figure 11(b) and is given by

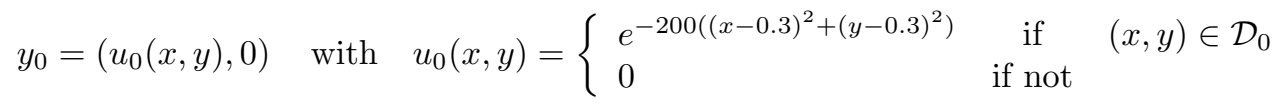

where $\mathcal{D}_{0}=\left\{0.2<\sqrt{x^{2}+y^{2}}<0.7\right\}$. The observation zone is given by $\mathcal{D}_{\text {obs }}=\left\{0.7 \leq \sqrt{x^{2}+y^{2}} \leq 0.9\right\}$. We generate the data using a very large domain. However in the observer, we consider two types of approximate transparent boundary conditions, namely of the form

$$
\nabla u \cdot n+\delta u=-\partial_{t} u \quad \text { in } \quad[0, T] \times \partial \mathcal{D}_{\text {int }} .
$$

with $\delta=0$ or $\delta=\frac{1}{2}$ for our disc example. The case $\delta=0$ illustrates the first part of our proof of Proposition 5.3. The case $\delta=\frac{1}{2}$ is known to be a more accurate choice of approximate transparent boundary conditions in general [20]. In Figure 15, we represent snapshots of the state field of the observers, using $\delta=\frac{1}{2}$, at different times during the first iteration of the algorithm. To get the backward observer, we simply use $\hat{y}_{b, h}(t)=\hat{y}_{h}(t)+2 q_{h}(t)$. We can see in this example that we approximately recover the exact initial state after one iteration. This is quickly improved after a few iterations as we can see in Figure 12 showing the reconstructed state field at different iterations of the algorithm. Moreover, the reconstruction has similar performance for the two choices of boundary conditions, which we believe is due to the fact that we rely on measurements in the neighbourhood of the boundary, hence mitigating the consistency error introduced by the approximate transparent boundary conditions.

\subsubsection{The waveguide example}

We consider a waveguide geometry with an observation zone presented in Figure 13. The depicted target initial condition is given by

$$
y_{0}=\left(u_{0}(x, y), 0\right) \quad \text { with } \quad u_{0}(x, y)=\left\{\begin{array}{ll}
y e^{-(20(x-0.5))^{2}} & \text { if } \\
0 & \text { if not }
\end{array} \quad(x, y) \in \mathcal{D}_{0}\right.
$$

where $\mathcal{D}_{0}=[-0.2,1.2] \times[0,1]$ and $\mathcal{D}_{b}=[-0.25,1.25] \times[0,1]$. Moreover, we consider approximate transparent boundary condition for the observers, i.e. $\partial_{n} u=-\partial_{t} u$. The initial condition of observer is pictured in Figure 13 (left) and given by

$$
\hat{y}_{0}=\left(\hat{u}_{0}(x, y), 0\right) \quad \text { with } \quad \hat{u}_{0}(x, y)=\left\{\begin{array}{ll}
e^{-40\left((x-0.75)^{2}+(x-0.25)^{2}\right)} & \text { if } \\
0 & \text { if not }
\end{array} \quad(x, y) \in \mathcal{D}_{0}\right.
$$



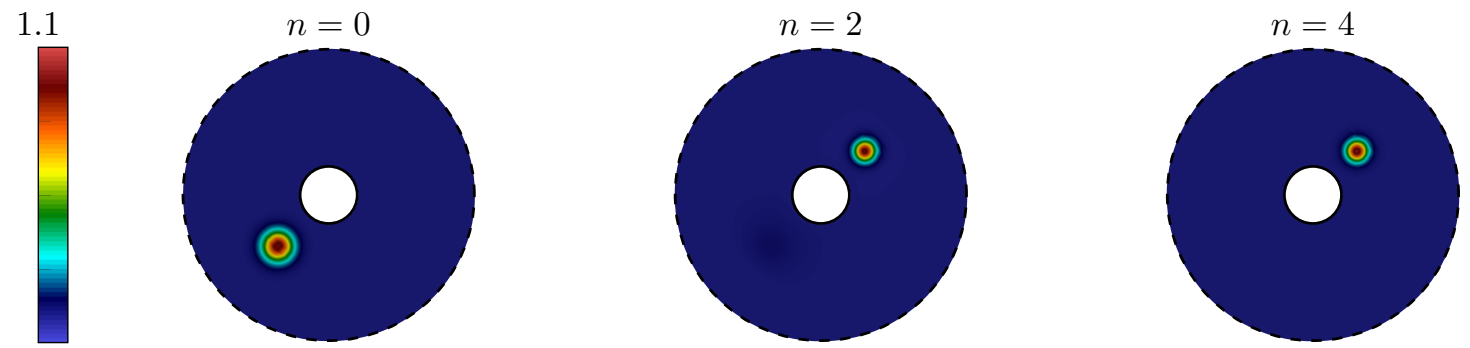

$-0.1$
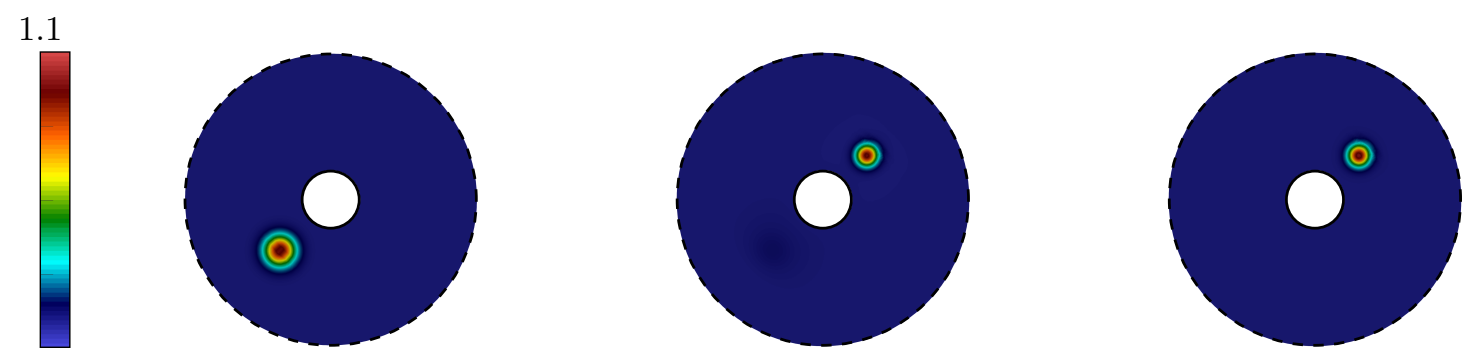

Figure 12. Initial state reconstruction at iteration $\{0,2,4\}$ : (top) using $\delta=\frac{1}{2}$; (bottom) using using $\delta=0$.

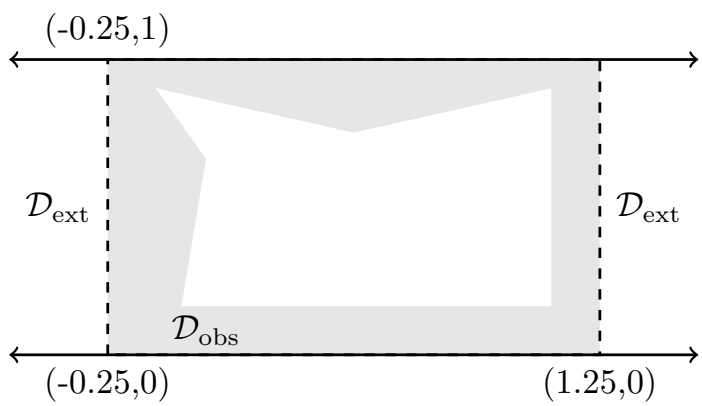

(a)

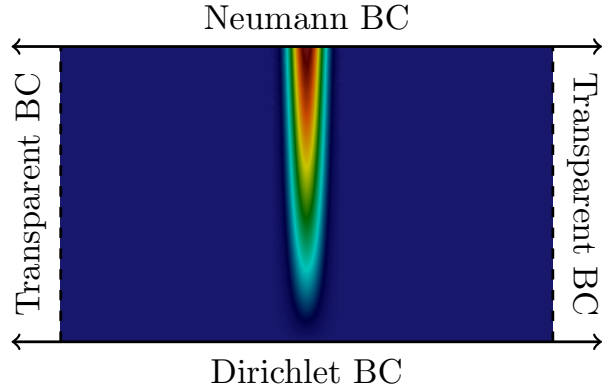

(b)

FiguRE 13. Waveguide configuration $\mathcal{D}=\mathbb{R} \times[0,1]$ : (a) Observation region $\mathcal{D}_{\text {obs }}$ on grey in a $2 \mathrm{D}$ waveguide. (b) Target initial condition to be reconstructed

We represent in Figure 16 some snapshots of the state field at different time during the first iteration of the algorithm. As previously, to get the backward observer, we simply use $\hat{y}_{b, h}(t)=\hat{y}_{h}(t)-2 q_{h}(t)$. As we can see, starting from a completely wrong initial field, we recover a very good approximation of the initial state field after one iteration. Now, in Figure 14, we have represented the initial state reconstruction. As we see, within a few iterations the convergence is achieved. Note that in that case, since we do not use exact $\mathrm{BC}$ for the observers we get a consistency error.

\subsubsection{A prospective example}

We would like to finish this article by a perspective example showing the potential of our approach. We still consider a waveguide example recalling the motivating example Figure 1. However we consider transparent boundary conditions for the observers based on [28], Neumann boundary conditions on the bottom of the waveguide and an inclusion. Let us remark that since we consider Neumann boundary conditions everywhere and transparent boundary condition, this example generalize our framework. In Figure 17, we have represented snapshots of the state field at different time during the first iteration of the 


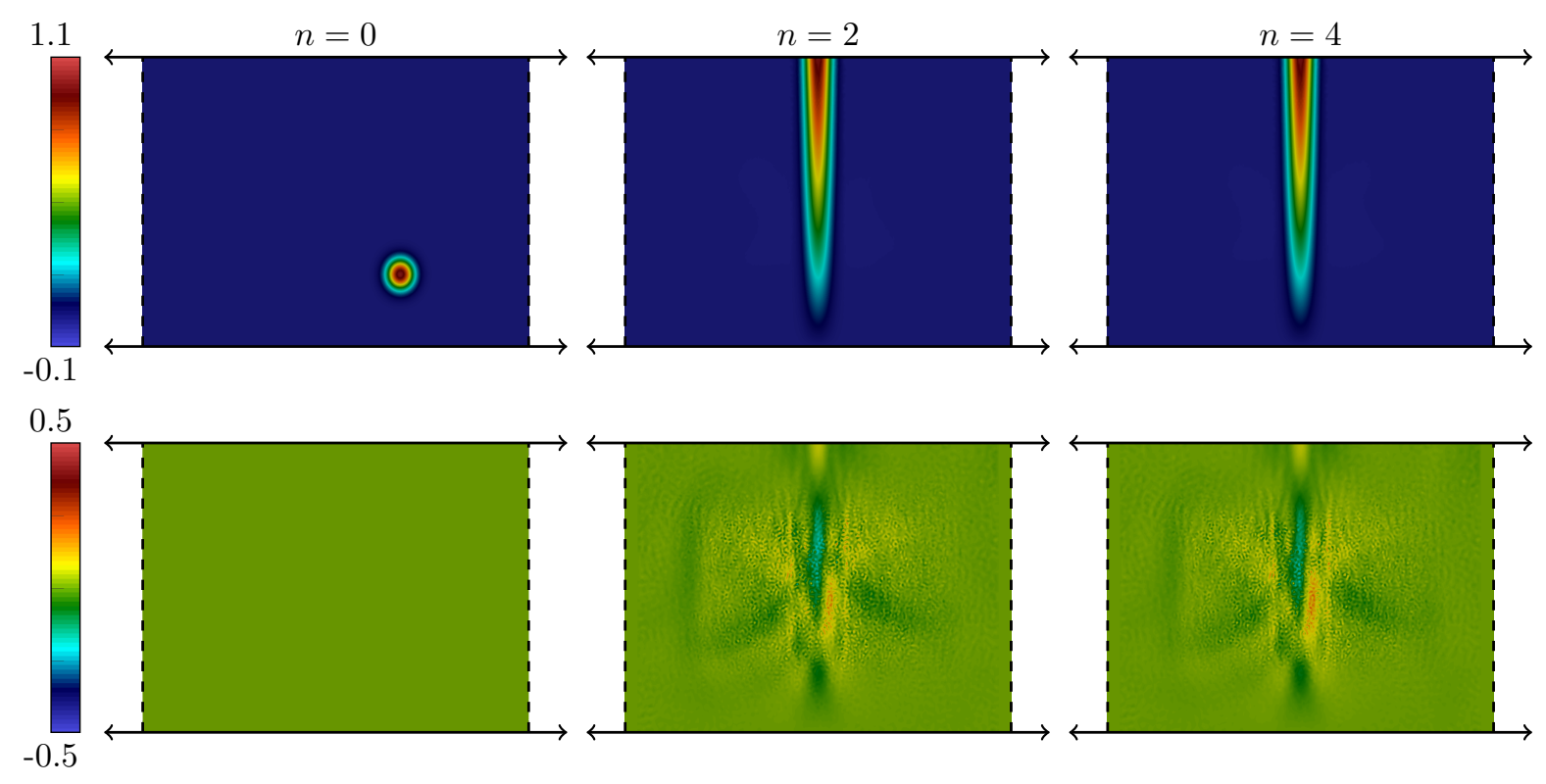

FiguRE 14. Initial state reconstruction at iteration $\{0,2,4\}$ : (top) state field from left to right; (bottom) velocity state from left to right.

algorithm. Once again, starting from a wrong initial field, we recover a very good approximation of the initial state field after one iteration. 


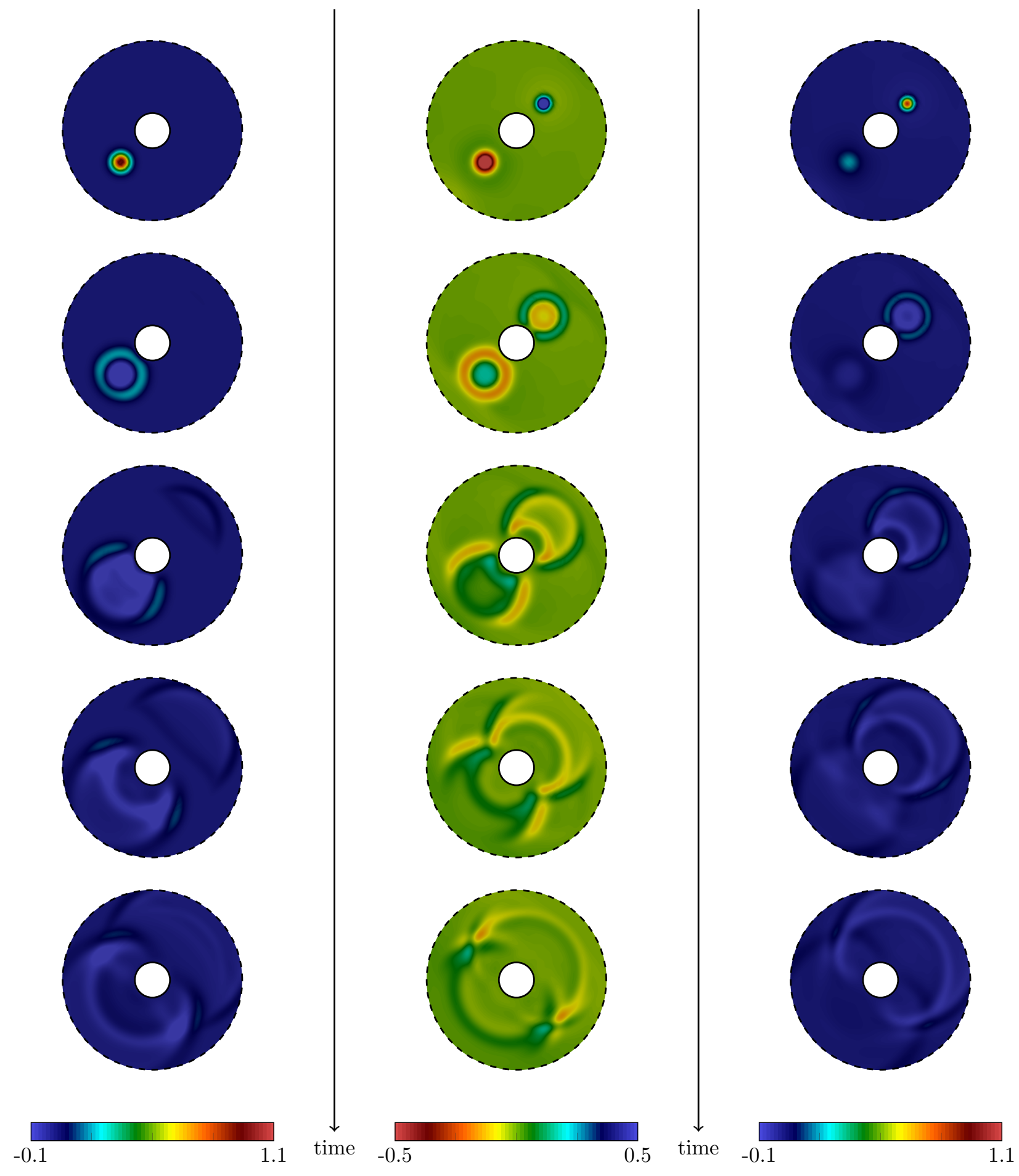

FIgURE 15. Snapshots of the observers at different times during the first iteration of the algorithm: (left) the Forward observer from top to bottom; (center) Adjoint observer from bottom to top; (right) Backward observer from bottom to top 

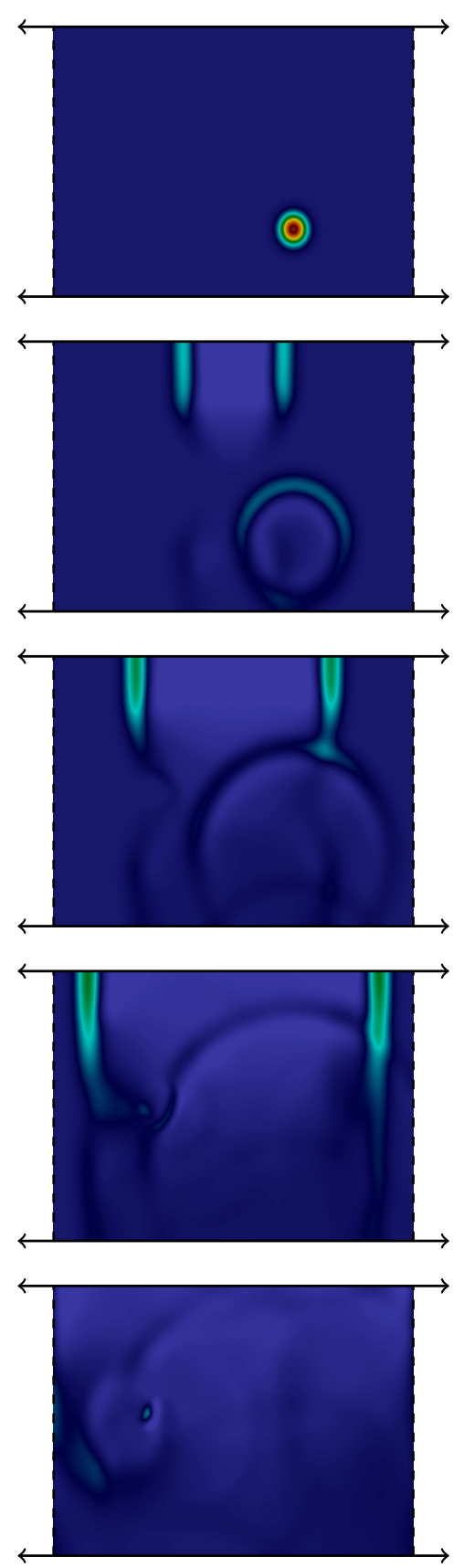

$-0.1$
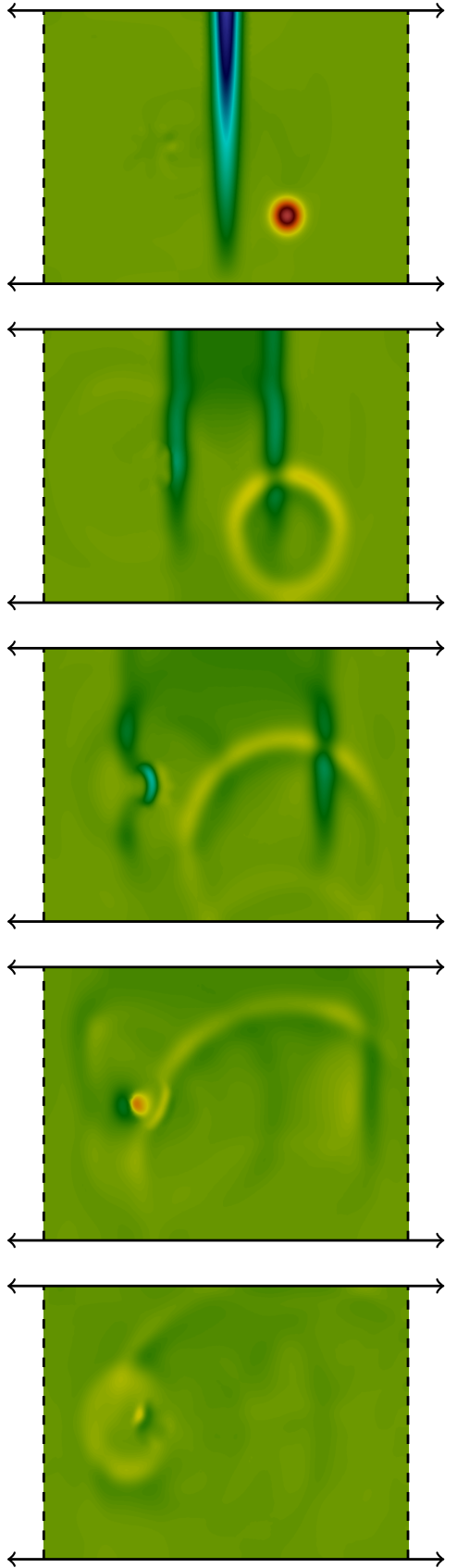

$-0.5$
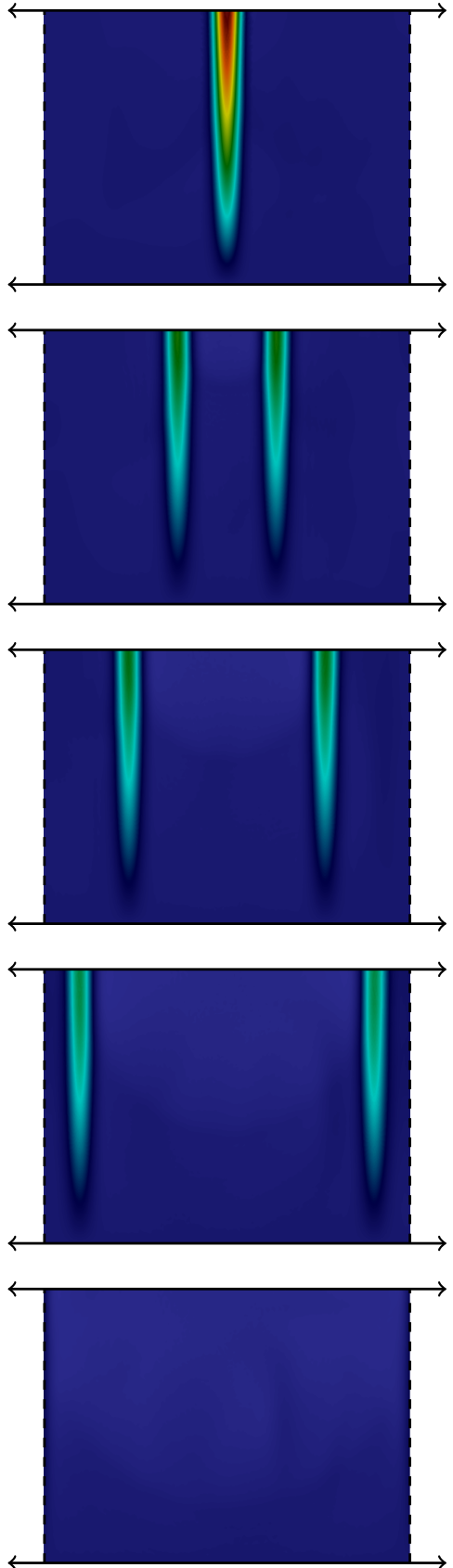

$-0.1$

Figure 16. Snapshots of the observers at different times during the first iteration of the algorithm: (left) the Forward observer from top to bottom; (center) Adjoint observer from bottom to top; (right) Backward observer from bottom to top 

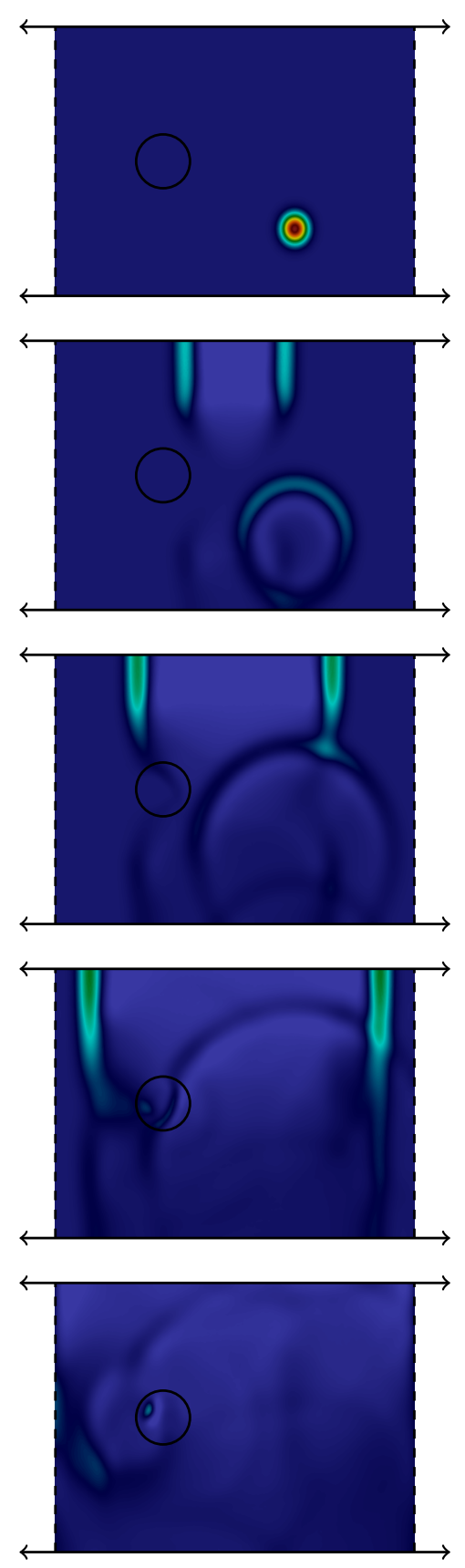

$-0.1$
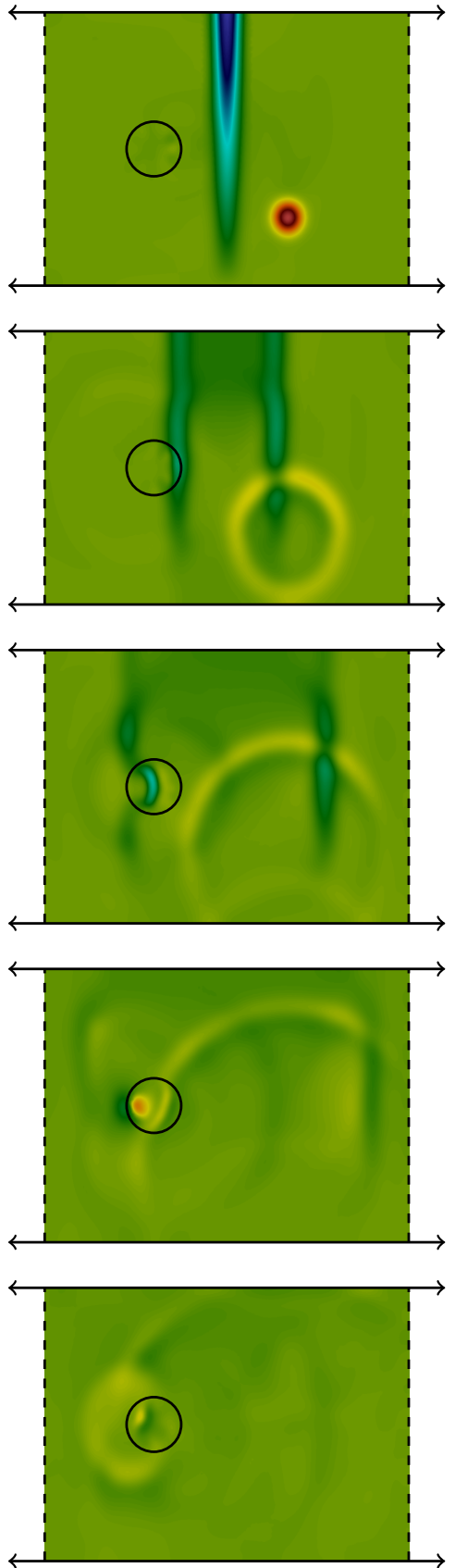

$-0.5$
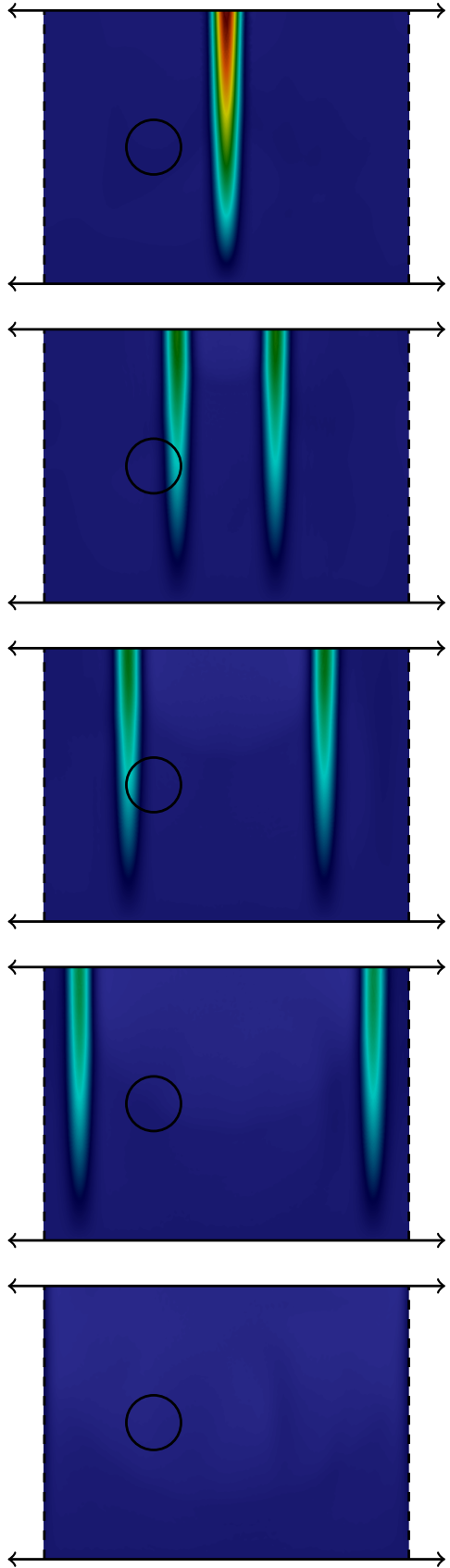

$-0.1$

Figure 17. Snapshots of the observers at different times during the first iteration of the algorithm: (left) the Forward observer from top to bottom; (center) Adjoint observer from bottom to top; (right) Backward observer from bottom to top. The black circle represents the position of the inclusion 


\section{Appendix A. An ENERGy EQUIREPARTITION RESUlt}

Theorem A.1. For all $T>0$, there exists $\kappa>0$ such that all functions

$$
u \in \mathrm{C}^{2}\left([0, T] ; \mathrm{L}^{2}(\Omega)\right) \cap \mathrm{C}^{1}\left([0, T] ; \mathrm{H}^{1}(\Omega)\right),
$$

solution of

$$
\partial_{t}^{2} u-\Delta u=0, \quad \text { in } \mathcal{D}_{\text {int }} \backslash \overline{\mathcal{O}}, \quad u=0 \text { on } \partial \mathcal{O} \times[0, T]
$$

with $\left(u(0), \partial_{t} u(0)\right)$ supported in $\mathcal{D}_{0}$ satisfy

$$
\int_{0}^{T}\|\nabla u(t)\|_{\mathrm{L}^{2}\left(\mathcal{D}_{c}\right)}^{2} \mathrm{~d} t \leq \kappa \int_{0}^{T}\left\|\partial_{t} u(t)\right\|_{\mathrm{L}^{2}\left(\mathcal{D}_{o b s}\right)}^{2} \mathrm{~d} t .
$$

Proof. Some of the elements of the proof are inspired from [13]. We use a specific multiplier in (89). Considering the test function $v=\xi_{c} u$ (we recall that $\xi_{c}$ is defined in (39)), we have

$$
\left(\partial_{t}^{2} u, \xi_{c} u\right)_{\mathrm{L}^{2}(\mathcal{D})}+\left(\nabla u, \nabla\left(\xi_{c} u\right)\right)_{\mathrm{L}^{2}(\mathcal{D})}=0 .
$$

After integration in time over $[0, T]$, and using Green's formula, in space we obtain

$$
\int_{0}^{T}\left[\left(\nabla u, \xi_{c} \nabla u\right)_{\mathrm{L}^{2}(\mathcal{D})}-\left(\partial_{t} u, \xi_{c} \partial_{t} u\right)_{\mathrm{L}^{2}(\mathcal{D})}-\frac{1}{2}\left(u,\left(\Delta \xi_{c}\right) u\right)_{\mathrm{L}^{2}(\mathcal{D})}\right] \mathrm{d} t+\left(u, \xi_{c} \partial_{t} u\right)_{\mathrm{L}^{2}(\mathcal{D})}(T)=0 .
$$

To obtain the equation above, we have used the property that, by definition, the initial data are supported only in $\mathcal{D}_{0}$ and $\xi_{c}$ vanishes in this domain. Then, as $\xi_{c}$ is non-zero only in $\mathcal{D}_{\text {obs }} \backslash \mathcal{D}_{0}$, we deduce that for any given $T>0$ there exists $\kappa$ such that,

$$
\begin{aligned}
\int_{0}^{T}\left\|\sqrt{\xi_{c}} \nabla u(t)\right\|_{\mathrm{L}^{2}(\mathcal{D})}^{2} \mathrm{~d} t \leq \kappa \int_{0}^{T}\|u(t)\|_{\mathrm{L}^{2}\left(\mathcal{D}_{\mathrm{obs}} \backslash \mathcal{D}_{0}\right)}^{2}+\left\|\partial_{t} u(t)\right\|_{\mathrm{L}^{2}\left(\mathcal{D}_{\mathrm{obs}} \backslash \mathcal{D}_{0}\right)}^{2} \mathrm{~d} t & \\
& +\left(\|u(T)\|_{\mathrm{L}^{2}\left(\mathcal{D}_{\mathrm{obs}} \backslash \mathcal{D}_{0}\right)}^{2}+\left\|\xi_{c} \partial_{t} u(T)\right\|_{\mathrm{L}^{2}(\mathcal{D})}^{2}\right) .
\end{aligned}
$$

To finish the proof, we need to estimate the last two terms of the equation above. Since $u(0)$ vanishes in $\mathcal{D}_{\text {obs }} \backslash \mathcal{D}_{0}$ by definition, we have, for all $t \in[0, T]$

$$
\|u(t)\|_{\mathrm{L}^{2}\left(\mathcal{D}_{\mathrm{obs}} \backslash \mathcal{D}_{0}\right)}^{2} \leq t \int_{0}^{t}\left\|\partial_{t} u(s)\right\|_{\mathrm{L}^{2}\left(\mathcal{D}_{\mathrm{obs}} \backslash \mathcal{D}_{0}\right)}^{2} \mathrm{~d} s .
$$

We now need an estimation of $\left\|\xi_{c} \partial_{t} u(T)\right\|_{\mathrm{L}^{2}(\mathcal{D})}^{2}$. To obtain such an estimate, we use $v=\xi_{c}^{2} \partial_{t} u$ as a test function in (89) and we obtain

$$
\left(\partial_{t}^{2} u, \xi_{c}^{2} \partial_{t} u\right)_{\mathrm{L}^{2}(\mathcal{D})}+\left(\nabla u, \nabla\left(\xi_{c}^{2} \partial_{t} u\right)\right)_{\mathrm{L}^{2}(\mathcal{D})}=0
$$

By standard energy estimates, we can show that

$$
\left\|\xi_{c} \partial_{t} u(T)\right\|_{\mathrm{L}^{2}(\mathcal{D})}^{2}+\left\|\xi_{c} \nabla u(T)\right\|_{\mathrm{L}^{2}(\mathcal{D})}^{2}=-4 \int_{0}^{T} \int_{\mathcal{D}} \xi_{c} \nabla \xi_{c} \cdot \nabla u(t) \partial_{t} u(t) \mathrm{d} t
$$

hence using Young's inequality $2 a b \leq a^{2} / \nu^{2}+b^{2} \nu^{2}$, we find

$$
\left\|\xi_{c} \partial_{t} u(T)\right\|_{\mathrm{L}^{2}(\mathcal{D})}^{2} \leq 2 \int_{0}^{T} \frac{1}{\nu^{2}}\left\|\sqrt{\xi_{c}} \nabla u\right\|_{\mathrm{L}^{2}(\mathcal{D})}^{2}+\nu^{2}\left\|\sqrt{\xi_{c}} \nabla \xi_{c} \partial_{t} u\right\|_{\mathrm{L}^{2}(\mathcal{D})}^{2} \mathrm{~d} t .
$$


Using (91) into (90), we obtain that there exists $\tilde{\kappa}>0$ (depending on $\nu, \xi_{c}$ and $T$ ) such that

$$
\left(1-\frac{2}{\nu^{2}}\right) \int_{0}^{T}\left\|\sqrt{\xi_{c}} \nabla u(t)\right\|_{\mathrm{L}^{2}(\mathcal{D})}^{2} \mathrm{~d} t \leq \tilde{\kappa} \int_{0}^{T}\left\|\partial_{t} u(t)\right\|_{\mathrm{L}^{2}\left(\mathcal{D}_{\mathrm{obs}} \backslash \mathcal{D}_{0}\right)}^{2} \mathrm{~d} t \leq \tilde{\kappa} \int_{0}^{T}\left\|\partial_{t} u(t)\right\|_{\mathrm{L}^{2}\left(\mathcal{D}_{\mathrm{obs}}\right)}^{2} \mathrm{~d} t .
$$

We end the proof taking $\nu^{2}=4$ giving finally

$$
\left(1-\frac{2}{\nu^{2}}\right) \int_{0}^{T}\left\|\sqrt{\xi_{c}} \nabla u(t)\right\|_{\mathrm{L}^{2}(\mathcal{D})}^{2} \mathrm{~d} t \geq \frac{1}{2} \int_{0}^{T}\|\nabla u(t)\|_{\mathrm{L}^{2}\left(\mathcal{D}_{c}\right)}^{2} \mathrm{~d} t .
$$

\section{Appendix B. Proof of Lemma 5.1}

In this appendix, we show that the operator $A^{b}$ defined in (76) is maximal dissipative. First we show that $A^{b}$ is dissipative. We have that $y^{\sharp}=P_{0} y=(u, w, \psi)$ belongs to $\mathcal{Y}^{b}$ for all $y=(u, v, \psi) \in D\left(A^{b}\right)$. Then, using the definition of the scalar product on $V$ one can show that

$$
\left(A^{b} y, y\right)_{\mathcal{Y}^{b}}=\left(A^{\sharp} P_{0} y, P_{0} y\right)_{\mathcal{Y}^{b}}=\left(A^{\sharp} y^{\sharp}, y^{\sharp}\right)_{\mathcal{Y}^{b}}=-\beta\left\|Q_{0}^{*} w\right\|_{\mathcal{U}}^{2}=-\beta\left\|B_{0}^{*} v\right\|_{\mathcal{U}}^{2},
$$

hence $A^{b}$ is dissipative. Then we need to show that for all $\lambda>0, \lambda \mathbb{1}-A^{b}$ is surjective from $D\left(A^{b}\right)$ into $\mathcal{Y}^{b}$. That is to say, for any $f=\left(f_{u}, f_{v}, f_{\psi}\right) \in \mathcal{Y}^{\mathrm{b}}$, we look for a solution $y=(u, v, \psi) \in D\left(A^{b}\right)$ of the problem

$$
\left(\lambda \mathbb{1}-A^{b}\right) y=f .
$$

If a solution exists then

therefore $u$ should satisfy

$$
\lambda u-v=f_{u} \quad \text { and } \quad \lambda \psi-\sqrt{\delta} B_{0}^{*} v=f_{\psi},
$$

$$
\lambda v-A_{0}^{b} u-\beta B_{0} B_{0}^{*} v-\sqrt{\delta} B_{0} \psi=f_{v},
$$

i.e.

$$
\left(\lambda^{2} \mathbb{1}+A_{0}^{b}+(\lambda \beta+\delta) B_{0} B_{0}^{*}\right) u=\lambda f_{u}+\left(\beta+\frac{\delta}{\lambda}\right) B_{0} B_{0}^{*} f_{u}-f_{v}-\frac{\sqrt{\delta}}{\lambda} B_{0} f_{\psi} .
$$

One can check that the right-hand side of the equation above belongs to $\mathcal{V}^{\prime}$. Therefore $u$ must satisfy the following variational problem: for any $\tilde{u} \in \mathcal{V}$,

$$
\lambda^{2}(u, \tilde{u})_{\mathcal{H}}+(u, \tilde{u})_{\mathcal{V}}+(\lambda \beta+\delta)\left(B_{0}^{*} u, B_{0}^{*} \tilde{u}\right)_{\mathcal{U}}=\ell(\tilde{u}),
$$

where $\ell(\cdot)$ is a linear form on $\mathcal{V}$ that depends on $f$. Equation (97) defines a well-posed problem and by a direct application of Lax-Milgram theorem one can show that there exists a unique solution $u \in \mathcal{V}$ for all $\lambda>0$. Then one can define $v \in \mathcal{V}$ and $\psi \in \mathcal{U}$ using (94). To finish the proof we need to show that (95) is, indeed, satisfied. Note that by construction of $u$ from the variational formulation (97) equation (96) is satisfied in $\mathcal{V}^{\prime}$ and therefore (95) is satisfied in $\mathcal{V}^{\prime}$. Moreover, we have

$$
A_{0}^{b} u+\beta B_{0} B_{0}^{*} v+\sqrt{\delta} B_{0} \psi=\lambda v-f_{v} \in \mathcal{H},
$$

hence, we have shown that (93) is satisfied with $y \in D\left(A^{b}\right)$.

\section{Appendix C. Proof of an observation inequality By multipliers}

From the assumptions on the geometry made in Section 2.1, we can define two disjoint boundaries $\widetilde{\Gamma}$ and $\widetilde{\Sigma}$ as

$$
\widetilde{\Sigma}=\partial \mathcal{O}, \quad \widetilde{\Gamma}=\partial \mathcal{D}_{\text {int }} .
$$


Note that, thanks to our domain assumptions $\widetilde{\Gamma}$ and $\widetilde{\Sigma}$ are well-separated in the sense that the distance between these two boundaries is bounded by below. Moreover, for the sake of conciseness, we denote

$$
\Omega=\mathcal{D}_{\text {int }} \backslash \overline{\mathcal{O}}, \quad \Omega_{T}=\Omega \times(0, T), \quad \widetilde{\Sigma}_{T}=\widetilde{\Sigma} \times(0, T) .
$$

We give a preliminary lemma which is a standard result in multiplier theory (see Chapter 7.1 of [52] and therefore its proof is just sketched.

Lemma C.1. For any $g \in \mathrm{W}^{1, \infty}(\Omega), h \in\left[\mathrm{W}^{1, \infty}(\Omega)\right]^{d}$ vanishing along $\widetilde{\Gamma}$ and

$$
u \in \mathrm{C}^{2}\left([0, T] ; \mathrm{L}^{2}(\Omega)\right) \cap \mathrm{C}^{1}\left([0, T] ; \mathrm{H}^{1}(\Omega)\right), \quad \Delta u \in \mathrm{C}^{0}\left([0, T] ; \mathrm{L}^{2}(\Omega)\right), \quad u=0 \text { on } \widetilde{\Sigma}_{T}
$$

then

$$
\begin{aligned}
\int_{\Omega_{T}} \partial_{t}^{2} u(g u+h \cdot \nabla u) \mathrm{d} \mathbf{x} \mathrm{d} t=\int_{\Omega_{T}}\left(\frac{\nabla \cdot h}{2}-g\right)\left|\partial_{t} u\right|^{2} \mathrm{~d} \mathbf{x} \mathrm{d} t & \\
& +\left[\frac{1}{2} \frac{d}{d t} \int_{\Omega} g|u|^{2} \mathrm{~d} \mathbf{x}+\int_{\Omega} \nabla u \cdot h \partial_{t} u \mathrm{~d} \mathbf{x}\right]_{0}^{T},
\end{aligned}
$$

and

$$
\begin{aligned}
-\int_{\Omega_{T}} \Delta u(g u+h \cdot \nabla u) \mathrm{d} \mathbf{x} \mathrm{d} t= & \\
\int_{\Omega_{T}}\left(g-\frac{\nabla \cdot h}{2}\right)|\nabla u|^{2} \mathrm{~d} \mathbf{x} \mathrm{d} t+ & \int_{\Omega_{T}} u \nabla g \cdot \nabla u \mathrm{~d} \mathbf{x} \mathrm{d} t+\sum_{i, j=1}^{d} \int_{\Omega_{T}} \partial_{x_{i}} u \partial_{x_{j}} u \partial_{x_{i}} h_{j} \mathrm{~d} \mathbf{x} \mathrm{d} t \\
& +\frac{1}{2} \int_{\widetilde{\Sigma}_{T}}(h \cdot n)|\nabla u|^{2} \mathrm{~d} \gamma \mathrm{d} t-\int_{\widetilde{\Sigma}_{T}}(\nabla u \cdot n) h \cdot \nabla u \mathrm{~d} \gamma \mathrm{d} t .
\end{aligned}
$$

Proof. First, let us observe that the expression above makes sense (indeed in (99) it is not clear that the boundary integrals are well defined). Since $\Delta u \in \mathrm{C}^{0}\left([0, T] ; \mathrm{L}^{2}(\Omega)\right)$ we have

$$
\nabla u \in \mathrm{C}^{0}([0, T] ; H(\operatorname{div}, \Omega) \cap H(\operatorname{rot}, \Omega))
$$

Moreover, by assumption we also have that $\nabla u \times n=0$ along $\widetilde{\Sigma}$. Therefore, one can observe that

$$
g \nabla u(t) \in H(\operatorname{div}, \Omega) \cap H(\operatorname{rot}, \Omega), \quad g \nabla u(t) \times n=0 \text { on } \partial \Omega,
$$

hence, $g \nabla u(t) \in H^{1 / 2+s}(\Omega)^{d}$ for some $s>0$ (see for instance [44] Theorem 3.50). This implies (see [41] Theorem 3.38), $g \nabla u(t) \in \mathrm{L}^{2}(\partial \Omega)^{d}$, hence, by choosing $g$ bounded from below on $\widetilde{\Sigma}$ (this is possible since $\widetilde{\Gamma}$ and $\widetilde{\Sigma}$ are well-separated) we find that for all time $t \in[0, T]$

$$
\left.\nabla u(t)\right|_{\widetilde{\Sigma}} \in \mathrm{L}^{2}(\widetilde{\Sigma})
$$

Note that we have used the assumption that $\Omega$ is a Lipschitz bounded domain and $\mathcal{D}_{\text {int }}$ is convex which are consequences of the geometry assumptions of Section 2.1. Finally by standard computations using Green's formula on can show that (98) and (99) hold.

Theorem C.2. Assume $\widetilde{\Sigma}_{T} \neq \emptyset$. There exists $\kappa>0$ (not depending on $T$ ) such that all functions

$$
u \in \mathrm{C}^{2}\left([0, T] ; \mathrm{L}^{2}(\Omega)\right) \cap \mathrm{C}^{1}\left([0, T] ; \mathrm{H}^{1}(\Omega)\right)
$$

solution of

$$
\partial_{t}^{2} u-\Delta u=0, \quad \text { in } \Omega, \quad u=0 \text { on } \widetilde{\Sigma}_{T}
$$


satisfy

$$
\frac{T}{2} \mathcal{E}(0)-\kappa(\mathcal{E}(T)+\mathcal{E}(0)) \leq \tilde{\kappa}(T) \int_{\mathcal{D}_{c} \times(0, T)}\left|\partial_{t} u\right|^{2}+|\nabla u|^{2} \mathrm{~d} \mathbf{x} \mathrm{d} t
$$

where $\tilde{\kappa}(T)>0$ depends on $T$ and where the energy $\mathcal{E}(t) \in \mathrm{C}^{1}([0, T])$ is given by

$$
\mathcal{E}(t)=\left\|\partial_{t} u(t)\right\|_{\mathrm{L}^{2}(\Omega)}^{2}+\|\nabla u(t)\|_{\mathrm{L}^{2}(\Omega)}^{2} .
$$

Proof. By assumption $u$ is sufficiently regular, and satisfies the homogeneous Dirichlet boundary conditions along $\widetilde{\Sigma}_{T}$, so that Lemma C.1 can be used. We also have

$$
\int_{\Omega_{T}} \partial_{t}^{2} u v \mathrm{~d} \mathbf{x}-\int_{\Omega_{T}} \Delta u v \mathrm{~d} \mathbf{x}=0
$$

for any test function $v$ with adequate regularity. For a given $\mathbf{x}_{0} \in \Omega$ we set $v=g u+h \cdot \nabla u$ with

$$
h(\mathbf{x})=\eta_{c}(\mathbf{x})\left(\mathbf{x}-\mathbf{x}_{0}\right) \quad \text { and } \quad g(\mathbf{x})=\frac{d-1}{2} \eta_{c}(\mathbf{x}),
$$

where $\eta_{c}(\mathbf{x})=1-\chi_{c}(\mathbf{x})$, with $\chi_{c}$ defined by (38). The function $\eta_{c}$ is a positive smooth function such that $\nabla \eta_{c}=0$ in $\Omega \backslash \mathcal{D}_{c}$ and $\eta_{c}$ vanishes along $\widetilde{\Gamma}$ and equals 1 in some interior of $\Omega$ that we denote $\Omega_{\text {int }}$ and that satisfies

$$
\bar{\Omega}_{\text {int }} \cup \overline{\mathcal{D}}_{\text {obs }}=\bar{\Omega}, \quad \partial \Omega_{\text {int }} \cap \widetilde{\Sigma}=\emptyset .
$$

By definition $h$ and $g$ satisfy the assumptions of Lemma C.1 and we have

$$
\nabla \cdot h(\mathbf{x})=\eta_{c}(\mathbf{x}) d+\nabla \eta_{c}(\mathbf{x}) \cdot\left(\mathbf{x}-\mathbf{x}_{0}\right), \quad \partial_{x_{i}} h_{j}(\mathbf{x})=0, \quad i \neq j, \text { in } \Omega \backslash \mathcal{D}_{c} .
$$

Using (101) and lemma C.1 we can show that

$$
\begin{gathered}
0=\frac{1}{2} \int_{\Omega_{\mathrm{int}} \times(0, T)}\left|\partial_{t} u\right|^{2}+|\nabla u|^{2} \mathrm{~d} \mathbf{x} \mathrm{d} t \\
+\int_{\mathcal{D}_{c} \times(0, T)}\left(\frac{\nabla \cdot h}{2}-g\right)\left(\left|\partial_{t} u\right|^{2}-|\nabla u|^{2}\right) \mathrm{d} \mathbf{x} \mathrm{d} t \\
\quad+\int_{\mathcal{D}_{c} \times(0, T)} u \nabla g \cdot \nabla u \mathrm{~d} \mathbf{x} \mathrm{d} t \\
+\sum_{i, j=1}^{d} \int_{\mathcal{D}_{c} \times(0, T)} \partial_{x_{i}} u \partial_{x_{j}} u \partial_{x_{i}} h_{j} \mathrm{~d} \mathbf{x} \mathrm{d} t \\
\quad+\frac{1}{2} \int_{\widetilde{\Sigma}_{T}}(h \cdot n)|\nabla u|^{2} \mathrm{~d} \gamma \mathrm{d} t \\
\quad-\int_{\widetilde{\Sigma}_{T}}(\nabla u \cdot n) h \cdot \nabla u \mathrm{~d} \gamma \mathrm{d} t \\
+\left[\frac{1}{2} \frac{d}{d t} \int_{\Omega} g|u|^{2} \mathrm{~d} \mathbf{x}+\int_{\Omega} \nabla u \cdot h \partial_{t} u \mathrm{~d} \mathbf{x}\right]_{0}^{T} .
\end{gathered}
$$

Since $u=0$ along $\widetilde{\Sigma}_{T}$ (Homogeneous Dirichlet boundary condition) we have $\nabla u \times n=0$, and therefore the boundary integrals in the equation above are equal to

$$
-\frac{1}{2} \int_{\widetilde{\Sigma}_{T}}\left(\mathbf{x}-\mathbf{x}_{0}\right) \cdot n|\nabla u \cdot n|^{2} \mathrm{~d} \gamma \mathrm{d} t
$$


Since $\mathcal{O}$ is star-shaped, there exists $\mathbf{x}_{0} \in \mathcal{O}$ such that $\left(\mathbf{x}-\mathbf{x}_{0}\right) \cdot n \leq 0$ on $\widetilde{\Sigma}$ and the integral in (102) is positive. Moreover, since $u$ vanishes at the initial time in $\mathcal{D}_{c}$ we have

$$
\|u(t)\|_{\mathrm{L}^{2}\left(\mathcal{D}_{c}\right)} \leq \int_{0}^{t}\left\|\partial_{t} u(s)\right\|_{\mathrm{L}^{2}\left(\mathcal{D}_{c}\right)} \mathrm{d} s \quad \Rightarrow \quad \int_{0}^{T}\|u(t)\|_{\mathrm{L}^{2}\left(\mathcal{D}_{c}\right)}^{2} \mathrm{~d} t \leq \frac{T^{2}}{2} \int_{0}^{T}\left\|\partial_{t} u(t)\right\|_{\mathrm{L}^{2}\left(\mathcal{D}_{c}\right)} \mathrm{d} t,
$$

and therefore every integral term in $\mathcal{D}_{c}$ can be bounded by the $\mathrm{L}^{2}$ space-time norm of $\partial_{t} u$ and $\nabla u$. It can be shown that there exists a constant $\alpha$ depending only on $\left(\mathbf{x}_{0}, \chi_{c}, T\right)$ such that

$$
\begin{aligned}
\frac{1}{2} \int_{\Omega_{\mathrm{int}} \times(0, T)}\left|\partial_{t} u\right|^{2}+|\nabla u|^{2} \mathrm{~d} \mathbf{x} \mathrm{d} t+\left[\frac{1}{2} \frac{d}{d t} \int_{\Omega} g|u|^{2} \mathrm{~d} \mathbf{x}+\int_{\Omega} \nabla u \cdot h \partial_{t} u \mathrm{~d} \mathbf{x}\right]_{0}^{T} & \\
& \leq \alpha \int_{\mathcal{D}_{c} \times(0, T)}\left|\partial_{t} u\right|^{2}+|\nabla u|^{2} \mathrm{~d} \mathbf{x} \mathrm{d} t,
\end{aligned}
$$

hence

$$
\frac{1}{2} \int_{0}^{T} \mathcal{E}_{\eta}(t) \mathrm{d} t+\left[\frac{1}{2} \frac{d}{d t} \int_{\Omega} g|u|^{2} \mathrm{~d} \mathbf{x}+\int_{\Omega} \nabla u \cdot h \partial_{t} u \mathrm{~d} \mathbf{x}\right]_{0}^{T} \leq\left(\alpha+\frac{1}{2}\right) \int_{\mathcal{D}_{c} \times(0, T)}\left|\partial_{t} u\right|^{2}+|\nabla u|^{2} \mathrm{~d} \mathbf{x} \mathrm{d} t
$$

where we have defined the energy $\mathcal{E}_{\eta}(t)$ as

$$
\mathcal{E}_{\eta}(t)=\left\|\partial_{t} u(t)\right\|_{\mathrm{L}^{2}\left(\Omega_{\mathrm{int}}\right)}^{2}+\|\nabla u(t)\|_{\mathrm{L}^{2}\left(\Omega_{\mathrm{int}}\right)}^{2}+\left\|\sqrt{\eta} \partial_{t} u(t)\right\|_{\mathrm{L}^{2}\left(\mathcal{D}_{c}\right)}^{2}+\|\sqrt{\eta} \nabla u(t)\|_{\mathrm{L}^{2}\left(\mathcal{D}_{c}\right)}^{2} \leq \mathcal{E}(t) .
$$

Additionally, one can prove that there exists another constant $\tilde{\alpha}$ depending only on $\left(\mathbf{x}_{0}, \chi_{c}\right)$ and the domain $\Omega$ (note that because of the homogeneous boundary Dirichlet condition along $\widetilde{\Sigma}$ we can use the Poincaré estimate) such that

$$
\left.\left|\frac{1}{2} \frac{d}{d t} \int_{\Omega} g\right| u(t)\right|^{2} \mathrm{~d} \mathbf{x}+\int_{\Omega} \nabla u(t) \cdot h \partial_{t} u(t) \mathrm{d} \mathbf{x} \mid \leq \tilde{\alpha} \mathcal{E}_{\eta}(t) .
$$

Therefore we deduce that

$$
\frac{1}{2} \int_{0}^{T} \mathcal{E}_{\eta}(t) \mathrm{d} t-\tilde{\alpha}\left(\mathcal{E}_{\eta}(T)+\mathcal{E}(0)\right) \leq\left(\alpha+\frac{1}{2}\right) \int_{\mathcal{D}_{\text {obs }} \times(0, T)}\left|\partial_{t} u\right|^{2}+|\nabla u|^{2} \mathrm{~d} \mathbf{x} \mathrm{d} t .
$$

Since the standard energy identity for the wave equation gives (the identity below is obtained by multiplying the first equation of (100) by $\left.\eta \partial_{t} u\right)$

$$
\frac{1}{2} \int_{0}^{T} \mathcal{E}_{\eta}(t) \mathrm{d} t=\frac{T}{2} \mathcal{E}(0)-\int_{0}^{T} \int_{0}^{t} \int_{\mathcal{D}_{c}} \partial_{t} u \nabla u \cdot \nabla \eta \mathrm{d} \mathbf{x} \mathrm{d} s \mathrm{~d} t
$$

we can deduce from (103) that

$$
\frac{T}{2} \mathcal{E}(0)-\tilde{\alpha}\left(\mathcal{E}_{\eta}(T)+\mathcal{E}(0)\right) \leq\left(\alpha+\frac{1}{2}\right) \int_{\mathcal{D}_{c} \times(0, T)}\left|\partial_{t} u\right|^{2}+|\nabla u|^{2} \mathrm{~d} \mathbf{x} \mathrm{d} t+\int_{0}^{T} \int_{0}^{t} \int_{\mathcal{D}_{c}} \partial_{t} u \nabla u \cdot \nabla \eta \mathrm{d} \mathbf{x} \mathrm{d} s \mathrm{~d} t
$$

The final statement of the theorem is obtained using that $\mathcal{E}_{\eta} \leq \mathcal{E}$ in the left-hand side of the previous equation, as well as a Cauchy-Schwarz inequality on the right-hand side.

The authors deeply thank Karim Ramdani for discussions about the back-and-forth observer reconstruction algorithm and Sonia Fliss for discussions about transparent boundary conditions. the authors are grateful to Mathieu Pernot for discussions about transient elastography and to Dominique Chapelle for his comments about the paper. 


\section{REFERENCES}

[1] H. Ammari, P. Garapon, F. Jouve, H. Kang, M. Lim, and S. Yu. A New Optimal Control Approach for the Reconstruction of Extended Inclusions. SIAM Journal on Control and Optimization, 51(2):1372-1394, January 2013. 2

[2] D. Auroux and J. Blum. A nudging-based data assimilation method: the Back and Forth Nudging (BFN) algorithm. Nonlinear Processes In Geophysics, 15(2):305-319, January 2008. 6

[3] G. Bal and S. Imperiale. Displacement Reconstructions in Ultrasound Elastography. SIAM Journal on Imaging Sciences, $8(2): 1070-1089,2015.2$

[4] H. T. Banks, K. Ito, and C. Wang. Exponentially stable approximations of weakly damped wave equations. In Estimation and control of distributed parameter systems (Vorau, 1990), pages 1-33. Birkhäuser, Basel, Basel, 1991. 30

[5] C. Bardos, G. Lebeau, and J. Rauch. Un exemple d'utilisation des notions de propagation pour le contrôle et la stabilisation des problèmes hyperboliques. Rendiconti del Seminario Matematico del Universita Politecnico Torino, Fascicolo speciale(Hyperbolic Equations (1987)):12-31, May 1988. 28

[6] A. Bensoussan. Filtrage optimal des systèmes linéaires. Dunod, 1971. 2, 3, 12

[7] A. Bensoussan, M.C. Delfour, G. Da Prato, and S.K. Mitter. Representation and Control of Infinite Dimensional Systems (Vol. 1). Birkhauser Verlag, Basel, Switzerland, Switzerland, 1992. 5, 6, 8, 17, 18, 24, 26

[8] J. Bercoff, M. Tanter, and M. Fink. Supersonic shear imaging: a new technique for soft tissue elasticity mapping. IEEE Trans Ultrason Ferr And Freq Control, 51(4):396-409, April 2004. 2

[9] J.-P. Berenger. A perfectly matched layer for the absorption of electromagnetic waves. J Comput Phys, 114(2):185-200, 1994. 30

[10] N. Burq and R. Joly. Exponential decay for the damped wave equation in unbounded domains. Commun. Contemp. Math., 18(6):1650012-1650027, 2016. 12, 13

[11] Nicolas Burq and Patrick Gérard. Stabilisation of wave equations on the torus with rough dampings. working paper or preprint, December 2017. 12, 13

[12] D. Chapelle, N. Cîndea, M. De Buhan, and P. Moireau. Exponential Convergence of an Observer Based on Partial Field Measurements for the Wave Equation. Mathematical Problems in Engineering, 2012:1-12, 2012. 6

[13] D. Chapelle, N. Cîndea, M. De Buhan, and P. Moireau. Exponential convergence of an observer based on partial field measurements for the wave equation. Mathematical Problems in Engineering, 2012:12, October 2012. 43

[14] D. Chapelle, N. Cîndea, and P. Moireau. Improving convergence in numerical analysis using observers The wave-like equation case. Mathematical Models and Methods in Applied Sciences (M3AS), pages 1-34, March 2012. 30, 33

[15] N. Cindea and A. Munch. A mixed formulation for the direct approximation of the control of minimal $L^{2}$-norm for linear type wave equations. Calcolo, 52(3):245-288, August 2015. 30

[16] M. De Buhan and M. Kray. A new approach to solve the inverse scattering problem for waves: combining the TRAC and the adaptive inversion methods. Inverse Problems, 29(8), August 2013. 19

[17] M. M. Doyley and K. J. Parker. Elastography: general principles and clincial applications. Ultrasound Clin, 9(1):1-11, January 2014. 2

[18] A. Eisenscher, E. Schweg-Toffler, G. Pelletier, and P. Jacquemard. La palpation échographique rythmée : Echosismographie. une nouvelle technique de différenciation des tumeurs bénignes et malignes par l'étude ultrasonore de l'élasticité tissulaire. J. Radiol., 64(4):255-261, 1983. 2

[19] H. W. Engl, M. Hanke, and A. Neubauer. Regularization of inverse problems, volume 375 of Mathematics and its Applications. Springer, 1996. 12

[20] B. Enquist and A. Majda. Absorbing boundary conditions for the numerical simulation of waves. Mathematics of Computations, 31:629 - 651, 1998. 23, 37

[21] S. Ervedoza, A. Marica, and E. Zuazua. Numerical meshes ensuring uniform observability of one-dimensional waves: construction and analysis. IMA Journal of Numerical Analysis, 36(2), 2016. 30

[22] S. Ervedoza and E. Zuazua. Uniform exponential decay for viscous damped systems. Progress in Nonlinear Differential Equations and their Applications, 78:95-112, 2009. 30

[23] S. Ervedoza and E. Zuazua. Uniformly exponentially stable approximations for a class of damped systems. Journal de Mathématiques Pures et Appliquées, 91(1):20-48, 2009. 30

[24] S. Ervedoza and E. Zuazua. Perfectly matched layers in 1-d: energy decay for continuous and semi-discrete waves. Numer Math, 109(4):597-634, 2008. 30

[25] R. Glowinski, W. Kinton, and M. F. Wheeler. A mixed finite element formulation for the boundary controllability of the wave equation. International Journal for Numerical Methods in Engineering, 27(3):623-635, 1989. 30

[26] M. J. Grote and J. B. Keller. Exact nonreflecting boundary conditions for the time dependent wave equation. SIAM Journal on Applied Mathematics, 55(2):280-297, 1995. 23

[27] T. Ha-Duong and P. Joly. On the Stability Analysis of Boundary Conditions for the Wave Equation by Energy Methods. Part I: The Homogeneous Case. Mathematics of Computation, 62(206):539-563, April 1994. 23, 24

[28] T. Hagstrom. New Results on Absorbing Layers and Radiation Boundary Conditions, pages 1-42. Springer Berlin Heidelberg, Berlin, Heidelberg, 2003. 38

[29] T. Hagstrom and S. I. Hariharan. A formulation of asymptotic and exact boundary conditions using local operators. Applied Numerical Mathematics, 27(4):403 - 416, 1998. Special Issue on Absorbing Boundary Conditions. 23

[30] G. Haine. An observer-based approach for thermoacoustic tomography. In The 21st International Symposium on Mathematical Theory of Networks and Systems, pages 1-9, 2014. 3, 6, 8 
[31] G. Haine. Recovering the observable part of the initial data of an infinite-dimensional linear system with skew-adjoint generator. Mathematics of Control Signals and Systems, 26(3):435-462, January 2014. 15

[32] G. Haine and K. Ramdani. Reconstructing initial data using observers: error analysis of the semi-discrete and fully discrete approximations. Numer Math, 120(2):307-343, 2012. 30

[33] I. Lasiecka and R. Triggiani. Exact controllability of the wave equation with neumann boundary control. Applied Mathematics and Optimization, 19(1):243-290, Jan 1989. 24

[34] I. Lasiecka and R. Triggiani. Control Theory for Partial Differential Equations: Volume 1, Abstract Parabolic Systems: Continuous and Approximation Theories. Control Theory for Partial Differential Equations: Continuous and Approximation Theories. Cambridge University Press, 2000. 24

[35] I. Lasiecka and R. Triggiani. $L_{2}(\Sigma)$-regularity of the boundary to boundary operator $B^{*} L$ for hyperbolic and Petrowski PDEs. Abstract Applied Analysis, 2003(19):1061-1139, 2003. 18, 24, 25

[36] J. Le Rousseau, G. Lebeau, P. Terpolilli, and E. Trélat. Geometric control condition for the wave equation with a timedependent observation domain. Anal. PDE, 10(4):983-1015, 2017. 28

[37] G. Lebeau, C. Bardos, and J. Rauch. Sharp Sufficient Conditions for the Observation, Control, and Stabilization of Waves from the Boundary. SIAM Journal on Control and Optimization, 30(5):1024-1065, 1992. 28

[38] J.-L. Lions. Contrôle optimal de systèmes gouvernés par des équations aux dérivées partielles. Avant propos de P. Lelong. Dunod, Paris, 1968. 2

[39] A. Marica and E. Zuazua. Propagation of 1D Waves in Regular Discrete Heterogeneous Media: A Wigner Measure Approach. Found Comput Math, 15(6):1571-1636, 2015. 30, 31, 33

[40] J. McLaughlin and D. Renzi. Shear wave speed recovery in transient elastography and supersonic imaging using propagating fronts. Inverse Problems, 22(2):681-706, March 2006. 2

[41] W. McLean. Strongly Elliptic systems and Boundary Integral equation. Cambridge University Press, Cambridge, 2000.45

[42] L. Miller. Resolvent conditions for the control of unitary groups and their approximations. J. Spectr. Theory, 2(1):1-55, 2012. 30

[43] P. Moireau, D. Chapelle, and P. Le Tallec. Joint state and parameter estimation for distributed mechanical systems. Comput Method Appl M, 197(6-8):659-677, 2008. 3

[44] P. Monk. Finite Element Methods for Maxwell's Equations. Oxford science publications, 2003. 45

[45] S. Nicaise and J. Valein. Stabilization of second order evolution equations with unbounded feedback with delay. ESAIM Control Optim. Calc. Var., 16(2):420-456, April 2010. 23

[46] A. Pazy. Semigroups of Linear Operators and Applications to Partial Differential Equations, volume 44 of Applied Mathematical Sciences. Springer-Verlag, New York, 1983. 18

[47] A. Preumont. Vibration Control of Active Structures, An Introduction. Kluwer Academic Publishers, 2nd edition, February 2002. 33

[48] J. Provost, C. Papadacci, J. E. Arango, M. Imbault, M. Fink, J.-L. Gennisson, M. Tanter, and M. Pernot. 3D ultrafast ultrasound imaging in vivo. Phys Med Biol, 59(19):L1-L13, September 2014. 2

[49] K. Ramdani, M. Tucsnak, and G. Weiss. Recovering the initial state of an infinite-dimensional system using observers. Automatica, 46(10):1616-1625, 2010. 3, 6, 7, 15

[50] P. Stefanov and G. Uhlmann. Thermoacoustic tomography with variable sound speed. Inverse Problems, 25(7):075011-17, June 2009. 8

[51] M. Tanter and M. Fink. Ultrafast imaging in biomedical ultrasound. 2014. 2

[52] M. Tucsnak and G. Weiss. Observation and control for operator semigroups. Birkhäuser Advanced Texts: Basler Lehrbücher. Birkhäuser Verlag, Basel, 2009. 24, 27, 45

[53] E. Zuazua. Propagation, Observation, and Control of Waves Approximated by Finite Difference Methods. SIAM Review, 47(2):197, 2005. 10, 30 